\title{
Herbal Immunomodulators - A Remedial Panacea for Designing and Developing Effective Drugs and Medicines: Current Scenario and Future Prospects
}

\author{
Ruchi Tiwari $^{* *}$, Shyma K Latheef ${ }^{2}$, Ishtiaq Ahmed ${ }^{3}$, Hafiz M. N. Iqbal ${ }^{4}$, Mohammed Hussen Bule ${ }^{5}$, \\ Kuldeep Dhama ${ }^{6}$, Hari Abdul Samad ${ }^{7}$, Kumaragurubaran Karthik ${ }^{8}$, Mahmoud Alagawany ${ }^{9}$, \\ Mohamed Ezzat Abd El-Hack', Mohd. Iqbal Yatoo ${ }^{10}$, Mayada Ragab Farag ${ }^{11}$
}

\author{
${ }^{1}$ Department of Veterinary Microbiology and Immunology, College of Veterinary Sciences, UP Pandit Deen Dayal \\ Upadhayay Pashu Chikitsa Vigyan Vishwavidyalay Evum Go-Anusandhan Sansthan (DUVASU), Mathura, Uttar \\ Pradesh, 281001, India \\ ${ }^{2}$ Immunology Section, ICAR-Indian Veterinary Research Institute, Izatnagar, Bareilly 243122, Uttar Pradesh, India \\ ${ }^{3}$ School of Medical Science, Gold Coast Campus, Griffith University, Southport QLD 4222, Australia \\ ${ }^{4}$ School of Engineering and Science, Tecnologico de Monterrey, Campus Monterrey, Ave. Eugenio Garza Sada \\ 2501, Monterrey, N. L., CP 64849, Mexico \\ ${ }^{5}$ Department of Pharmacy, Clloege of Medicine and Health Sciences, Ambo University, Ambo, Ethiopia \\ ${ }^{6}$ Division of Pathology, ICAR-Indian Veterinary Research Institute, Izatnagar, Bareilly 243122, Uttar Pradesh, \\ India \\ ${ }^{7}$ Division of Physiology and Climatology, ICAR-Indian Veterinary Research Institute, Izatnagar, Bareilly 243122 , \\ Uttar Pradesh, India \\ ${ }^{8}$ Central University Laboratory, Tamil Nadu Veterinary and Animal Sciences University, Madhavaram Milk \\ Colony, Chennai, Tamil Nadu - 600051, India \\ ${ }^{9}$ Department of Poultry, Faculty of Agriculture, Zagazig University, Zagazig, 44511, Egypt \\ ${ }^{10}$ Krishi Vigyan Kendra Nyoma, Sher-E-Kashmir University of Agricultural Sciences and Technology of Kashmir, \\ Shalimar, Srinagar 190025 Jammu and Kashmir, India \\ ${ }^{11}$ Department of Forensic Medicine and Toxicology, Veterinary Medicine, Zagazig University, 44511 Zagazig, \\ Egypt
}

*Correspondence: Ruchi Tiwari, Department of Veterinary Microbiology and Immunology, College of Veterinary Sciences, UP Pandit Deen Dayal Upadhayay Pashu Chikitsa Vigyan Vishwavidyalay Evum Go-Anusandhan Sansthan (DUVASU), Mathura, Uttar Pradesh, 281001, India; Tel. +91 9412566731; Fax: +91-565-2470819. Email: ruchi.vet@gmail.com

\section{Short running title \\ "Immunomodulatory herbs as effective drugs and medicines"}

\begin{abstract}
Constant exposure to various stressors, such as immune pressure, rapidly increasing population, deleterious changes in the ecosystem, climate change, infection with emerging and re-emerging pathogens, and fast-paced lifestyle, is a critical factor in the globally increasing incidences of immunocompromising health conditions, as well as stress. Synthetic chemotherapeutic agents, which are widely available in the commercial market, may be highly efficacious, but most are immunosuppressive and exert many side effects. Undoubtedly, the pivotal characteristics of immunostimulants and immunomodulators in the maintenance of the health and productivity of humans, as well as animals, cannot be overlooked. Numerous herbs used in ethnoveterinary medicine can be successfully employed as adjuvant rehabilitators to negate the deleterious effects of chemotherapeutics. The sources of these medicinal remedies are part of long traditions in different regions of the world, such as Indian Ayurveda and Traditional Chinese Medicine, which have been developed through empirical experience. Traditional medicine employs a holistic approach to the prevention of disease and traditional herbal medicines are a source of many components with high therapeutic value that are used in modern allopathic medicine. Globally, many studies have been conducted on these herbs and have revealed unique active constituents that activate the innate immune system through the stimulation of macrophages and lymphocytes, and modulation of the cytokine profile, which leads to a state of alertness with a subsequent reduction in the
\end{abstract}


incidence of infection. Immunomodulatory constituents with herbal origins are termed as phytochemicals, including flavonoids, glycosides, polysaccharides, terpenoids, essential oils, various bitters, and alkaloids; all these compounds exert vital, multidimensional effects. Efforts have focused on screening plant preparations to identify immune adjuvant properties; furthermore, several potent phytol adjuvants have been experimentally proven to downregulate inflammatory reactions in addition to enhance specific adaptive responses to vaccines. In this review, we discuss the current status and future prospects regarding the immunomodulatory potential of various herbs and plants and their promising utility for designing and developing effective drugs and medicines in safeguarding the health of humans, animals, and poultry.

Keywords: Herbs, plants, immunomodulation, drugs, medicine, health, therapy

\section{Introduction}

The relationship between animals and plants in nature is considered inseparable. Since the dawn of life on earth, they have coexisted and flourished in a symbiotic manner. Since the advent of human civilization, this relationship has been analyzed thoroughly and improved to a great extent. Ancient societies were closely associated with plants and animals, which were found in and around their living premises, as well as used for their daily necessities such as food, shelter, clothing, and medicines. Three hundred years ago, herbal therapy was recognized as holistic healing practice to counter physical ailments in Asia [1, 2]. The advent of modern biotechnology persuaded Western medical practitioners promptly to utilize Japanese Kampo (JK), Traditional Chinese Medicine (TCM), and Indian Ayurveda (IA) as healing therapeutics, the efficacy of which was strongly refuted in the $19^{\text {th }}$ and $20^{\text {th }}$ centuries. Many complex herbal mixtures used in traditional medicine require minimal processing, which has allowed the increasingly accepted integration of herbal medicines with customary treatment regimes, such as acupuncture, in the treatment of clinical disorders such as asthma, menstrual pain, headaches, immune problems, and various types of cancer [3-5]. Presently, the power of herbal therapy is being admired globally, and a wide array of studies and their results can be cited, to justify their successful impact in various treatments $[6,7]$.

The importance of herbal medication can be found in spiritual inscriptions from the Indian subcontinent, such as the Rigveda (10:97:2) and Atharvaveda (11:4:10), wherein numerous plants with the capacity to cure a variety of health problems have been described. The Rigveda also detailed the power of several herbal extracts to potentially penetrate into our muscles and joints as remedies for skeletal disorders (10:97:12). Ethnic concepts are passed orally between generations and accepted through empirical demonstrations. Traditional therapeutic approaches perceived by the public for animal care constitute the scope of ethnoveterinary medicine (EVM). Ethnoveterinary materia medica cover a broad range of practices, including appeals to spiritual forces, manipulative surgeries, and the use of plants/herbs, earth minerals, and animal products (fat, butter, and milk) [8].

The value of immunostimulants in augmenting and maintaining a healthy immune system is well recognized. A stimulated immune system could fight better for any impending infections, but it also exerts indispensable effects on stress- and infection-induced immunosuppression. The immune systems of higher vertebrates are very complex and consist of numerous immune cells and various cytokines; thus, it is difficult to achieve a specific and targeted level of immunostimulation [9]. Monovalent solitary immunomodulatory approaches are believed to be ineffective in maintaining adequate immunostimulation, and multiple immunomodulatory approaches may be necessary to achieve clinical success. One of the most promising alternatives to commonly used antibiotic treatments is the utilization of immunomodulators for the augmentation of defense responses in the subject of interest $[6,10]$. Several immunomodulators have been characterized, including substances isolated and purified from plants and microbes. A natural product or synthetic substance can act as an immunomodulator through the stimulation, suppression, or modulation of the innate or adaptive arms of the immune response [11-13]. The use of Rasayana drugs as immunomodulators can be an alternative to the usual chemotherapy for various ailments, particularly when immunoactivation or suppression is considered necessary, as in the case of autoimmune diseases. The idea that herbal antioxidants such as Shilajit and Chyavanprash Awaleha have concomitantly shown significant immunomodulatory activities resulted in the concept of using Rasayanas for health, which is gaining more support [14, 15]. Moreover, innate immunity, 
essentially the non-specific immunomodulation of granulocytes, macrophages, and NK cells, and their functions, has been upregulated via the use of medicinal plants [16-22].

Traditional herb-oriented therapeutic approaches have been employed particularly in tropical developing countries where these are widely accepted as effective treatments for the human [23]. At the outset, the orientation of herbal medicine is empirical and holistic, in contrast to a modern reductionist approach that seeks knowledge of the specific active constituents. The renewed appraisal and exploitation of traditional knowledge can significantly contribute to the identification of novel active components from herbs $[24,25]$. Currently, $50 \%$ of the modern synthetic medicines are derived from or based on phytochemicals $[26,27]$. The multiple actions of herbal preparations are claimed to have a lower risk of drug resistance, in addition to being more environmental friendly, compared to modern medicines. Herbal preparations constitute a large section of ethnoveterinary medicines and many of them were used to boost the immune status of domestic animals. In addition to their immunoregulatory activities, natural products have various beneficial effects, including antipyretic, antioxidative, anti-inflammatory, antiulcer, antidiabetic, cytoprotective, and anticancer effects, and exert health effects, such as the improvement of cervical spondylotic myelopathy [19, 28-34]. Herbal plants and their medicinal preparations have been identified as effective antimicrobial agents that could provide protection from the adverse health effects of infectious diseases, including the emergence of major global viral pathogens [19, 35-40]. For example, a "cytokine storm" was implicated in the death of patients during an influenza outbreak. Several herbal drugs have been studied to prevent this cytokine storm. Flavonoids, polyphenols, and triterpenoids were the active components in several plants that were reported to prevent cytokine storm during influenza [41].For a long time, botanical drugs have been considered crude owing to dilute amounts of many active principles. In contrast, many experiments have shown that plants derive their efficacy from the synergistic effects of several compounds [42-45]. Many researchers adhere to the theory that multicomponent remedies acting on multiple targets, which alter the complex equilibrium of cellular functions, are more favorable than drugs with a specific action [46-48].

Herbal extracts may confer potential advantages, including easy accessibility, low cost, the convenience of preparation, and usage in human medicine, animal health, and animal production [19, 4953]. In bioprospecting (drug discovery from nature), the utilization of traditional knowledge, such as systems like Ayurveda, is of great importance. Therefore, the utilizing traditional knowledge and the use of science and technology with a perspective on systems biology is imperative in this regard $[19,24,54$, 55]. The use of herbs and plants or their products has become of increasing interest in both the health care sector and scientific circles. Thus, there is a great need to change the focus towards such complementary and alternative systems in order to maintain an ample supply of real medicines $[19,35$, $56,57,59]$. The present review describes the role of immunomodulatory plants, herbs, and herbal preparations for the improved health of humans, animals, and poultry. This article will be useful for researchers in many fields, including veterinary science, medicine, pharmacy, agriculture, and the livestock and poultry industries.

\section{Ayurveda and Rasayana}

Ayurveda, developed in India, is the earliest medicinal system, established in the period circa 3147 BC [60]. Similar to the many other traditional practices, Ayurveda is supported by an enormous range of herbal and mineral medicines. Ayurveda follows four approaches to prevent diseases in human and animals: Parakratishapanum (maintenance of health), Roganasmani chikitsa (cure of disease), Naisthika chikitsa (spiritual therapy), and Rasayana chikitsa (herbal product therapy). Nakul Samhita, the oldest treatise, was written in 5000 B.C. and is another ancient Indian literature describing the treatment of animals with herbs [61]. Rasayana chikitsa, a section of the Ayurveda, uses plants believed to strengthen and promote the health of animals and human beings. Triphala, which has been recognized as a universal panacea in Indian Ayurvedic therapy for rejuvenating debilitated organs, is a unique Rasayana formulation derived from the active constituents of three valuable medicinal herbs: Terminalia chebula, T. bellirica, and Phyllanthus emblical Emblica officinalis [45]. Historically, Rasayana drugs with hepatoprotective and metabolism-enhancing properties have been included as a part of rejuvenating recipes [45, 62]. The effectiveness of Rasayana drugs that are still in practice for the treatment of various ailments has been strongly linked to their antioxidative properties, which antagonize oxidative stressors and free radicals, and their immunomodulatory effects [32, 63-65]. Many of the drugs regulate 
immunological and neuroendocrine systems [13, 66, 67]. The benefits of Indian herbs are well recognized, and their extracts are being analyzed for potential medical uses [2, 19, 68, 69]. There is a keen interest in the systematical screening of plant extracts of different countries worldwide for their curative properties [70-73].

\section{Herbs as Immunostimulators}

Microbes are everywhere; with their warmth and rich supply of nutrients, animal bodies are an excellent place for them to perpetuate. Survival in these hostile conditions emphasizes the significance of the immune system. Animals are endowed with elaborate and powerful immune mechanisms. Weakened immunity may be the cause for many of the illnesses. In this context, the significance of non-specific immunostimulants is well recognized. The endogenous potential of the immune system can be stimulated with immune enhancers, thereby creating a state of alertness to microbial invasion and subsequently reducing the chances of infections [74, 75]. Herbal preparations modulate immune functions by immunostimulation, immunosuppression, or immunoregulation (Fig. 1). Further, they can increase the efficacy of chemotherapy for the control and prevention of infections. Three classes of nonspecific immune enhancers are well recognized; those derived from microbial products, synthetic chemical compounds, and herbal extracts. Modified microbial products and synthetic compounds are constantly refined as immune adjuvants [76-78]. The use of herbal preparations can also be an effective method to boost the immune system. Early systematic studies identified a variety of herbal compounds as immunostimulants [79], with proven stimulatory effects on macrophages, T and B lymphocytes, and the modulation of cytokine secretion, immunoglobulin secretion, and class switching [29, 80-82].

In the classical examples of immunomodulators, many plant extracts can act as botanical adjuvants for conjugation with vaccines, with high level of usefulness in human and animal medicine. The extracts of Tinospora cordifolia have been used as an immunomodulator, along with chloroquine, for the treatment of hyper-reactive malarial splenomegaly with promising results [83]. Based on their previous experience, Ragupathi et al. [84] screened seven extensively used herbal extracts to determine their potential as cancer vaccine adjuvants in mice. Their results confirmed the strong immunostimulatory activity of Coriolus extracts, 95\% ethanol extract of Astragalus, yeast-1 glucan, and Maitake extract. Astragalus polysaccharides in sow diet were shown to improve the levels of growth factors EGF and IGF-1 in colostrum post-parturition [85]. A major aim for this research is the development of an effective herbal formula that can provide synergistic beneficial effects without any potential adverse reactions. The vast amount of literature indicates the medicinal uses of traditional and modern day herbs, but comparatively few herbal preparations are studied by using structured experiments. Herbal preparations have displayed a range of immunomodulatory activities both in vitro and in vivo; and some are awaiting validation for clinical use [75, 86-88]. 


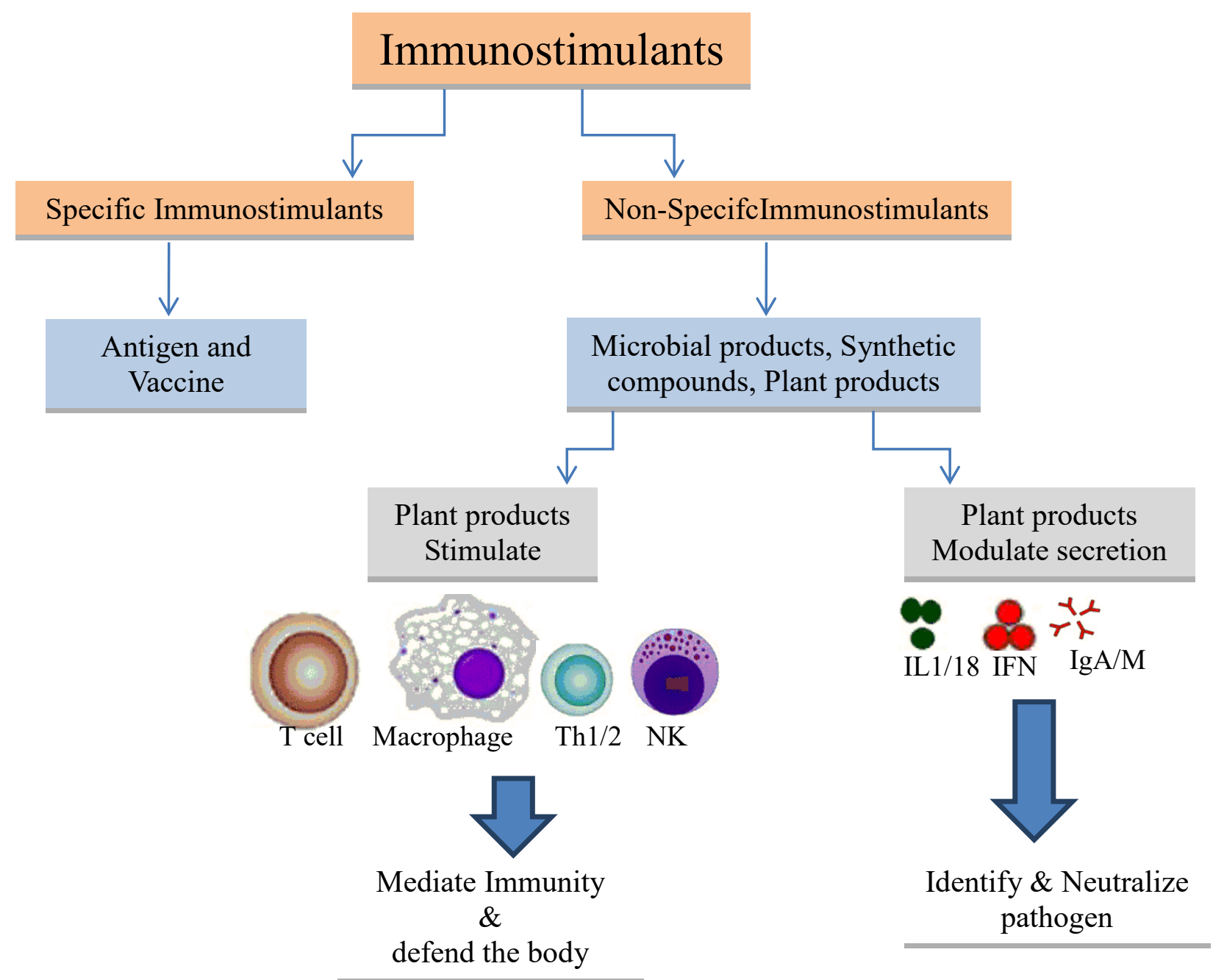

Figure 1: The immunostimulant and immunomodulatory activity of herbs

\section{Plants and Herbs with Immunomodulatory Properties and Applications}

\section{Glycyrrhiza glabra (Licorice or Sweetwoods)}

Glycyrrhiza glabra is native to Central Asia and the Mediterranean region. The roots are commonly known as licorice or sweetwoods. The root extract of this plant has been used historically in Egyptian, Chinese, Indian, and Greek medicine as carminative and expectorant. Similarly, it is described in Ayurveda as Rasayana against throat infections [89]. The major components of licorice are triterpene saponins, flavonoids, and pectins, which are responsible for its pharmacological activity [90]. Glycyrrhizin (GL), contains a triterpene compound responsible for the sweetness of its root; the flavonoid compound, isoliquiritin, is responsible for the yellow color of licorice [91]. Licorice contains compounds that display steroid-like anti-inflammatory activity by the inhibition of phospholipase A2 and they interfere with platelet aggregation [92]. Glycyrrhiza plant extract was reported to increase resistance to Candida albicans and the herpes simplex virus through its influence on the secretion of Th- 2 cytokines [93]. GL was found to lower lipid peroxidation in animal models [94].

The anti-inflammatory and hepatoprotective function of licorice reportedly increased the production of NF- $\mathrm{KB}$ and IL-10 by GL [95]. The Bcl-2/Bax family of genes, which are the regulatory factors for apoptosis, were found to be modulated by the components of GL root, and can be considered in elucidation of its reported cytoprotective action. Licorice contains two chemical components, isoliquiritigenin and naringenin, which have been reported to enhance regulatory $\mathrm{T}$ cells and therefore may be cause of the anti-inflammatory properties and efficacy against autoimmune diseases [96]. Dorhoi et al. [97] induced enhanced cellular immunity in birds treated with the ethanol extracts of licorice. The 
purified saponins from this plant used in ISCOM preparation significantly enhanced the immune response of broiler chickens against Eimeria tenella [98]. Therefore, Chinese licorice (Gan-Cao) was assessed for its active components and indication of its therapeutic value [99]. G. uralensis (Chinese licorice) was reported to regulate the cytokine IL-7, which is involved in immune cell proliferation and maturation, and can thus act as anticancer agent [100]. Licorice root powder, capsules, or slices are available for purchase and known for their multiple applications. According to Jessica Houdret [101], it is used for stomach disorders, sore throats, snake bites, scorpion bites, and food poisoning. Glycyrrhizic acid from Glycyrrhiza glabra demonstrated inhibitory properties against Leishmania donovani in an in vitro study, which may be attributable to the depletion of ergosterol in promastigotes [102].

The root of Glycyrrhiza glabra possesses multiple properties; it can be used as a sweet refrigerant, aphrodisiac, alexipharmic, alterant, emetic, diuretic, demulcent, expectorant, emmenagogue, and intellect promoting. Furthermore, successful use in bronchitis, cough, cephalalgia, fever gastralgia, gastric ulcers, hyperdipsia, ophthalmopathy, pharyngodynia, skin diseases, cuts, and wounds has been demonstrated $[103,104]$. Recently, several other bioactive chemicals have been isolated from the plant, including isoprenylated phenolics and echinatin, which confers the hepatoprotective properties [105]. Carbenoxolone (18- $\beta$ glycerrhetinic acid and hydrogen succinate), an analog of glycerrhetinic acid, is used in the treatment of certain alimentary tract ulcerative conditions, such as peptic ulcer (Fig. 2). Moreover, it has been indicated that acute pretreatment of adrenalectomized male rats with carbenoxolone sodium, the water soluble succinate derivative of the glycyrrhetinic acid, caused both cortisol and corticosterone to display significant mineralocorticoid-like activity, particularly $\mathrm{Na}^{+}$ retention [106]. Various studies have indicated the application of G. glabra as a strong medicine for the treatment of inflammatory disorders and immunosuppressive conditions, in which it can exert soothing action against different ailments especially affecting respiratory and digestive systems. An overview on the immunomodulatory effects of G. glabra is presented Fig. 3.

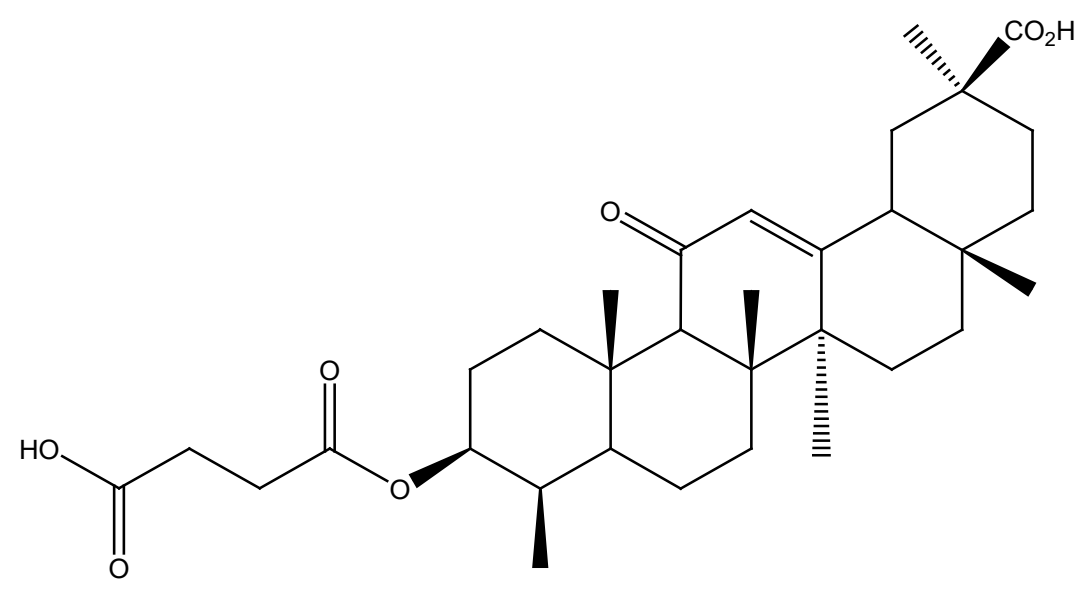

Figure 2: Chemical Structure of Carbenoxolone 


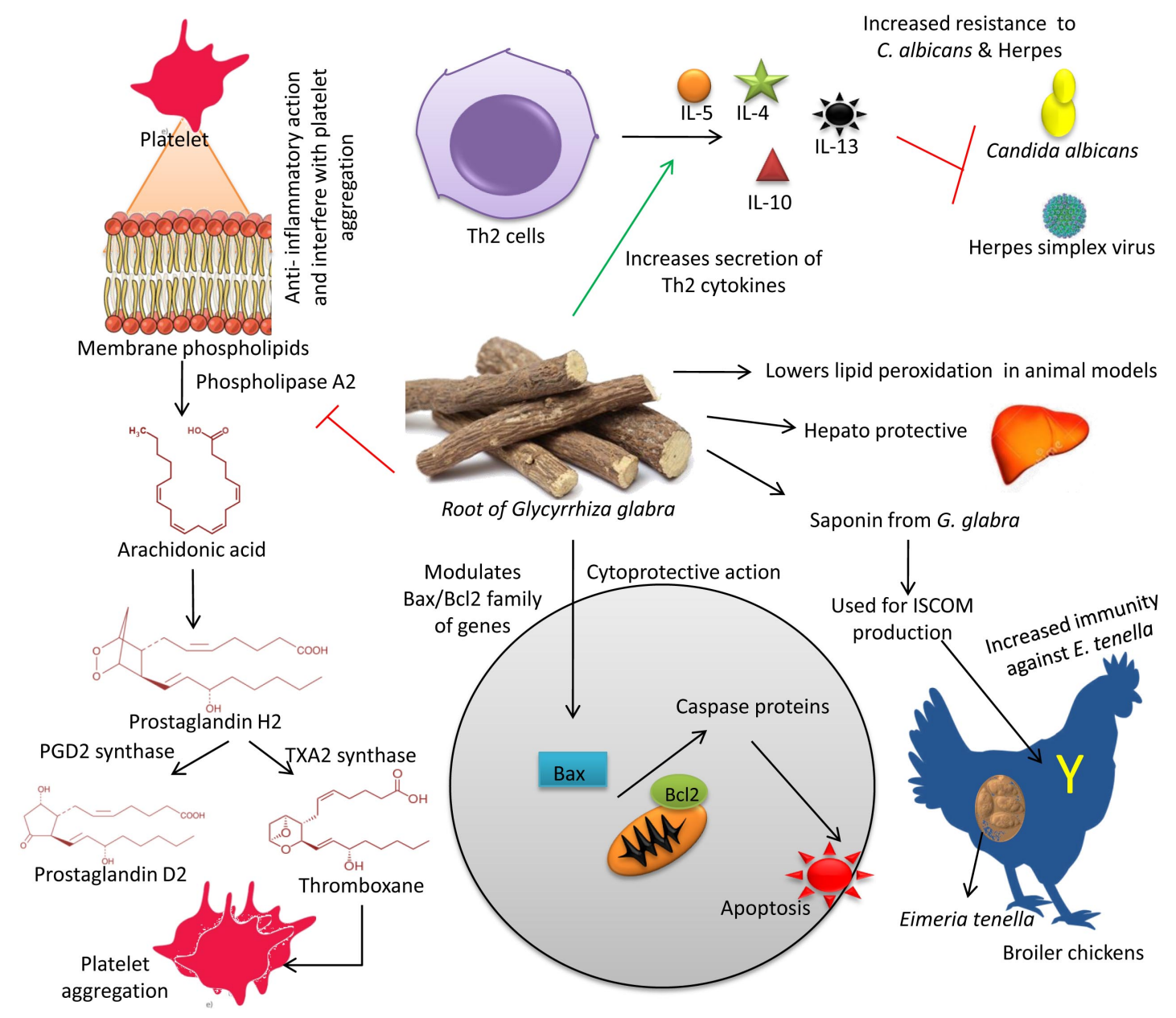

Figure 3. An overview on the immunomodulatory effects of Glycyrrhiza glabra

\section{Withania somnifera ('Ashwagandha' - Solanaceae)}

Ashwagandha is an Indian ginseng, which is an important Rasayana in Ayurveda. In traditional and Ayurvedic medicine, Ashwagandha has been used for approximately 3000 years [107]. It is well known as a cytoprotective, immunomodulatory, antibacterial, antioxidant, and antitumor agent [108, 109]. The active constituents, commonly called as withanaloids, are made up of steroidal lactones and alkaloids (Fig. 4), among which the most biologically active constituents are withaferin A and withanolide A, with the bioavailability of withanolide being 1.44 times more than that of withaferin A [110]. Various pharmacological and metabolism based studies have been conducted in different species [111], including buffalo [112] and rabbit [113], which indicated that the peak plasma concentration was attained at 1-2 $\mathrm{h}$ after oral supplementation, with a biological half-life in the range from 18.29-27.69 h [33]. Extensive research is in progress on the pharmacological aspects of Withania, which suggested the application of these withanaloids as promising drug candidates in oncotherapy, as well as in neurological conditions $[33,110]$.

The extracts of Withania somnifera are proven diuretic, anti-inflammatory, antitussive, anticancerous, sedative/anxiolytic, and antioxidant agents [114-116]. Ashwagandha extracts enhanced the Nitric Oxide (NO) synthase activity of macrophages and increasing their microbe killing power [117]. The anticancer value of the plant was well supported in some studies. It modulated the effects of cytotoxic lymphocytes and led to reduced tumor growth. In one of the experiments, it was indicated that its action against the growth of breast and colon cancer cells was better than that of doxorubicin [118]. Enhanced NK cell activity reduced the tumor growth in mice [119]. Winters [120] recently reviewed the potential use of ashwagandha extracts in cancer chemotherapy and found that Withania extracts significantly increase the cell-mediated immunity in normal mice [119]. Similar immune enhancements were also found in mice with myelosuppression induced by cyclophosphamide, azathioprin, and 
prednisolone [121]. Castleman [107] reported that several Indian animal studies proved a stimulatory effect on the immune system, enhanced antibody and red blood cells levels, and an increased number of white blood cells to devour germs. Moreover, the same author reported another Indian study on laboratory animals, which recorded reduced stress following the administration of Ashwagandha in one group, before the administration of electrical shocks that produced chronic stress. A study reported that administration of $W$. somnifera extract to dexamethasone-induced immunocompromised mice showed a marked increase in primary and secondary antibodies and also cell-mediated immune response. Thus, the study proved that $W$. somnifera extracts possessed immunostimulatory properties [122]. W. somnifera administration in mice with cyclophosphamide-induced toxicity resulted in an increase in WBC, platelet, and lymphocyte counts [123].

The root extract of $W$. somnifera was been found to elicit a cell-mediated immune response in BALB/c mice, which was mediated by Th1-stimulating cytokines [124]. The alcoholic root extract of $W$. somnifera showed potential immunomodulatory and antibacterial properties in guinea pigs experimentally infected with $E$. coli and improved hematological, biochemical, and immunological parameters by suppressing the levels of the inflammatory cytokine TNF- $\alpha$ [125]. The anxiolytic, antiinflammatory, and anti-apoptotic properties of aqueous extract of $W$. somnifera (WS) leaves have been scientifically validated by studies performed in sleep-deprived rats. The stress owing to sleep deprivation was ameliorated by WS extract, as measured by the modulation of immune response markers, such as GFAP, TNF- $\alpha$, IL-6, OX-18, and OX-42. The expressed levels of NF- $\kappa B$, AP-1, Bcl-xL, and cytochrome c confirmed the reduction in stress-induced apoptosis and supported the inclusion of WS as dietary supplement [126].

Ashwagandha forms the principal component of many polyherbal preparations (Immu-21, Amrit $^{R}$, Su-Ruksh ${ }^{\mathrm{R}}$, Ashwgandha ${ }^{\mathrm{R}}$, and ImmuPlus $^{\mathrm{R}}$ ). Immu-Plus ${ }^{\mathrm{R}}$ was shown to increase the antibody titer in dogs in addition to the stimulation of the blastogenic capacity of $\mathrm{T}$ and $\mathrm{B}$ cells. It has been frequently examined for immunopotentiation along with vaccines. In pups, it has shown better immune stimulation when used together with vaccination against viral diseases such as canine parvoviral infection and rabies [127]. Similar encouraging results have been observed with infectious bursal disease and Newcastle disease vaccines of poultry $[128,129]$. Another polyherbal preparation, Immu- $21^{\mathrm{R}}$, has shown immunomodulatory effects leading to modest improvements in the condition of HIV patients [130]. Experimental evidence attributed multiple actions to this herb including anti-inflammatory, analgesic, and anti-stress effects, along with better anabolic activities [131-133]. Ashwagandha is known for its excellent immunostimulating potential among herbs, and it is one of the most extensively studied and widely used herbal immunomodulators in a variety of species. In silico studies involving different databases, such as DPED, UNPD, PubChem, Binding DB, ChEMBL, KEGG, and STRING, have helped to identify immune system pathways and target proteins/withanolide-phytosterols responsible for immunomodulation, and facilitated the understanding of the molecular mechanism of immunomodulation exerted by Withania somnifera [134]. An illustration on the salient immunomodulatory effects of $W$. somnifera is presented in Fig. 5.

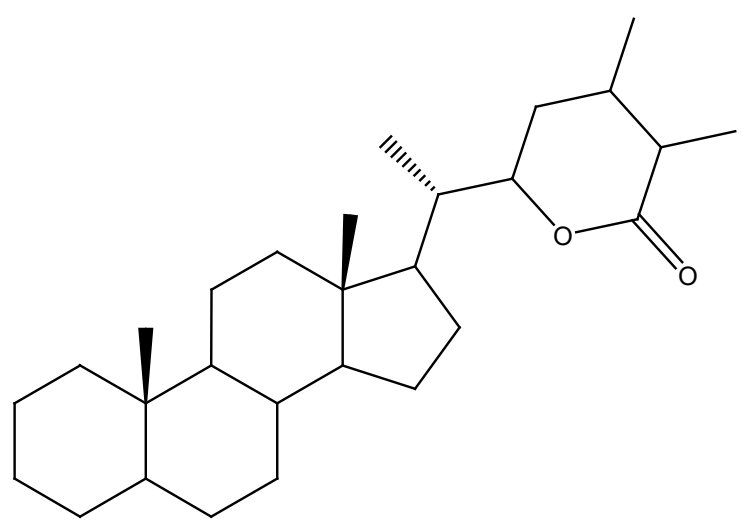

Figure 4: The basic structure of withanaloids; C-28 steroidal lactones mainly localize in leaves. 


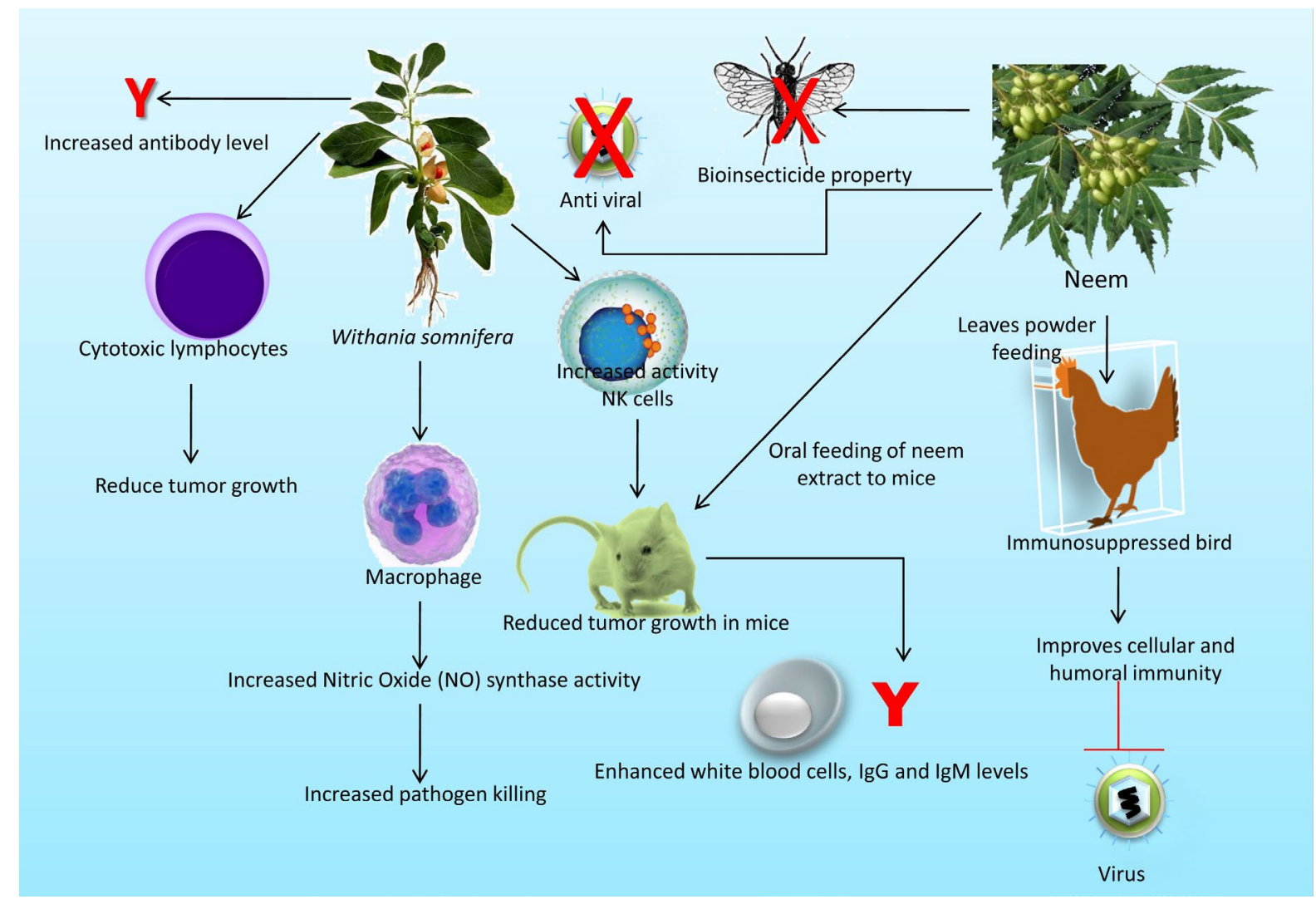

Figure 5. Immunomodulatory effects of Withania somnifera and Azadirachta indica

\section{Azadirachta indica ('Neem' - Meliaceae):}

Neem is a native of southeastern Asia and is widely spread throughout India. It is highly esteemed tree with several beneficial properties and applications $[135,136]$. Almost all parts of this plant are bitter in taste and utilized in traditional remedies. The bark of the plant has been found to exert biological effects, such as astringent, insecticidal, liver tonic, anthelmintic, and expectorant properties. It can also be useful in treating leprosy, eczema, leucoderma, skin diseases, diabetes, amenorrhea, hemorrhoids, bronchitis, lumbago, malaria fevers, otalgia, syphilis, and burning sensations [137]. The leaves were also proven to have the abovementioned properties of bark, in addition to potential used to treat tuberculosis, boils, and ophthalmopathy $[138,139]$. The flowers of neem possess anthelmintic, ophthalmic, stomachic, and tonic action against general debility. The seeds are also proven to have these properties along with some specific effects like thermogenic, purgative, uterine stimulant, and urinary astringent effects. The seeds also can be used to treat tumors, leprosy, odontalgia, hemorrhoids, pulmonary tuberculosis, ophthalmopathy, antenatal disease, wounds, ulcers, diabetes, and otorrhea [140]. The neem oil is as effective as other plant parts in ameliorating these conditions especially against chronic skin diseases, syphilitic sores, ringworm, malaria fever, scabies, indolent ulcers, and leprosy [141, 142].

Natural healers have used neem plant preparations for the treatment of some diseases, such as leprosy, GIT related ailments, seizure, malaria, and ulcers [143]. Furthermore, it is found to enhance the mitogenic activity of Con-A to splenocytes and also effective against allergic disorders by desensitizing the host specific allergens [144]. Plant contents are especially known for bioinsecticide property, where they were found to be effective against many insect species of veterinary and medical importance, nearly 400 insect species, including spiders and nematodes [145]. In the first experimental study in a mouse model, neem oil showed increased activity of macrophages with higher expression of MHC II, IFN- $\gamma$ production, and enhanced lymphocyte proliferation activity [146]. Neem leaf extract given orally in mice produced effects, such as the enhancement of white blood cells, IgG, and IgM levels [147]. Powdered neem leaves were used in immunosuppressed birds that augmented both humoral and cell-mediated 
immunity, which prevented further infection with viruses [148]. Neem leaf extracts were also found to have adjuvant activity in the enhancement of the immune response against poor antigen vaccines [149]. Several reports have revealed the value of neem extracts for their antiviral and anticancerous properties [150-153]. Neem possesses phytochemicals including tannins, salicylic acid, and gallocatechin, which are especially prevalent in their bark, and these constituents are reported to be the anti-inflammatory principles [154]. Pathological evaluations based on the toxicity studies revealed the testicle, lungs, liver, and kidneys were the major vital organs affected by neem oil overdosage [137, 155]. The salient immunomodulatory effects of $A$. indica are depicted in Fig. 5.

\section{Tinospora cordifolia (Giloy- Menispermaceae):}

Tinospora cordifolia (TC) is a famous herb with many local names (Guduchi, Amrita, Giloy) and is known for boosting the immune system by immunomodulatory and cytoprotective activities through various non-specific immune mechanisms [156]. It is mainly found in India, Sri Lanka, and Myanmar [157]. The effective principles of this plant have been extensively studied and include wide-ranging chemical groups, such as alkaloids, diterpenoids, flavonoids, 11-hydroxymustakone, $N$-methyl-2pyrrolidone, $N$-formylannonain, cordifolioside A, magnoflorine, tinocordiside, syringin, and lignins [156, 158]. Many of these principles have potent immunostimulatory properties, along with several other properties similar to the stem, with gastric effects including astringent, anodyne, appetizer, alterant, antiperiodic, anthelmintic, antiemetic, carminative, galacto-purifier and tonic. In addition, antipyretic, anti-inflammatory, aphrodisiac, cardiotonic, depurative, expectorant, haematinic, and rejuvenating properties have also been reported [159]. General conditions, such as anemia, asthma, chronic and intermittent fevers, cardiac debility, erysipelas, dyspepsia, hyperdipsia, gout, jaundice, seminal weakness, splenopathy, and uropathy were also alleviated by this multi-faceted herb [27]. The alcoholic extract of Tinospora has been shown to possess greater immunomodulatory function through augmentation of antibody production [160], whereas the methanolic extract was excellent in the exhibition of antimicrobial properties, as evidenced by different in vivo studies [161, 162]. The treatment of the condition of visceral Leishmaniasis in a murine model with TC, along with cisplatin, was found to be effective as it reduced the undesirable effects of cisplatin and modulated the immune response from a Th2 to a Th1 type [163].

Preparations have shown that the activation of macrophages led to an increase in granulocytemacrophage colony-stimulating factor (GM-CSF), leukocytosis, and improved neutrophil function. TC was also reported to inhibit C3-convertase of the classical complement pathway. In one of the studies, polysaccharides ( $\alpha$-D-glucan) from this plant showed activation of NK cells, the complement system, and $\mathrm{Th}_{1}$-pathway cytokines, coupled with low nitric oxide synthesis $[164,165]$. The antitumor activity of TC was evaluated in cultured HeLa cells and it was revealed that the effect of the extract was comparable with doxorubicin treatment [166]. Leaf extracts of this plant protected finfish from experimental infection with Aeromonas hydrophila, which indicated its value as an immunoprophylactic agent [167]. A clinical study also linked the immunomodulatory role of the TC plant in the improved healing of diabetic foot ulcers and wounds in Wistar rats, by increasing the rate of epithelialization, and enhanced the scavenging of free radicals [168, 169]. Mukherjee et al. [170] studied Tinospora root extracts in the treatment of bovine clinical mastitis and observed the antibacterial and immunomodulatory effects. Tinospora plant components demonstrated an improved therapeutic ability in various maladies, from a throat infection $\left(\operatorname{Septilin}^{\mathrm{R}}\right)$, to rheumatoid arthritis (Rumalaya ${ }^{\mathrm{R}}$ ). The novel polysaccharide, $(1,4)-\alpha-\mathrm{D}$-glucan, was recently isolated from $T$. cordifolia. It was found to act as a TLR4 agonist, which indicated the potential use as an adjuvant $[171,172]$. T. cordifolia growing on neem was reported to possess better immunomodulatory properties than in the absence of neem [173]. Additionally, alcoholic extract of $T$. cordifolia is very useful in treatment and correction of knee osteoarthritis through the enhancement of the collagen deposition, mineralization of bones, increased production of osteocalcin, elevated expression of osteogenic gene, and increased the growth and differentiation of osteoblastic lineage cells [174]. Epoxy clerodane diterpene (ECD) from $T$. cordifolia induced apoptosis in cancer cells by genetically regulating the expression of the genes Cdkn2A, p53, and mdm2, and acted as effective and safe herbal anticancerous agent by the modulation of the genetic expresions [175]. An overview on the medicinal uses of T. cardifolia is shown in Fig. 6. 


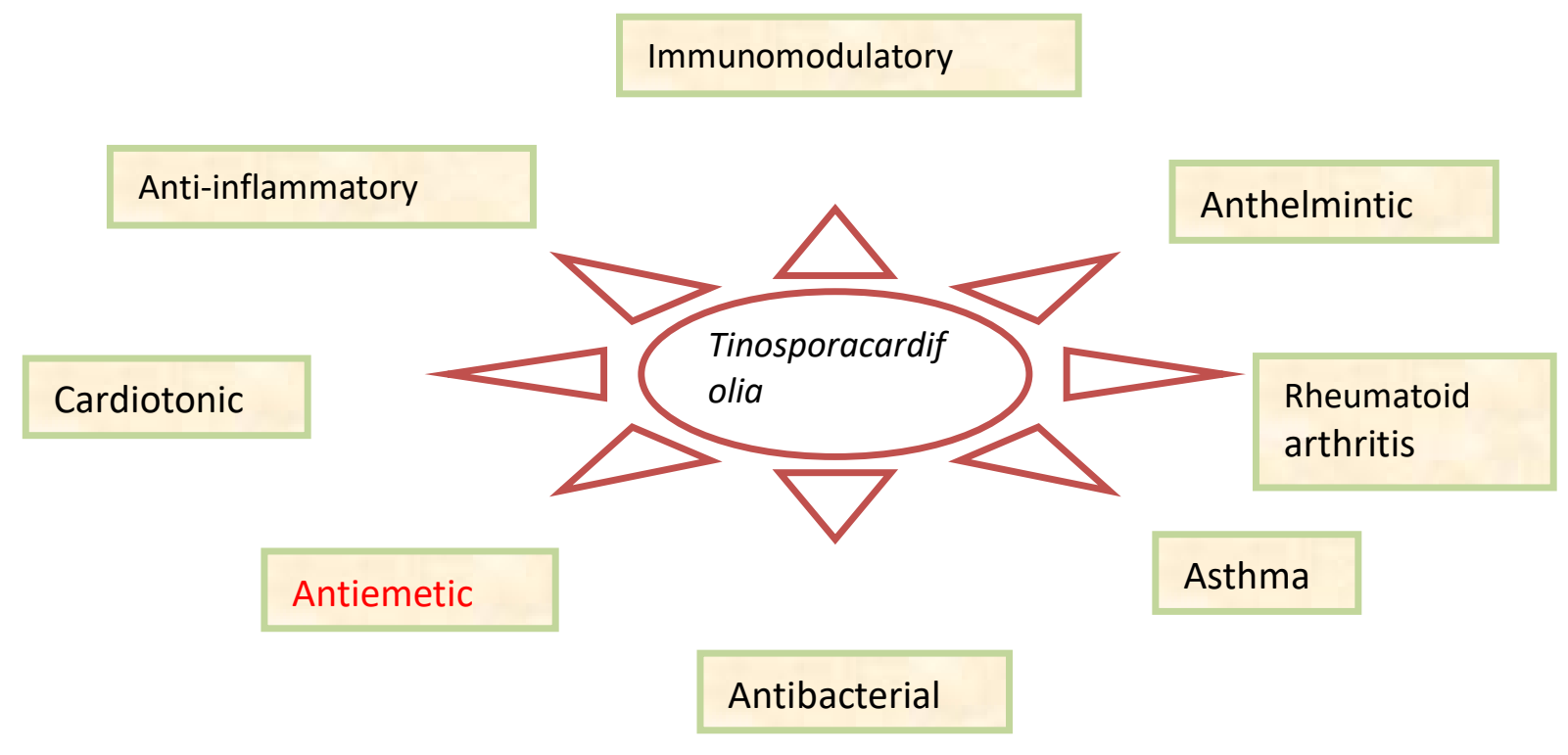

Figure 6: Medicinal uses of Tinospora cardifolia

\section{Echinacea spp.}

Echinacea is one of the most traditionally trusted herbs for a variety of purposes, such as cough, sore throat, and even insect bites. Before the emergence of antibiotics, this plant was extensively used in the treatment of infectious cases [176]. The value of Echinacea in reducing the duration and incidence of cold was recently shown by the analysis of multiple clinical studies [177]. There are three species of Echinacea plant Echinacea angustifolia, Echinacea purpurea and Echinacea pallida, which are of medicinal interest. E. purpurea is special for its reported immunomodulatory effects; this is clearly described in many reviews [178-180]. The immune boosting effects of this plant have also been recorded in many studies [181-184]. The active principles, polysaccharides, of Echinacea enhanced the nonspecific cytotoxic effects of macrophages and natural killer (NK) cells [107]. In vivo randomized studies demonstrated improved immuno-enhancing properties of Echinacea preparations, in particular to white blood cells production, thereby conferring antiviral, antifungal, and antibacterial effects to the plant [185, 186]. Further root extracts were found to be more efficient immune enhancers than those of leaf extracts [187]. The DNA microarray-based study of root and leaf-stem extracts of Echinacea revealed the different effect of both stimulatory and inhibitory effects on dendritic cell gene expression, which indicated a modulatory effect [188]. Sullivan et al. [189] reported the activation and production of IL-6, TNF, IL-12, and nitric oxides from macrophages in mice treated with Echinacea extracts. A standardized extract of Echinacea (Polinacea) also showed the similar modulatory effect on sheep neutrophils [190]. Recently, both root and leaf extracts effects on dendritic cells of mice were studied, and it was revealed that the observed immunomodulation depended on the portion of plant and extraction method [191]. The immunomodulatory-mediated amelioration of upper respiratory tract infections was reported after the supplementation of Echinacea plants/extracts via cytokine profile modification [184]. Echinacea purpurea extracts (EE) showed immunomodulatory potential and assisted in the development and maturation of dendritic cells by altering the activation of JNK, p38-MAPK and NF- $\mathrm{KB}$ pathways [192]. The overall evaluation of Echinacea indicated its application as a primary remedy for common infections, particularly those affecting the respiratory system. Currently, researchers are awaiting the further elucidation of its alternative therapeutic potential and usage as potent immunomodulatory herb.

\section{Uncaria tomentosa (Cat's claw)}

Uncaria tomentosa, an Amazonian vine, is another herb historically known for its efficacy in immunological and digestive disorders [193]. The inner bark and roots are highly valued for their medicinal properties in phytotherapy. There are different groups of phytochemicals in Cat's claw, including oxindole alkaloids, quinovic acid, glycosides, antioxidant chemicals (e.g., tannins, catechins, 
and procyanidins), plant sterols (e.g., beta-sitosterol, stigmasterol, and campesterol), and carboxyl alkyl esters (Fig. 7). In addition, Uncaria tomentosa possesses an array of other chemicals, including glycosides (e.g., pteropodine, mitraphylline, isometraphylline, and quinovic acid), sterols (e.g. campesterol, sitosterol, and stigmasterol), fatty acids (e.g., loganic acid, oleanolic acid, and palmitoleic acid), uncarine (A through F), and vaccenic acid (omega-7 unsaturated fatty acid) [141, 194]. Mitraphylline has been revealed to be the active principle in the suppression of inflammatory reactions and the regulation of activated immune cells [195]. Pharmacodynamic studies have shown that mitraphylline extracted from the bark exerted antiproliferative effect on cells, as evidenced by the growth inhibitory effects on glioma cells and neuroblastoma cell lines [196, 197].

The increased production of IL-1, IL- 6 , and IFN- $\gamma$ suggesting that immunoregulatory effect was reported in aqueous extracts and a mixture of oxindole alkaloids [198]. The extracts displayed enhanced lymphocyte activity owing to adjuvant immune activity observed by the pneumococcal vaccine: neutrophil ratio and persistent antibody titer responses towards 12 pneumococcal serotypes [199]. These plant extracts were tested in vitro, in vivo, and in gene expression studies displayed similar antiinflammatory activities, such as the inhibition of the activation of NF- $\kappa B$ and suppresses TNF- $\alpha$ synthesis [200]. The reduced production of TNF- $\alpha$ and scavenging of free radicals explained the cytoprotective function of Cat's claw [201, 202]. The aqueous extract of this plant was indicated in rheumatoid arthritis treatment [203]. The extracts could protect from lethal infections of Listeria monocytogenes and could significantly increase granulocyte-macrophage progenitor cells in bone marrow [198]. A 2\% gel of Cat's claw demonstrated antibacterial properties against Enterococcus faecalis in an in vitro study [204]. The plant extracts demonstrated both antiviral and cytokine modulation in human monocytes infected with dengue virus [205]. Cat's claw is useful for improving the symptoms of Alzheimer's disease by its antioxidant effects and also by peripheral blood vessel dilation in the brain by rynchophylline alkaloids $[206,207]$. It was reported that the immune-stimulating alkaloids pteropodine and isopteropodine have positive modulatory effects on brain neurotransmitters (5-hydroxy tryptamine) receptors; these are important drug targets in treating a range of ailments such as depression, anxiety, and obesity [208-210].

$U$. tomentosa contains pentacyclic as well as tetracyclic alkaloids, in particular of indole and oxindole, which impart two different chemotypes. As both types of alkaloids impart an antagonistic effect on each other, the medicinal application of these two alkaloid mixtures (pentacyclic and tetracyclic) has been reported as unsuitable [211]. The standardized preparation of Uncaria with a high proportion of pentacyclic alkaloids is recommended and used for therapeutic purposes [212]. Commercially available preparations, including a decoction, fluid extract, or capsules, are commonly used. For effective immune benefits, Cat's claw is prescribed at the rate of $1 \mathrm{~g}$ of powder in tablets or capsules for daily for human consumption to a maximum dose of $20 \mathrm{~g}$ or $2-3 \mathrm{~g}$, two to three times daily. The standard vine bark decoction for general health was $1 / 2$ to 1 cup, 3 times daily (http://www.biotecharticles.com).

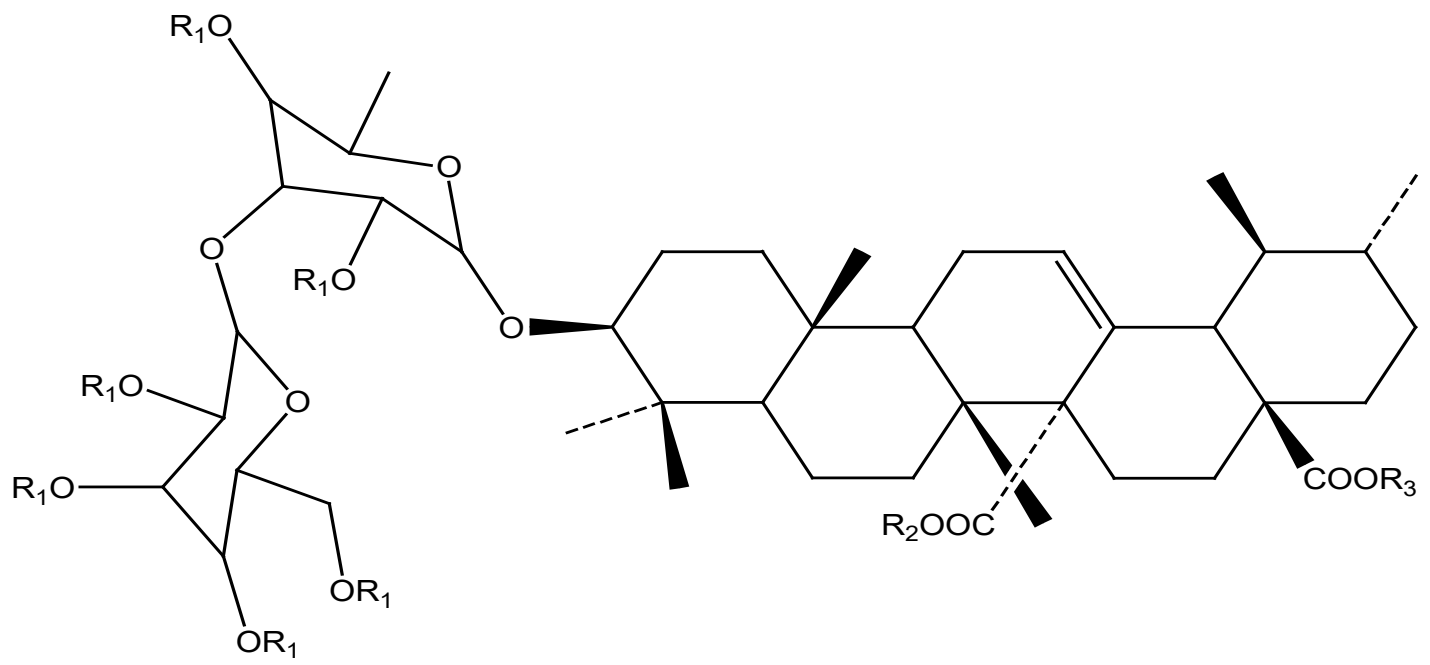

Figure 7: General structure for quinovic acid glycosides extracted from Uncaria tomentosa 


\section{Ocimum sanctum (Tulsi - Labiatae):}

With the local name of 'Tulsi,' Ocimum sanctumis was mostly regarded as a holy plant in Indian homes. Almost every part of this unique plant is believed to have medicinal properties and it has been traditionally used for analgesic, anticancer, and antistress effects [213-215]. The main parts of the plant commonly used are the leaves, flowers, stem, and seeds. The preparations from the plant are believed to reduce the period of illness and clinical symptoms in viral hepatitis patients [216]. Ursolic acid, rosmarinic acid, eugenol (Fig. 8), and carvacrol are the pharmacologically active components the plant; thus it can be assessed that the different pharmacological effects of tulsi in its natural form would result from the synergistic interactions of its various active phytochemicals [217]. The herbal preparations of this plant, with eugenol as active compound, possessed membrane-stabilizing properties on erythrocytes and mast cells, suggesting a possible use in allergic and inflammatory disorders [218, 219]. The immunostimulatory property of Eugenol is helpful in the treatment of immunosuppressed conditions [220]. The aqueous extract of leaves has the property of increasing the colony forming units in the spleen of mice that were thus protected from irradiation and infection [221]. Immunomodulatory properties were found to be maximum from the steam distilled extract of $O$. sanctum through humoral immunity, as evidenced by the in vivo studies in albino rats. Oil extracted from its seeds modulated both the humoral and cellular components of immunity, in particular through the GABAergic pathways [222, 223]. Ocimum sanctum essential oil was found to trigger apoptotic activity in breast cancer cells [224].

Extensive pharmacological studies have been conducted to evaluate the juice extracted from the leaves of $O$. sanctum and found have uncovered promising actions such as antiperiodontic, diaphoretic, expectorant, earache-relieving, and amelioration of gastric and hepatic disorders and skin diseases [87]. The slender roots were used as an antidote for snake bites and scorpion stings and decotions of the roots are used for the treatment of malaria, and as a diaphoretic. The plant is acrid, aromatic, and bitter. It is used as and alexeteric, demulcent, diaphoretic, digestive, diuretic, expectorant, febrifuge, stomachic, and vermifuge. It is also used for asthma, bronchitis, catarrhal fever, cardiopathy, gastropathy, genitourinary disorders, haematopathy, hepatopathy, hiccough, leucoderma, lumbago, otalgia, ophthalmia, ringworm, skin diseases, verminosis, and vomiting. The fixed oil of this plant was found to have potential antiinflammatory effects. Increased anti-sheep erythrocyte antibody response was also observed following the use of acetone extracts of the ocimum plant [225]. The aqueous extracts of this plant have shown enhanced antibody production along with neutrophil stimulation in fishes [226]. Mediratta et al. [227] reported an improvement cell-mediated and humoral immunity parameters in both stressed and nonstressed rats after the use of ocimum seed oil. The aqueous extract of Ocimum sanctum leaf infused via the intramammary route was found to increase neutrophil and lymphocyte count and increased phagocytic index in bovine mammary gland [222]. Aqueous and alcohol extracts of this plant stimulated the innate immunity of goldfish and protected them from common infections [228]. The extracts obtained from Ocimum sanctum were observed to be equally effective against the pathogenic gram negative and gram positive bacteria [229]. The counts of RBC and WBC, as well as the levels of blood protein and globulin, were increased by Ocimum sanctum extracts at levels of $2.5 \%$ and $5 \%$ in 15 - and 30 -day treatments in fish.

The potential of $O$. sanctum in the mediation of all these important effects may be attributed to the presence of bioactive and phenolic molecules such as saponin, tannins, steroid, flavonoids, eugenol, terpenoids, cardiac glycerides, and caryophyllene [230]. O. sanctum aqueous extract can exert antiulcerative properties, which are mediated through the induction of nitric oxide, and confirmed its ethnopharmacological potential in soothing peptic ulcers [87]. The leaves of $O$. sanctum contain volatile oil $(0.7 \%)$, of which $70 \%$ is of eugenol and $20 \%$ is of methyl eugenol; hence, the extract of Ocimum sanctum leaf improved immunity when incorporated into the diets at the levels of $0.0 \%, 0.05 \%, 0.1 \%$, $0.2 \%, 0.5 \%$ and $1 \%$ [231]. Although various species are present in Ocimum genus with different phytochemical composition, it is the high eugenol content that is responsible for the multiple pharmacological effects of $O$. sanctum. 


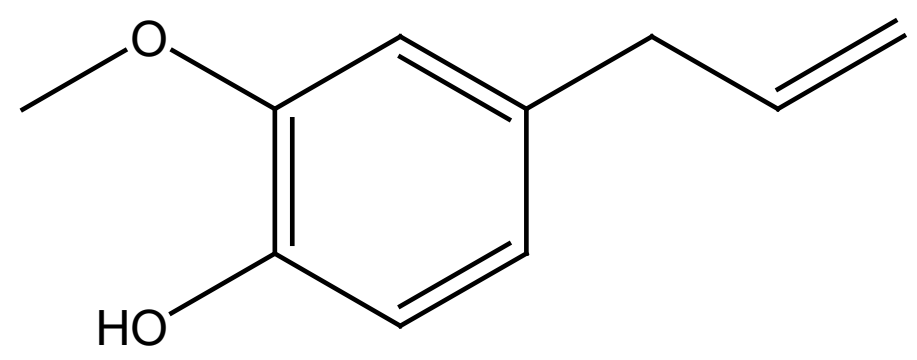

Figure 8: Eugenol - the bioactive; immunostimulatory component of Ocimum sanctum

\section{Curcuma longa (Haldi- Zingiberaceae):}

Curcuma longa L. is a perennial herb with a short stem that is widely cultivated in the tropical and subtropical countries, mainly in India and China. The powder from its Rhizome (called turmeric), in routinely use as a spice for its flavoring and digestive properties. The key biological component is curcumin (chemically, diferuloylmethane), from the class of curcuminoids, which is soluble in ethanol, acids, and chloroform, but insoluble in water (Fig. 9) [232, 233]. This is, first and foremost, a herbal medicines that was studied extensively by the modern scientific methods in the past few decades, mainly for the purposes of establishing its biological nature and pharmacological potentials. Curcumin is a yellow-orange polyphenol with diverse therapeutic applications, including anticancer, antidiabetic, antiseptic, antimicrobial, antivenom, and antiulcer activities, along with hypotensive and hypocholesterolemic effects and protection against Alzheimer's disease [234-236].

The studies of the anti-inflammatory activity of circumins have suggested that the plant can be applied in both acute as well as chronic cases of inflammation. Its potency was comparable to the condition induced by the popular anti-inflammatory drug phenylbutazone [237]. The volatile oil fractions, as well as the extracts of $C$. longa from petroleum ether, alcohol, and water, possess anti-inflammatory effects [238]. The antirheumatic activity of circumin was also established by several clinical trials in which patients treated with circumin experienced a significant recovery from symptoms. The curcumin ingredient which produces the yellow color alters the function of NF- $\mathrm{BB}$, which inhibits angiogenesis. Curcumin increases tumor cell apoptosis and decreases cell growth rate [237]. The ability to cause apoptosis in cancerous cells such as the skin, colon, duodenal, and ovary cells in an animal model was considered to be the mechanism of its anti-cancer effects $[239,240]$. It can reduce the occurrence of cancer by suppressing the effect of mutagens and carcinogens of tobacco smoke and chemicals [241]. Turmeric is been a good gastric protectant, especially against mucosal irritation, as it is a potent inhibitor of the Cox-2 pathway. Additionally, it is valuable for the treatment of Alzheimer's disease, as curcumin was found, to some extent, to reduce the accumulation of amyloid-beta oligomers.

Turmeric is a potent antioxidant as it can lower the lipid peroxidation in cells by sustaining the activities of antioxidant enzymes, such as catalases, superoxide dismutase, and glutathione peroxidase at higher levels $[242,243]$. Curcumin can induce the synthesis of heme oxygenase-1, which is another mediator of antioxidation [244]. As lipid peroxidation is a key factor in the mediation of inflammation, cardiac diseases, and cancerous conditions, the ability of curcumin to limit this peroxidation may impart its anti-inflammatory, cardiac protective, and antitumor properties. Curcumin has its regulatory role in cytokine secretion from immune cells thereby exerting both its anti-inflammatory and immunomodulatory roles (Fig. 10). In various in vitro studies, it was found to inhibit the proinflammatory-responsive Th1 cytokine, which conferred beneficial activity in the regulation of Th-1controlled immune disorders [245]. It was also reported to inhibit the production and function of inducible nitric oxide synthase from activated macrophages. Curcumin can limit the secretion of proteolytic enzymes, especially collagenase, elastase, matrix metalloproteinases, and hyaluronidase, which are released from macrophages after activation [246].

Curcumin is a good modulator of lymphocyte-mediated immune functions, especially $\mathrm{CD} 4^{(+)} \mathrm{T}$ cells, and B cells. It can regulate B cell lymphomas by arresting growth and inducing apoptosis more effectively than normal B lymphocytes [247]. Turmeric increased the mitogenic response of splenic lymphocytes [248]. Curcuma, a lipopolysaccharide from roots of turmeric is similar to bacterial lipopolysaccharide in immunostimulation [249]. Dietary curcumin found to increase IgG levels in rats 
[250]. Recently it was found that curcumin alone can protect BALB/c mice from lung inflammation caused by Klebsiella pneumonia [251]. Curcumin has been a primary ingredient in pharmaceutical products for anti-aging and ophthalmic therapies and cosmetic preparations, but the main limitation for the development of curcumin as an absolute remedy in treatment and prevention is its reduced oral bioavailability [252]. Alagawany et al. [22] noted that the supplementation of rabbit diets with turmeric significantly enhanced serum $\operatorname{IgG}$ and $\operatorname{IgM}$ values $(\mathrm{P}<0.05)$ and concluded that the treatment with herbs that contain flavonoids, such as turmeric, which have similar activity as vitamin $\mathrm{C}$ and have antioxidant properties that can improve immune functions.

Pharmacological studies based on recent advances, such as cheminformatics, have investigated various phytoconstituents in $C$. longa, such as diphenylheptanoids. These studies, which elaborately investigated the absorptive, metabolic, and toxic properties of these constituents revealed the dosedependent hepatotoxicity of diphenylheptanoids [253]. A major limitation of the therapeutic application of curcumin is its poor intestinal absorption, to the extent that some novel curcumin-based combinations (e.g., curcumin-impregnated soluble dietary fibre dispersions) have been introduced, which possess high bioavailability and maximum absorption when compared with unformulated curcumin [254, 255]. The various metabolites from curcumin include dihydrocurcumin (DHC), tetrahydrocurcumin (THC), curcumin glucuronide, curcumin sulfate, among which tetrahydrocurcumin forms one of its primary metabolites that is not found in turmeric. Also, certain other curcumin analogs, such as demethoxycurcumin and bisdemethoxycurcumin, were shown to exhibit better biological activities than that of curcumin, and can be applied as drug candidates with almost no side effects [256].<smiles>COc1cc(/C=C/C(=O)CC(=O)/C=C/c2ccc(O)c(OC)c2)ccc1O</smiles>

Figure 9: Structure of curcumin Keto-form 


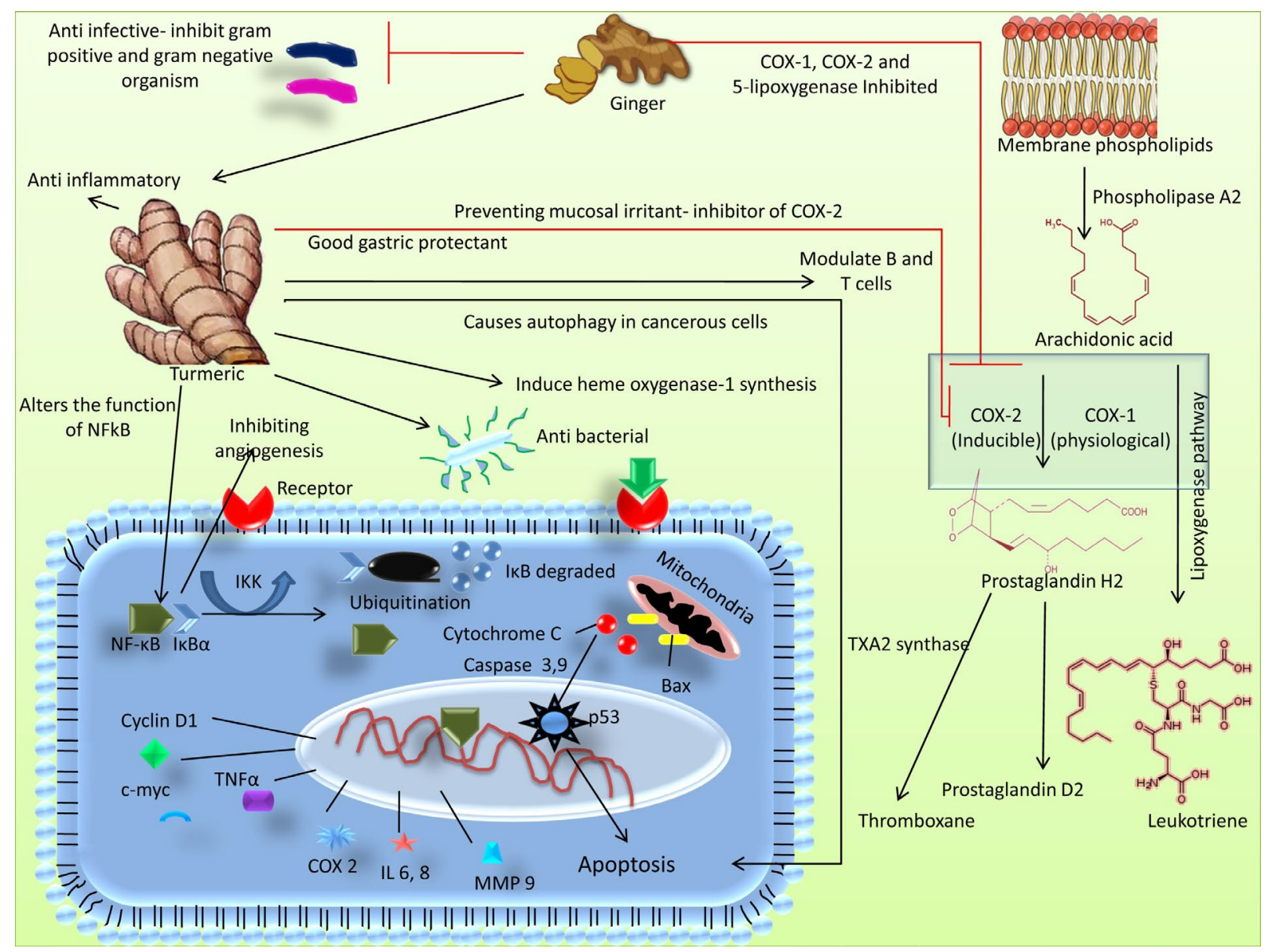

Figure 10. Immunomodulatory effects of turmeric and ginger

\section{Zingiber officinale (Ginger - Zingiberaceae)}

Ginger is the rhizomic part of Zingiber officinalis, one of the most widely used members of the Zingiberaceae family, which has a very long therapeutic history over approximately 2500 years. Ginger has been used since the advent of medicine for disparate illnesses affecting mankind throughout world: to rectify digestive perturbations such as gastric upset, nausea, and diarrhea. In addition to its culinary use in daily dishes, it has also been used in beverages and various dietary preparations in different countries, and particularly in India and China [257]. Zingiber officinale, its underground stem, has been used in Indian and Arabic traditions as a medicament [258]. It has been used in China for more than two centuries and in Mediterranean and Western countries for the treatment of headaches, cold, nausea, arthritis, rheumatism, and muscular discomfort [259-261]. Altogether approximately 400 different biologically active compounds have been characterized from the ginger rhizome, the major constituents are carbohydrates (50-70\%), lipids (3-8\%), and oleoresins (4-7\%). Oleoresins include a class of structurally related compounds called gingerols along with other oleoresin homologs, such as shogaol, zingerone, and volatile oils [262, 263]. Certain studies have been conducted on the pharmacokinetic aspect of ginger; one study indicated the half-life of gingerol, the active constituent of fresh ginger, was 7.23 min when administered intravenously in mice [264, 265].

Ginger is mentioned as 'great medicament' in Ayurveda, and this very warming herb is highly useful as a carminative, antipyretic, and for the treatment of bronchitis, diarrhea, autoimmune encephalomyelitis, and rheumatism. The protective activity of ginger against gastric ulcers was recorded [266]. The analgesic and anti-inflammatory actions of ginger extracts were also demonstrated [267], but had been acknowledged for centuries. During the last four decades, different studies have provided a validation to the already postulated thought that the anti-inflammatory properties of ginger resulted from ingredients that have anti-inflammatory action $[268,269]$. The prostaglandin biosynthesis inhibition was a novel finding of its anti-inflammatory properties in the early 1970s, which has been soundly established 
over the years. Zingiber officinale suppresses prostaglandin synthesis by inhibiting COX-1 and COX-2 enzymes. Moreover, ginger is known for its ability to subdue leukotriene biosynthesis by the downregulating 5-lipoxygenase. Although ginger has an anti-inflammatory activity similar that of nonsteroidal anti-inflammatory drugs (NSAIDs), its ability to inhibit 5-lipoxygenase distinguishes it from NSAIDs. The discovery of the ginger-induced inhibition of both COXs and 5-lipoxygenase heralded the possibility of improved pharmacological potential and reduced adverse effects than NSAIDs [257, 260]. In mice experimentally-induced with autoimmune encephalomyelitis, the administration of ginger extract modulated of IL-12 and TGF- $\beta$ expression in the serum and CNS of mice [270].

Ginger is well known for its anti-infective effects, where both gram-positive and gram-negative species growth is inhibited in the ethanolic extract. Citral, a sesquiterpene, and curcumene which are the active components in ginger oil successfully inhibited the growth of Rhizoctonia solani [271]. Standardized ginger extracts are also found to reduce bacterial load and suppress acute and chronic inflammation in Helicobacter pylori-infected Mongolian gerbils [272]. Recent studies on ginger have indicated that its antioxidant, antiplatelet, anti-inflammatory, hypotensive, and hypolipidemic potential in vitro is appreciable [273, 274]. Similarly, trials on human subjects at 5 -g doses have also verified its considerable antiplatelet activity [275]. As an immunostimulant agent, non-specific immunity was enhanced by the addition of Zingiber officinale addition [276]. A high level of ginger essential oil (200 $\mathrm{g} / \mathrm{kg}$ diet) enhanced immune functions in comparison with the low $(50 \mathrm{~g} / \mathrm{kg}$ diet $)$ and medium $(100 \mathrm{~g} / \mathrm{kg}$ diet) levels [277]. Salient immunomodulatory effects of ginger are shown in Fig. 10.

\section{Allium sativum (Garlic, Lasun - Liliaceae)}

Allium sativum (Garlic) is renowned throughout the world for its unique flavor in addition to its therapeutic properties; it has a centuries-long history for use as a therapeutic agent [9]. Garlic is a strongly aromatic bulb crop which has been in use from approximately 3000 B.C. A. sativum, often called the 'the herbal antibiotic', has been used as an anthelmintic, immune modulator, and antiseptic [278, 279]. Garlic comprises mainly sulfur compounds (approximately 33), various enzymes, amino acids, and minerals including selenium [280]. In 1920, it was found that the antibiotic constituents in garlic called "alliin" (Fig. 11), which get transformed into allicin by the enzyme allinase, in turn acts as a potent antibiotic that can limit even Mycobacterium spp., Cryptococcal meningitis, Trichophyton mentagrophytes, Candida albicans, and the influenza virus [281, 282]. Allicin (diallyl-thiosulfinate), along with other sulfur conjugated compounds are considered to be the major components responsible for the antimicrobial property of by garlic [283]. An in vitro assessment of the virucidal activity of the various phytoconstituents in A. sativum showed the effectiveness of the anti-viral property was a maximum in ajoene, followed by allicin, then allyl methyl, thiosulfinate, and least effective in methyl allyl thiosulfinate [284]. The aqueous, ethanolic, and chloroformic extracts were proven to be effective against a wide range of pathogens, including gram-negative, gram-positive, and Mycobacterium sp. [285]. Garlic is also known for its antifungal action which is attributed to the presence of another phytoconstituent Ajoene [286].

Garlic has been proven to be a wonderful medicinal plant with its role as an effective therapeutic agent in cardiovascular diseases, regulation of cholesterol, blood pressure, and blood sugar levels, in addition to having effective antibacterial, antiviral, antifungal, and antiparasitic actions [287, 288]. Garlic also exerts immunostimulatory properties, antioxidant features, and antitumor actions owing to its unique chemical constituents, of which there are approximately 200. Compounds such as the flavonoids, cyanidin and quercetin, and vitamins $\mathrm{A}, \mathrm{C}$, and $\mathrm{E}$, are the constituents of garlic that provide protective action from oxidants and free radicals [279]. The aqueous extract of garlic displayed a dose-dependent increase of oxidative activity and augmented T-lymphocyte blastogenesis [289]. Aged garlic extract has similarly boosted the peripheral blood lymphocytes' cytotoxic activity, which indicated the immunostimulant function [290]. Eikai et al. [291] studied multiple functions of aged garlic extracts, which demonstrated a reduction in allergic Ig E production, enhanced NK cell activity against tumors, and support in in overcoming stress-induced immunosuppression. Recently, immunomodulatory components from raw garlic called 'garlic agglutinin' and 'lectins' were identified and their effects on immune cells were studied [292]. In a study on growing rabbits, Alagawany et al. [22] postulated that dietary garlic supplement could improve the immune system owing to the elevation in the immunoglobulin concentrations (IgG and $\operatorname{IgM}$ ) of rabbits versus the control diet. A study in humans, of 
the supplementation of aged garlic extracts $(2.56 \mathrm{~g} / \mathrm{d}$ for $90 \mathrm{~d}$ during the season of cold and flu), improved the function of the immune system and reduced the severity of colds and flu [293]. Moreover, the addition of garlic $(250 \mathrm{mg} / \mathrm{kg})$ enhanced the immune responses of both adult and weaning rats [294]. Nutrigenomic studies performed over 280 days old Ven Cobb broilers suggested that a diet supplemented with garlic powder and Ocimum sanctum leaf powder improved the T-cell mediated immunity and realtime PCR confirmed the increased expression of TLR 2, TLR 4, and TLR 7 mRNA levels in the broiler blood and supported the growth parameters and immunity [295]. Garlic possess various potent biologically active constituents and therefore, a vital role in nutraceutical applications is warranted, for which extensive and focused further research is required. An overview on the immunomodulatory actions of garlic is depicted in Fig. 12.

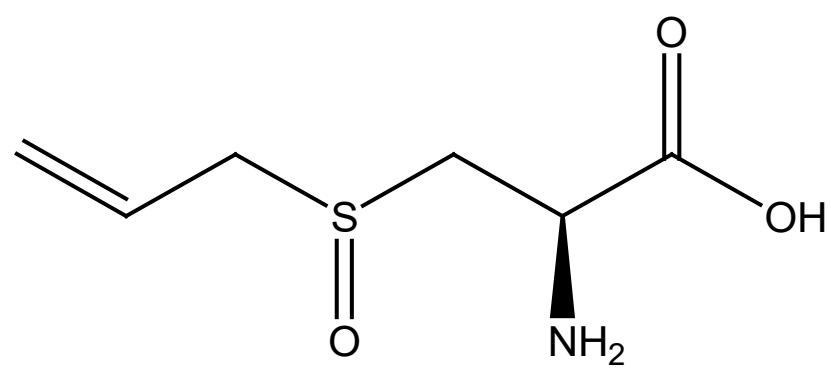

Figure 11: Chemical structure of Alliin; a sulfoxide natural constituent of garlic.

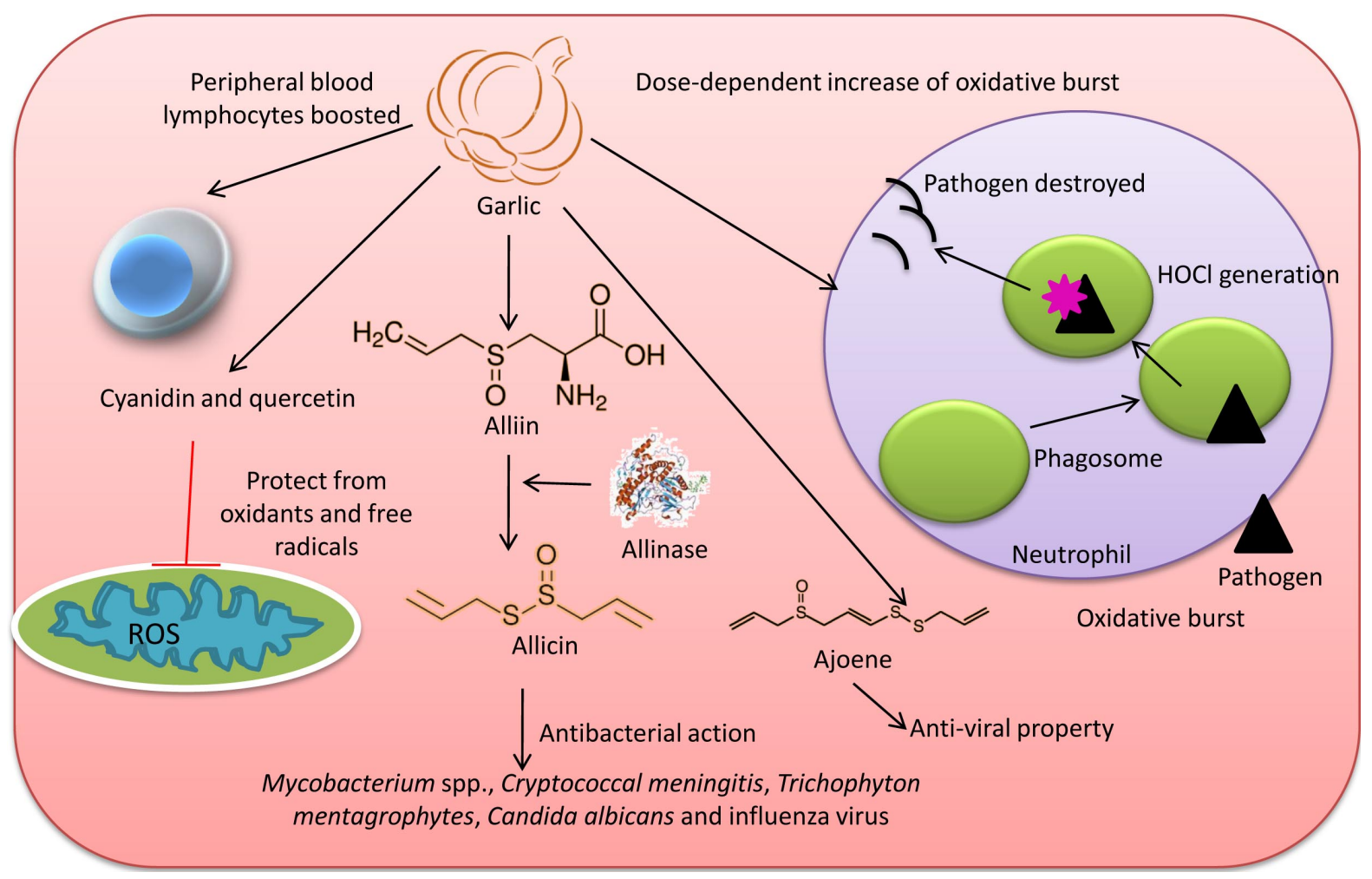

Figure 12. Immunomodulatory actions of Garlic

\section{Black cumin (Nigella Sativa - Ranunculacease)}

The seed of Nigella sativa is a small dicotyledon with many common names and belongs to the botanical family of Ranunculacease [296]. The black seed has been used as a natural medicine for several diseases for over 2000 years. $N$. sativa seed has several chemical constituents: mucilage, thymoquinone, crude 
fiber, reducing sugars, resins, alkaloids, flavonoids, organic acids, sterols, tannins, saponins, and proteins. It is acknowledged that the biological action of $N$. sativa seeds arises from its high content of unsaturated fatty acids, especially linoleic acid (55.6\%), oleic acid (23.4\%), and palmitic acid (12.5\%) [297, 298]. Several studies confirmed black cumin as an alternative source of antibiotics and vaccines in the enhancement of poultry immunity and declining mortality owing to to immunomodulatory and therapeutic activities [299-303]. Thymoquinone present in the herb as reported to exert inhibitory action over LPS-induced fibroblast proliferation and $\mathrm{H}_{2} \mathrm{O}_{2}$-mediated generation of reactive oxygen species [304].

Many studies reported that $N$. sativa inhibited many inflammatory mediators, including prostaglandins and leukotrienes, amended splenocyte proliferation, Th1/Th2 cytokine profile, macrophage function. and NK anti-tumor activity [20, 305-308]. Akhtar et al. [309] indicated that mortality was reduced from $16.67-4.17 \%$ by the supplementation of $1.5 \%$ black cumin to layered diets. Mansour et al. [310] notice that viability rate of broilers fed a diet containing 1\% powdered Nigella sativa seeds were maximized by $50 \%$ compared to the control group. According to Al Jabre et al. [311], the volatile oil from $N$. sativa has 67 constituents with have antibacterial activity against species such $E$. coli and Staphylococcus. The active ingredients of $N$. sativa exert antioxidant, antiproliferative, antibacterial, and anti-inflammatory effects, which induce positive impacts on the immunity and organs involved [312-314]. Toghyani et al. [315] showed that the antibody titer against Newcastle Disease (ND) and Infectious Bursal Disease (IBD) significantly rose as a result of the substituting grounded $N$. sativa seed for bacitracin methylene disalicylate in diets of the broilers. The progress in the titer of IBD and ND resulted from black seed oil constituents such as thymol, nigellicine, and thymoquinone [52, 316, 317]. Toghyani et al. [315] also observed an increase in the weight of lymphoid organs when broilers were fed diet that contained $0.2 \%$ and $0.4 \%$ black seeds. In contrast, the addition of $N$. sativa seeds had no significant influence against ND and influenza virus at 18 and 28 days on antibody titers, heterophil to lymphocyte ration, and albumin to globulin ratio.

Many studies were conducted using experimental animals to study the anticancerous effects of $N$. sativa. The anticancerous properties have been attributed to the bioactive components in black cumin. ElKadi and Kandil [317] observed an enhanced activity of Natural Killer (NK) cells, of 200-300\%, in patients with advanced cancer after receipt of a multi-modality immunotherapy along with $N$. sativa seeds. Salomi et al. [318] claimed that black seed extract inhibited skin carcinogenesis in mice induced by dimethyl $[\alpha]$ anthracene/cotton oil, decreased the number of papillomas per mouse, and delayed the onset of papilloma formation. Moreover, Iddamaldeniya et al. [319] reported that black cumin inhibited the development of two leukemic cell lines and five solid tumor cell lines. Musa et al. [320] demonstrated that ethanol extracts derived from black seed could inhibit ehrilich ascites tumor development through minimization of the cell count as well as inhibition of tumor development. Various extracts of black seed have shown differential toxicity on cell lines, for instance, the majority of the essential oils produced cytotoxic effects in the P815 cell lines compared with the ethanolic and butanol acetate extracts [321], whereas the ethyl acetate extracts showed increased cytotoxicity versus the BSR line of cells. Furthermore, Mbarek et al. [306] found that the utilization the black seed essential oil onto the tumor site enhanced the livability of the mouse and prevented liver metastasis incidence. Through the p53dependent pathway, thymoquinone could cause the death and destruction of cancerous cells by apoptotic mechanisms in the case of human colorectal cancer [322, 323]. Investigations revealed that the aqueous suspension of $N$. sativa involving volatile oil and fixed oil exerted antiulcer potential in curing gastric ulcers in Wistar albino rats [324, 325]. In a trial conducted in male albino rats, the outcome suggested the beneficial protective role of black seeds oil and thymoquinone versus cyclophosphamide-induced toxicity and recommended the clinical use of $N$ sativa as a supportive anticancer remedy to minimize the side impacts of long-term chemotherapy [326]. Black cumin seed plays a major role as anticancer agent combined with high level of thymoquinone oil, in addition to the inhibition of the NF- $\mathrm{KB}$ signaling pathway [327].

\section{Cinamomum spp. (Cinnamon)}

Cinnamon, a tropical evergreen plant, has been used by diverse cultures around the globe for several centuries as a common spice. It is obtained from the genus Cinnamomum and falls into two main varieties; Cinnamomum zeylanicum and C. cassia. Traditionally cinnamon has been regarded as a remedy 
for general ailments related to respiratory, digestive, and gynecological systems. In vitro and in vivo studies conducted in various parts of the world have reported advantageous therapeutic potentials of cinnamon, especially C. zeylanicum, [328-330]. Even though the tree exhibits curative or culinary properties in nearly all of its parts, the extracted volatile oils from different parts of the tree showed considerable variation in composition, thus indicating a variation in pharmacological activity as well [331-335].

The pharmacokinetics-based absorptive in vivo study to measure procyanidin B-2 and procyanidin B-3, isolated from Cinnamomum bark, after oral administration was conducted in rats [336]. Cinnamaldehyde, another constituent, was detected in plasma very early and retained for an extended time after intradermal administration in dogs. The pharmacokinetics of cinnamic acid were evaluated in rats as a comparison among a decoction of ramulus Cinnamomi and pure cinnamic acid; the results showed that the plasma concentration, as well as bioavailability of cinnamic acid, were higher in the decoction group than those in the pure group [337].The major metabolic pathway adopted by Cinnamomum constituents was shown to be by the oxidation to the corresponding cinnamic and phenyl propionic acids, which were predominantly excreted as glycine conjugates. Based on the in vitro evaluation, Cinnamomum constituents were also proven to inhibit hepatic microsomal cytochrome P450 [338].

In addition to the enormous use of the bark for its taste as a flavor in tea, it has also been indicated as antimicrobial and anti-inflammatory agent, especially $C$. zeylanicum, which has shown potential anti-microbial action against with potent bacteria such as Acinetobacter, Bacillus sp., Brucella melitensis, Clostridium difficile, C. perfringens, Enterobacter sp., E. coli, Helicobacter pylori, Klebsiella pneumonia, Listeria, Mycobacterium sp., Salmonella sp., Staphylococcus, Streptococcus, and Yersinia enterocolitica along with antifungal action towards Aspergillus fiavus and A. fumigates [329, 333]. Cinnamon possesses antiparasitic effects, which have also been established by various studies, especially with regard to those mosquito species, such as Aedes and Culex [339, 340]. Chao et al. [341] identified different bioactive components in the leaf extracts of $C$. osmophloeum and demonstrated the antiinflammatory effects, which indicated a potential therapeutic action for chronic inflammation. The polyphenol extracts are found to overcome inflammation by modulating pro- and anti-inflammatory cytokine gene expression in mouse macrophages [342]. Cinnamon extracts also modulate Cytotoxic-T cells and helped in the suppression of tumor progression [343].

Studies conducted by Bandara et al. [344] and Ranasinghe et al. [345] have determined that cinnamon has a therapeutic potential for diabetes. Its actions include the regulation of weight loss related to diabetes, limiting of the fasting blood sugar level, a reduction in LDL and promotion of HDL cholesterol, augmentation of the insulin levels, along with considerable protective effects towards associated diabetic neuropathy and nephropathy [346]. Antioxidant effects have been proven with the essential oils extracted from the bark of $C$. zeylanicum, which decreased the formation of 3-nitrotyrosine and inhibited lipid peroxidation induced by peroxynitrite [347]. This was also potent in scavenging the free radicals (especially in antioxidant testing using DPPH and ABTS radicals), including ROS, RNS, RSS, and cations [335, 348].

\section{Aloe vera (Ghrita kumari - Liliaceae):}

Aloe vera is popular in Indian Ayurveda, as well as several other cultures, and has been used for millennia, including traditional Chinese, Greece, Egyptian, Mexican, and Japanese medicines as the plant is renowned for its use as a medicinal or cosmetic product. The name is originally derived from the Arabic language "Alloeh" which means shining bitter substance and "vera" that means true in Latin. Centuries back the Greek scientists considered the Aloe plant as the universal panacea while the Egyptians regarded it as the plant of immortality [349, 350]. Its gel is used as a laxative, antihelminthic, and hemorrhoid remedy. Along with licorice, it is considered as a great cure for skin infections, such as psoriasis. In modern days, A. vera is a major ingredient in many lotions and cosmetics [351-353]. Mucopolysaccharides present in the aloe keeps the skin moisturized, along with its skin softening property owing to the amino acids, thereby facilitating anti-aging effect. The presence of biogenic material keeps $A$. vera as a medicine related to gerontology and rejuvenation of skin so that it has been extensively used as a skin tonic in cosmetic industry [354, 355]. 
As anthracene hydroxyl derivatives are the major chemical constituent of this plant, the effectiveness of Aloe in wound healing occurs through the presence of a polysaccharide called Glucomannan and a growth hormone (gibberellin), which interacts with receptors for growth factor on the fibroblast, for its proliferation, and significantly aids in collagen synthesis [356]. $\beta$-Sitosterol was also identified as one of the constituents of aloe vera mucilage that can facilitate angiogenesis and further healing of traumatic conditions along with the genetic expression of VEGF in the affected tissues [353, 357]. Aloe latex holds different anthraquinone glycosides such as aloin, aloe-emodin, and barbaloin, which are potent laxatives also (Fig. 13). Pharmacodynamic studies show that these glycosides possessed a biological half-life of $48-50 \mathrm{~h}$ and digestion by intestinal microflora was required before being absorbed into systemic circulation, followed by their elimination through orogastric routes as well as breast milk [358, 359].

Various studies have clearly demonstrated its anti-inflammatory effects both in vitro and in vivo. The aloe plant possesses the peptidase bradykinase, which can breakdown bradykinin and reduced pain. Another anti-inflammatory compound present, is C-glucosyl chromone, which is an inhibitor of the cyclo-oxygenase pathway and thereby blocking the prostaglandin E2 production from arachidonic acid $[355,360,361]$. The polysaccharide component of the gel showed adjuvant activity for antibody production and delayed type hypersensitivity in mice. In a study by Stuart et al. [362], acemannan, the active carbohydrate from $A$. vera gel increased the respiratory burst of macrophages and phagocytic activity. Aloe ingredients, aloin, and aloe-emodin are found to have an anti-inflammatory effect by reducing prostaglandin $\mathrm{E}$ and nitric oxide synthesis in macrophages [363]. Recently, Im et al. [364] confirmed immunomodulatory functions of processed $A$. vera in gel orally administered in mice. The results of the studies on high-fat diet-induced diabetic mice showed a reduction in growth of Candida albicans and an increased proliferation of cytotoxic $\mathrm{T}$ cells. The reduction in pain and growth prevention of the different bacteria and fungus on the skin occurred owing to the salicylic acid present in the $A$. vera plant $[358,365]$.<smiles>O=C1c2c(O)cccc2C(C2OC(CO)C(O)C(O)C2O)c2cc(CO)cc(O)c21</smiles><smiles>O=C1c2ccccc2C(=O)c2cc(CO)ccc21</smiles>

Figure 13: Alove vera Aloe ingredients, A. aloin and B. aloe-emodin

\section{Phyllanthus emblica (Emblica officinalis, Amla - Euphorbiaceae):}

P. emblica commonly called as amla is believed as the first tree to be created according to the ancient Indian mythology and serves as vitamin $\mathrm{C}$ source. The active ingredient in Amla named Phyllemblin has substantial pharmacological actions along with other bioactive compounds like phyllaemblic compounds, quercetin, gallic acid, flavonoids, tannins, pectin and polyphenolic molecules [366-368]. Owing to the presence of bioactive compounds including Emblicanin-A, Emblicanin-B, Pedunculagin, and Punigluconin, the amla extract is the main constituent of many ayurveda tonics recommend for rejuvenation, and recuperation, etc. [369]. The useful applications of P. emblica have been identified for conditions such as cancer, diabetes, liver and heart disorders and anemia, among others. Amla is reported to have numerous bio-related activities such as antioxidant, anti-inflammatory, antipyretic immunomodulatory, growth promoter, gastroprotectant, cytoprotectant, hypoglycemic, hypolipidemic, memory enhancer and protective against arsenic-induced liver toxicity [40, 368, 370- 
373].The above mentioned biological activities at large and antioxidant, in particular, has been utilized in cases of oxidative stress since it can preserve the bioavailability of nitric oxide [374]. It also exerts an anti-platelet effect through the endothelial cells, and platelet-derived nitric oxide [375]. The interactive pharmacodynamics evaluation between $P$. emblica and other anti-platelet drugs e.g. clopidogrel and ecosprin in type II diabetes mellitus patients indicated that the enhanced antiplatelet activity in mediating oxidative stress compared with the application of these drugs individually [376].

The anti-inflammatory and analgesic properties of amla are similar to the non-steroidal antiinflammatory drugs (NSAIDS), i.e. through the inhibition of inflammatory mediators [377]. Amla has an effective antibacterial activity against various bacterial strains including E. coli, Klebsiella, Proteus, Pseudomonas aeruginosa and Salmonella sp. [378, 379]. A long-term supplementation of dietary Amla has been found effective against to overcome infection of Klebsiealla pneumonia infection in mice along with an elevated phagocytic activity [380]. Its immunomodulatory functions are well observed via NK cell activity and ADCC mechanism to control and regress tumor growth in mice [381]. Amla use has significantly reduced the inflammation and edema in rats induced with arthritis, indicating that it can be adopted for arthritic therapy [371, 382]. Amla was reported to have its protective impact on hypercholesterolemia induced cardiac diseases through hypolipidemic effects in experimentations with hypercholesteremic rats [383]. Paul and Khanna [384] pointed out that Phyllanthus emblica Linn. played an important role as a natural Immunostimulant, stimulating nonspecific immune mechanisms. Emblica officinalis contains many vitamins like vitamin $\mathrm{C}$ and $\mathrm{A}$, etc. and regular use of this plant enhances the immunity and fights cancers in the body [223].

\section{Moringa (Moringa oleifera)}

Moringa is a traditional herb and one of the essential members to be mentioned with priority in medicinal plants. With multiple medicinal properties, it is a folk remedy for various health-related conditions including cancer, catarrh, skin disease, gastric ulcer, nervous condition, lower blood sugar, and diabetes. It is also used to strengthen eye, brain, liver, gall and immune system. Moringa is also used to expel the intestinal worm. Moringa is also considered a significant source of various bioactive compounds including riboflavin, vitamins $\mathrm{A}$, folic acid, nicotinic acid, ascorbic acid, pyridoxine, iron, beta carotene, calcium and $\alpha$-tocopherol [385]. Akhouri [386-388] theorized that Moringa plant is working through the immune system. Aqueous extract of $M$. oleifera leaves lowered the expression of NF- $\mathrm{kB}$ and enhanced the cytotoxic effect against the pancreatic cancer cells [389]. Three kinds of extracts viz., alcoholic, hot and cold extracts of $M$. oleifera can be used for therapeutic applications. As compared to the standard Diclofenac Sodium, an alcoholic extract of M. oleifera has exerted an anti-inflammatory effect. However, the Minimum Inhibitory Concentration (MIC) of the alcoholic extract was evaluated as low on coldwater as well as hot water extracts of $M$. oleifera. The coldwater extract was effective in counteracting Gram-positive bacteria like $P$. aeruginosa while hot water extract and alcoholic extracts were found to be more active against Gram-negative bacteria. Gupta et al. [390] worked on the immunomodulatory impact of ethanolic extract of $M$. oleifera leaves on normal and immunosuppressed mice model. Gupta and coworkers noticed that the $M$. oleifera treated group revealed a significant increase $(\mathrm{P}<0.05)$ in hematological parameter, phagocytic index and serum enzyme level. In another study, Bukar et al. [391] investigated the antimicrobial profile of $M$. oleifera extract and reported that the chloroform-ethanol extracts showed a sanitizer property because it acts as an antimicrobial agent.

\section{Morinda citrifolia (Noni- Rubiaceae)}

Morinda citrifolia is widely known as "Noni". Other names include Indian mulberry, Nonu/Nono, Chinese fruit or Nhau in various parts of the world. It is available in ready to use form such as capsules, juice, and tea [392]. It belongs to the family Rubiaceae and grows in the coastal areas of many countries. In India, it is found in Andaman and Nicobar group of Islands. This plant has been used by Polynesians for around 2000 years for its various medicinal properties [393]. It has tremendous immunomodulatory actions on humans, animals and poultry species. Noni has been used for the prevention and treatment of many diseases such as diabetes, hypertension, cancer, etc. Noni fruit extract has potential anti-oxidant and free radical scavenging property due to the presence of functional components which are to be still identified [394]. A similar effect has been noticed in smokers where the use of noni fruit juice was able to mitigate oxidative damage to their DNA hence thereby could protect 
occurrence of cancer [395]. Also, during an experiment with mice peritoneal macrophages its fruit juice treatment in vitro was found to increase nitric oxide (NO) production thereby increasing their killing ability on Leishmania amazonensis amastigotes [396]. Noni fruit also has an anti-inflammatory action due to the inhibition of myeloperoxidase, TNF- $\alpha$, IFN- $\gamma$ activity in intestines hence is useful in the treatment of many inflammatory disorders such as inflammatory bowel disease where it maintains the integrity of intestine [397]. Even its leaf possesses anti-inflammatory activity and has been used to treat pain and inflammation in folk medicine [398]. M. citrifolia fruit also affects adaptive immune B and T cells thereby potentiates both humoral and cellular immune system [399]. It has shown anti-bacterial actions against various bacteria viz. Enterococcus faecalis, Bacillus subtilis, Escherichia coli, mycoplasmas, etc. [400, 401]. The immunostimulatory property is due to the polysaccharide fraction from the fruit of this plant [402]. Fruit extract of Noni has also been demonstrated to inhibit the growth of Candida albicans under in-vitro condition [403]. Leaf extract and anthraquinones in the bark of noni possess anti-viral activity against certain viruses such as hepatitis $C$ virus, influenza viruses, etc. [404, 405]. Thus, the immunomodulatory capacity of this multi-purpose herbal plant leads to anti-cancer, antiinflammatory, anti-helmintic, antibacterial, antiviral, antifungal, and analgesic action.

\section{Picrorhiza kurroa (Kutaki - Scrophulariaceae):}

Picrorhiza kurroa is a renowned herb belonging to the Scrophulariaceae family with a remarkable reputation among the traditional medical practitioners. The plant is accepted as a hepatoprotective agent with its bioactive components like picrosides, kutkoside and catapol (Fig. 14) [406, 407]. Its antiinflammatory, antioxidant, immunomodulatory and antineoplastic actions have been proven [408, 409]. The anti-cancer property may be attributed to those metabolites of $P$. kurroa that render antioxidant and cytotoxic activities. An iridoid glycoside named Picroliv obtained from the root and rhizome of $P$. kurroa has anti-allergic along with immune boosting potentialities [410]. The methanolic, and aqueous extracts have been recognized with antioxidant, and anti-neoplastic potential, thus paving the way for the future clinical application [409, 411, 412]. In Indian traditional medicine, $P$. kurroa is a constant candidate for hepatic diseases and has been proven to ameliorate the hepatic toxicities induced by chemicals such as carbon tetrachloride, d-galactosamine, paracetamol, and thioacetamide in various in-vivo studies. These scientific bases led to the genesis of an Ayurvedic formulation named 'Arogyavardhini' with $50 \% P$. kurroa as a remedy for viral hepatitis [413].

Various immunomodulatory functions of the plant show that its extracts enhance delayed type hypersensitivity (DTH), antibody production and phagocytosis [409, 414-416]. Picroliv also protected hamsters from challenge with Leishmania donovani promastigotes [417] and found to enhance antibody titer, plaque forming cells and DTH response to sheep RBC when orally administered in mice [417]. Clear evidence of immune stimulation obtained from a bi-polymeric extract of $P$. kurroa, which enhanced HA titer, DTH response, phagocytic index and CD4/CD8 response when used with sheep RBC in a dose-dependent manner [415]. Humoral antibody response was improved with oral administration of an ethanolic and aqueous extract of the rhizomes of Picrorrhiza kurroa [418].

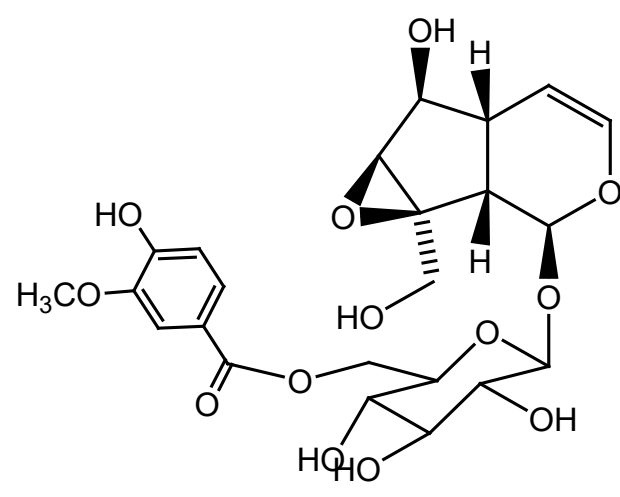

A

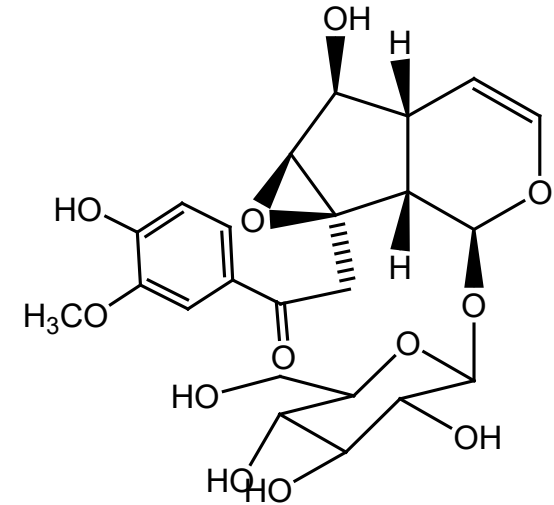

B

Figure 14: A. Picroside I and B. Kutkoside: Picrovile which is antiallergic and the immune booster is a mixture of the two in $1: 1.15$ ratio. 
Andrographis paniculata (Kirayat - Acanthaceae):

Andrographis paniculata is one among the extensively used traditional plants, worldwide. Being a native of peninsular India and Srilanka, commonly known as King of Bitters or kalmeg, an annual herb. It is also distributed in Southeast Asia and West Indian Islands [419, 420]. Several active compounds such as labdane diterpenoid lactones and flavonoids have been extracted from $A$. paniculata through phytochemical studies, revealing its pharmacological properties [421, 422]. This has been traditionally used as a remedy for various infectious diseases and gastric problems like colic pain, loss of appetite, flatulence, diarrhea, pruritis, skin diseases and wounds [422, 423].

Bioactive compounds like andrographolides and arabinogalactan proteins from dried $A$. paniculata were proven to exhibit antibacterial and antifungal actions [424]. Andrographolides are mainly concentrated in leaves and were found protective against cellular damages due to hypoxia/reoxygenation in a time-dependent manner [425]. This mode of cell protection is effected through the upregulation of reduced glutathione (GSH) level and antioxidant enzyme activities [426]. A significant stimulation of antibody and DTH response to sheep RBCs in mice has been observed in $A$. paniculatas ethanolic extract and purified diterpene andrographolides [81]. These preparations also stimulate macrophage migration, phagocytosis of ${ }^{\mathrm{u}} \mathrm{C}$-leucine labeled E. coli, and in-vitro proliferation of splenic lymphocytes [81]. The stimulation was found to be both antigens, specific and nonspecific. It was lower with purified andrographolides than with the ethanolic extract indicating the presence of a substance(s) other than andrographolides that may be contributing towards immunostimulation [427]. Andrographolide has been reported with potent inhibitory effect on the NF- $\mathrm{BB}$ and STAT3 signaling pathways so that it could downregulate the inflammatory responses mediated by the expression of COX2 and iNOS genes [428]. The inhibitory action of Andrographolide over the TLR4 stimulated downstream signaling through $\mathrm{NFkB}$ was targeted against the insulinoma tumor and found to block the tumor progression $[429,430]$. Clinical investigations are being undertaken by various researchers and have come up with promising results, suggesting the therapeutic value of $A$. paniculata against chronic inflammatory and immune-mediated ailments e.g. Crohn's disease, rheumatoid arthritis and ulcerative colitis [431-433]. The combination of Andrographis paniculata and Tinospora cordifolia was found to increase macrophage count, neutrophil adhesion and cellular responses thus showing the immunostimulatory property of these drugs [434].

\section{Asparagus racemosus (Satawar - Asparagaceae)}

Asparagus racemosus (Shatavari) is an undershrub and a vital component of almost all traditional Ayurvedic preparations. The unique medicinal potential of Asparagus is attributed due to the presence of steroidal saponins (or Shatavarins) as well as sapogenins present in the root along with other compounds like quercetin, hyperoxide and rutin that are distributed in various parts of the plant $[435,436]$. Being used in Ayurveda as a Rasayana (that impart general well-being of the individual), this plant has its action as a nervine tonic, galactagogue, carminative, stomachic, aphrodisiac, diuretic as well as an antiseptic [437]. Its root extract is a generic antidiarrhoeal drug in traditional medicine, and that can be attributed to the inhibition of the biosynthetic pathway of prostaglandin E, thereby limiting the gastrointestinal motility and secretion. Shatavari is a protectant from gastric ulcer as it can complex with mucus at the base of ulcer thereby preventing the corrosive effects of pepsin and gastric acids. Asparagus has its action on female genital tract and hormone synthesis as evidenced by in-vivo studies. A glycoside named Shatavarin I from the root of $A$. racemosus has been found to be involved in the competitive blockage of uterine contraction induced by oxytocin in different laboratory animals [438-440].

The methanolic extract of Asparagus can limit hippocampal and neuronal damage induced by kainic acid in mice with a reduction in membranal lipid peroxidation [441]. This recovery could be due to the increase in glutathione peroxidase concentration in neuronal tissues along with elevated glutathione level that acted as the nucleophilic scavenger of harmful chemicals. The crude and purified fractions of Asparagus significantly inhibited the lipid peroxidation and regulated protein oxidation as evidenced by in vitro membrane studies by Kamat et al. [442]. The reduction in lipid peroxidation imparts a cardioprotective role since it decreases the blood lipid profiles which was already proven in an in-vivo study using hypercholesteremic rats. The antihepatotoxic perspective of $A$. racemosus is documented which describes that the alcoholic extract can reduce the elevation in liver enzymes like alanine 
transaminase, aspartate transaminase and alkaline phosphatase in certain toxic conditions like carbonate tetrachloride poisoning [443]. Extract of $A$. racemosus has been tested for anticancer activity and only showed a delay in tumor development instead of a complete prevention against the development of solid tumors [444].

A. recemosus is being recognized as a potent immunostimulant particularly with the extract from its root [445]. Marked leukocytosis along with enhanced phagocytic activity has been reported by Muruganadan et al. [443] from the decoction of its powdered root. Its alcoholic extract can improve both humoral and cell-mediated immunity that reveals it as a potent herbal adjuvant [446]. The protective effect of $A$. racemosus against myelosuppression in mice induced by cyclophosphamide as a single dose has been reported by Thatte and Dahanukar [447]. Cyclophosphamide was administered at $200 \mathrm{mg} / \mathrm{kg}$ (single dose) subcutaneously to one group of mice, while the second group with 3 doses of the drug at 30 $\mathrm{mg} / \mathrm{kg}$ intraperitoneally. Both these groups received $A$. racemosus orally for 15 days before cyclophosphamide therapy. A. racemosus by itself produced leukocytosis with neutrophilia. When compared to untreated (control) groups, A. racemosus prevented the treatment group from leucopenia produced by cyclophosphamide to varying degrees. These authors have concluded that the plant is a potent immunostimulant, with effects comparable to lithium and glucan. It may be a good candidate for evaluation in patients receiving cytotoxic drugs [448].

A. racemosus is suggested to be a potent immunoadjuvant based on the in-vivo immunization study with diphtheria, tetanus, pertussis vaccines [446, 449]. Oral supplementation of its aqueous root extract though using different doses along with vaccine antigen has increased the humoral immunity [450]. It has been reported to be devoid of antibacterial action, and in this regard, the protection accessible by $A$. racemosus in septic conditions through the regulation of macrophages indicates its inherent immunomodulatory property.

\section{Panax ginseng and P. pseudoginseng (Ginseng- Araliaceae)}

$P$. ginseng is a herbaceous plant native to Korean region of Asia which is widely used as a general health tonic. Some steroidal saponins and glycosides from this plant have been shown to provide adaptogenic property [451]. The crude extract and saponins isolated Indian Origin P. pseudoginseng also possess similar activity [452]. Saponins from Indian pseudoginseng possesses effective prophylactic and immunostimulating functions by influencing macrophage migration, antibody plaque-forming cells and haemagglutinating antibody titer against sheep red blood cells [453]. Also, P. ginseng enhances the longevity and mental ability especially in young ages [454]. This is a general herb for disorders related to cardiac conditions like hypertension, blood in the urine or stool and other hemorrhagic diseases. Moreover, this is used in dyspepsia, asthma, and headache and as an excellent aphrodisiac. It is proven for treating liver damage by harmful drugs, alcohol, and narcotics in animals and also improved liver function with alcohol-induced cirrhosis $[455,456]$.

$P$. ginseng has been shown to mitigate cancerous condition through various mechanisms like antioxidant, anti-inflammatory, and apoptosis of the related genes. Fractions of ginseng extract have been reported to possess anti-tumor activity $[457,458]$ and stimulatory activity on reticuloendothelial system [459], T- cell proliferation by Con A in-vitro [460], phagocytosis [461, 462], chemotaxis [462], augmentation of NK cell activity [463-465], enhancement of antibody forming plaques and haemagglutinating antibody titers against SRBC [466, 467], production of IL-1 [465], IL-2 [463, 465, 468], TNF- $\alpha$ [453], GM-CSF [465], increase in population of CD3, CD4, CD8 cells [460, 462, 466] and immunosuppression in virus-infected mice [469]. It appears to be an extremely promising agent- to be evaluated for immunostimulatory activity in humans. The treatment by an extract of red ginseng in mice infected with respiratory syncytial virus resulted in reduced viral load in the lung, increased production of antiviral IFN- $\gamma$, improving CD11 c + dendritic cells and CD8 + T cells [470]. Quan et al. [471] reported the immune modulating potential of Ginseng towards influenza virus. Owing to its Th2 stimulation it can be a promising herbal adjuvant towards those antigens requiring an IL4 response for their clearance [472]. Supplementation of red ginseng improved the activation $T$ and NK cells of the immune system as well as increased the survival rate [473].

The bioactive components in $P$. ginseng are constituted by the complex mixture of triterpene saponins, namely ginsenosides, which belong to the triterpenoid dammarane derivatives. The presence of ginsenosides can be regarded as the major factor for the therapeutic activity of $P$. ginseng which can 
influence the vital hypothalamus-pituitary-adrenal axis as well as immune system. Pharmacodynamic evaluations after the oral supplementation of standardized ginsenoside extract have shown that it is the hydrolyzed products of protopanaxatriol ginsenosides could be detected in the systemic circulation [474, 475]. Owing to the anticarcinogenic property of ginsenoside, ginseng has been successfully employed in adjuvant breast cancer therapy and proven to augment the physical and psychomotor performances along with the improvement of general health [476, 477]. Ginseng is the fourth topmost selling herbal medicine, globally. The majority of the ginseng products are formulated from the standardized ginsenosides; more focus needs to be directed for the maximum therapeutic elucidation of these compounds [478, 479]. Important immunomodulatory activities of Panax ginseng are shown in Fig. 15.

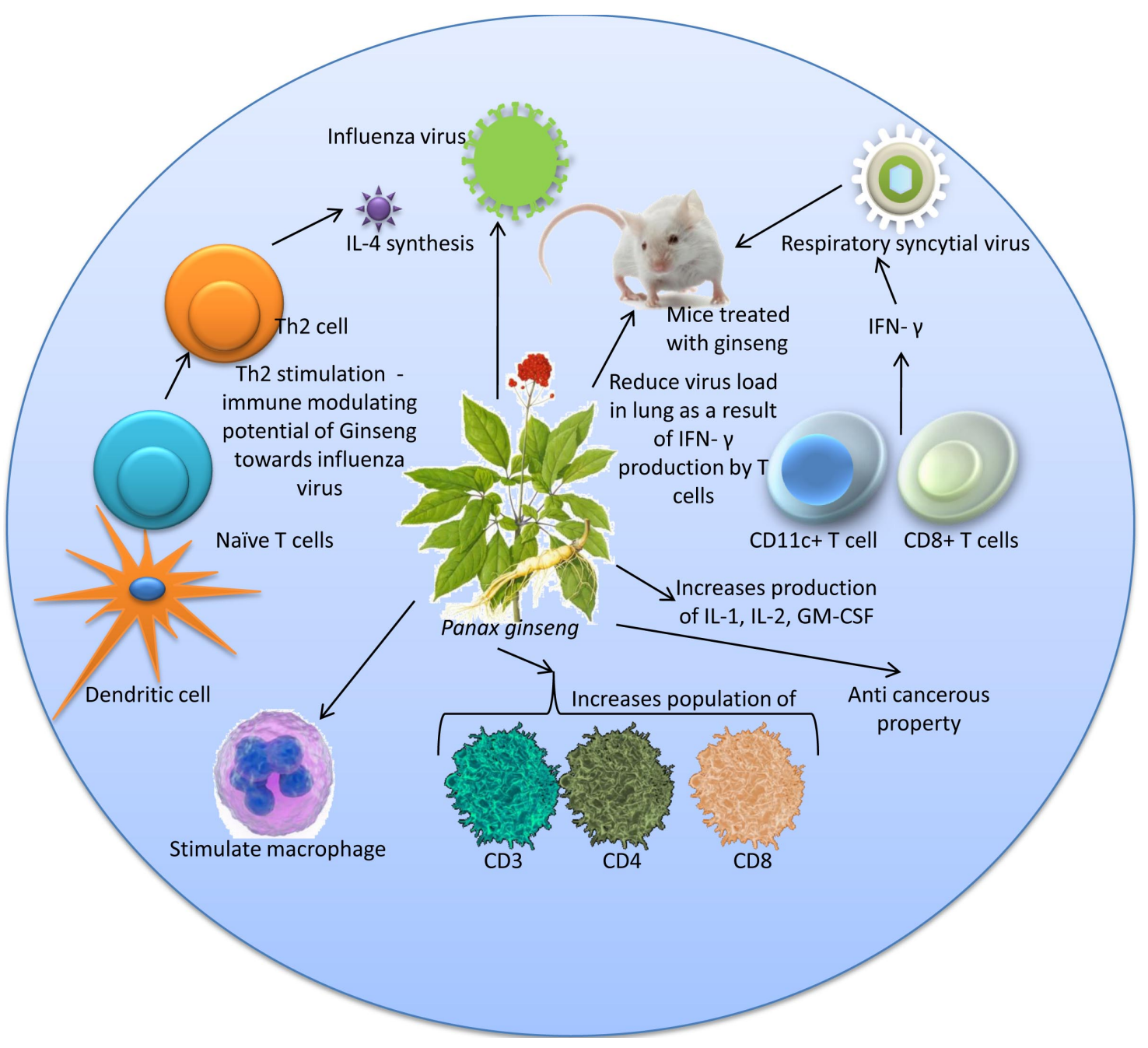

Figure 15. Immunomodulatory activities of Panax ginseng

\section{Thyme (Thymus vulgaris)}

Thyme is mostly grown in Mediterranean regions and has received increasing attention because of its antibacterial and antioxidant properties. The presence of carvacrol and thymol (Fig. 16) in its essential oil is responsible for antioxidant properties [480,481]. Also, it has a broad range of anti-bacterial activity against different pathogenic and microbes [482]. Furthermore, these phenolic constituents show considerable anti-fungicidal and antimicrobial properties [483]. Pharmacological assessment of thymol after oral supplementation in mice indicates that thymol sulfate and thymol glucuronide could be detected in urine whereas thymol sulfate detected in plasma for up to $38 \mathrm{~h}$ followed by its renal elimination completed within $24 \mathrm{~h}$. An average elimination half-life of thymol was found to be $10.2 \mathrm{~h}$, and thymol could be found only in biotransformed forms in plasma or urine [484]. 
Thymol is currently in use against oral bacteria [485]. In earlier studies, Allen et al. [486], Denil et al. [487] and Cross et al. [488] confirmed therapeutic influences of thyme in the production of poultry. Abd El-Hack and Alagawany [489] claimed that immunoglobulin G and A were significantly $(P<0.05)$ affected in laying hens fed diets enriched with thyme compared with the control group. Authors explained that serum IgG and IgA were raised in birds fed a diet supplemented with $3 \mathrm{~g} / \mathrm{kg}$ of thyme ( $P<$ 0.001 and $P<0.042$, respectively), in contrast with the other dietary groups. Contrarily, Karimi [490] noticed that medicinal herbs added to layer diets did not statistically affect IgM compared with the control group. Abd El-Hack and Alagawany [489] added that supplementing thyme to layer diets may enhance the immune system due to the elevation in immunoglobulin concentrations (IgG, $\operatorname{IgA}$, and $\operatorname{IgM}$ ) in layers given diets with thyme versus hens fed a control diet. It is likely that a lower amount of natural phytogenic feed additives may be needed to activate the humoral immune response. Previous investigations revealed that thyme and its components could activate immune functions such as phagocytosis, lymphocyte proliferation, hemoglobin levels and red and white blood cell counts [491]. As a medicinal plant, Thymus vulgaris need to be focused with particular attention in pharmacological research on developing novel drug candidates of practical and therapeutic importance.

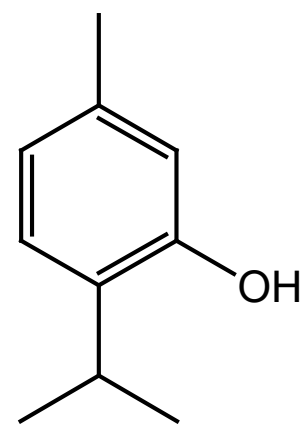

A

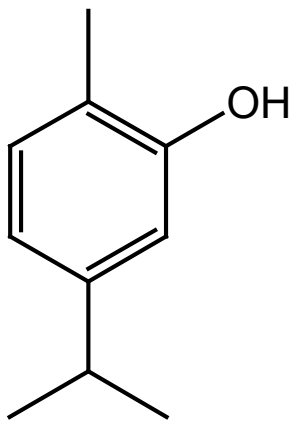

B

Figure 16: A. Thymol and B. Carvacrol: The major bioactive components of Thyme

\section{Terminalia arjuna (Arjun)}

With many local names (Arjuna, Koha, Kahu, Arjan, and White Marudah), this plant is commonly available and amply cited in Ayurveda as a cardiac tonic [492, 493]. Tannins, arjunic acid, arjunantin and stilbesterol are the major components in Terminalia arjuna extract [494, 495]. Bark decoction of arjun believed to be most effective with sugarcane juice. It has also been used for immunopotentiation in animals wound treatment and fracture. Anti-tumor function, against Dalton's lymphoma ascites tumor cells, of extracts of this herb, was recently studied [496]. The value of closely related plant Terminalia puniculata in the treatment of chronic inflammation and arthritis in mice is observed [497]. Hemalatha et al. [498] reviewed multiple therapeutic functions of arjunolic acid extracted from T. arjuna. Kapoor et al. [499] studied the cardio-protective impacts of T. arjuna on immuno-inflammatory markers in coronary artery disease, and they found that the administration of this plant (500 $\mathrm{mg}$ twice a day) improved the immune functions. T. arjuna is a proven anthelminthic; its aqueous extract showed higher potency in mediating anthelmintic activity than that of the methanolic extract [500]. Several studies based on the methanolic extract of $T$. arjuna bark portion revealed a higher microbial activity against Gram-negative bacteria than that of Gram-positive ones, along with promising antioxidant effect [501]. The alcoholic and aqueous bark extracts of T. arjuna containing bio-active molecules e.g. arjunic acid, arjunetin and arjungenin have reported with an inhibitory effect on liver microsomal enzymes like CYP3A4, CYP2D6, CYP2D9, etc. thereby suggesting its potential for inducing inter-drug interactions [502]. Various trials on T. arjuna reported its effectiveness as an anti-ischemic drug with the potential to reduce atherogenic lipid. However, incessant pharmacological trials and evaluations regarding its molecular actions, drug interactions, and side-effects are still demanded [503]. Further phytochemical screenings of the plant and its extract need to be applied for revealing the probable presence of active principles as future drug candidates. 


\section{Sambucus nigra (Elderberry)}

Elderberries are popular among different traditional medicines. The extracts are used for multiple purposes as diuretic, diaphoretic, astringent and laxatives. Syrup from the berries is used to treat cold and upper respiratory infections. Active constituents include flavonoids, rutin, anthocyanin and mucopolysaccharides [504]. Anthocyanins are the potent flavonoid antioxidants found in the plant that is absorbed in their glycosylated forms through the gut, reaching a maximum concentration by $1 \mathrm{~h}$, and subsequent decay immediately. Elimination of plasma anthocyanins follows first-order kinetics as evidenced by the two major anthocyanins cyanidin-3-glucoside and cyanidin-3-sambubioside, anthocyanin compounds which can be detected in urine $[505,506]$. Antiviral effects of Sambucus nigra extracts are well observed against influenza, herpes simplex virus 1 and HIV [507-509]. Immunomodulatory activity encompasses activation and mobilization of phagocytes to inflamed areas [510]. The polyphenolic compounds isolated from Sambucus nigral lowered the inflammatory status and enhanced the specific and non-specific immune defenses in insulin-deficiency diabetes [511].

\section{Bauhinia variegata Linn. (Leguminosae)}

Bauhinia variegate Linn. grows in extensive plantations and moist wasteland. It is widely cultivated in India and also abundantly exists in forestlands of central India. As stated by Ayurveda Bauhinia variegata Linn. is used as a tonic for the treatment of leprosy, blood impurities, liver, wounds, menorrhagia, ulcer, tuberculosis and asthma [512-514]. An ayurvedic remedy indicated for increasing WBC, herbal tonic kanchanar guggul, contains the bark powder of this plant as a major ingredient. Steriods, flavonoids, tannins, saponin and alkaloids were confirmed present in the phytochemical screening of Bauhinia variegate Linn. [515-517]. Besides, vitamin C, quercetin, flavanone, lupeol, $\beta$ sitosterol, and kaempferol have also been reported in other studies to be found in the ethanolic extract of Bauhinia variegata. Moreover, its antibacterial, antifungal antiulcer and antitumor actions are observed in some studies $[514,518]$. Immunomodulatory effect of $B$. variegate was evidenced by the potential of ethanolic extract of its stem bark extract which enhanced both primary and secondary antibody responses. Also, it can improve the natural immune compounds like neutrophil function and phagocytosis [519].

\section{Eleutherococcus senticosus (Acanthopanax senticosus)}

Eleutherococcus senticosus previously known as Siberian ginseng and commonly called ciwujia is indigenous to far Eastern regions of Russia, Northern areas of Korea, Japan and China [48, 107, 520]. The plant extract is popularly known as adaptogen as it helps the body to adapt physical and emotional stress. Adaptogen increases energy and resistance to many diseases, counteract stress and produce a normalizing effect [107, 521]. Mainly the root contains chemically distinct glycosides known as eleutherosides A-M with higher concentration which are the active ingredients [522]. Eleutherococcus decreases depletion of adrenal vitamin $\mathrm{C}$ level in stressed rats as a result of its effect on decreasing adrenal hypertrophy [523]. The animals treated with aqueous extract of the stem bark of this plant were found with increased swimming time to exhaustion [524]. Also, its extracts enhanced the cytostatic activity of NK cells by 200 percents [525]. It is also found to modulate cytokine expression and their activity [526]. In rats with induces cerebral artery occlusion Eleutherococcus noticeably inhibited COX-2 expression and reduced cerebral ischemia [527]. Defense against induced fulminant hepatic failure via antioxidant or apoptosis as possible mechanisms [528] and prevention of bone resorption during experimental, steroid induced osteoporosis, are among additional biological activities of Eleutherococcus [529]. Recommended doses of the herb include 2-3 g powdered root one daily or in the form of tea having 2-3 $\mathrm{g}$ in a cup of boiling water which is taken twice a day [107].

\section{Hibiscus sabdariffa (Hibiscus)}

This medicinal shrub belongs to a tropical part of Africa and Asia and mostly cultivated in the hotter parts of India. Commonly called as 'rosella,' all-ground parts of this plant are high in bioflavonoids and vitamin $\mathrm{C}$ and indicated for bruising, mild cold and swelling [530]. Calyx infusion of $H$. sabdariffa decreased mitogen-induced blastogenesis of lymphocytes in normal subjects which may help to overcome hypersensitive conditions [531]. Extracts from the dried calyx containing anthocyanins proved to be valuable in treating hemotoxicity and oxidative injury by 2-4-dinitrophenyl hydrazine in rabbits 
[532]. Aqueous extracts promote the production of IL-6 and IL-8 decreases synthesis of monocyte chemoattractant protein-1 (MCP-1) in peripheral mononuclear cells indicating its value in the treatment of chronic inflammatory diseases [533]. Traditionally, Hibiscus sabdariffa have beneficial therapeutic effects likewise lowering blood pressure with minimal side effects. Its protective efficacy on human vasculature and inhibition of angiotensin converting enzyme has been demonstrated in the laboratory [534]. Evaluation of the chemical nature of its stem and leaves indicates the presence of phenolic compounds such as tannins and flavonoids that impart the antioxidant properties to the plant [535].

\section{Watercress (Nasturtium nasturtium)}

Watercress or Nasturtium is a useful medical plant comprising various bioactive substances. Nasturtium nasturtium is usually cultivated for cookery, because of its pungent leaves. It is especially used in soups, garnishes, and salads. Watercress leaves are used as normally processed vegetables or consumed as a green salad. Watercress is one among the most prescribed herbal medications for treating asthma [536], diabetes [537], immune depression [538] oxidative stress [539] and scorch [540]. It is a fine source of vitamins and a good detoxifying medicinal agent. It contains a significant quantity of vitamins $B_{1}, B_{2}, C$, provitamin A, glucosinolates (Fig. 17), iron, iodine, protein and especially sulfur and calcium compounds, which are responsible for its pleasant odor while enhancing its nutritional values [541-543]. Concerning various research findings published, watercress is an essential supply of glucosinolates. Being hydrolyzed glucosinolates are capable of producing phenethyl isothiocyanate. These compounds (isothiocyanates) inhibits phase I enzymes like CYP450 and as a consequence could avoid carcinogen activation [544] and improves excretion of potential carcinogens through activating phase II enzymes like glutathione-S-transferase, glucuronosyltransferases, quinine reductase and oxidoreductase [545, 546]. Furthermore, watercress extract support fibrosis inhibition [540], inflammation [536], and the CYP459 activity [541]. Watercress also inhibits lipid peroxidation as a consequence of its antioxidant action [545, 546]. Asadi et al. [547] reported that using $1 \%$ of watercress extract in fish diets may lead to improved fish immunity.

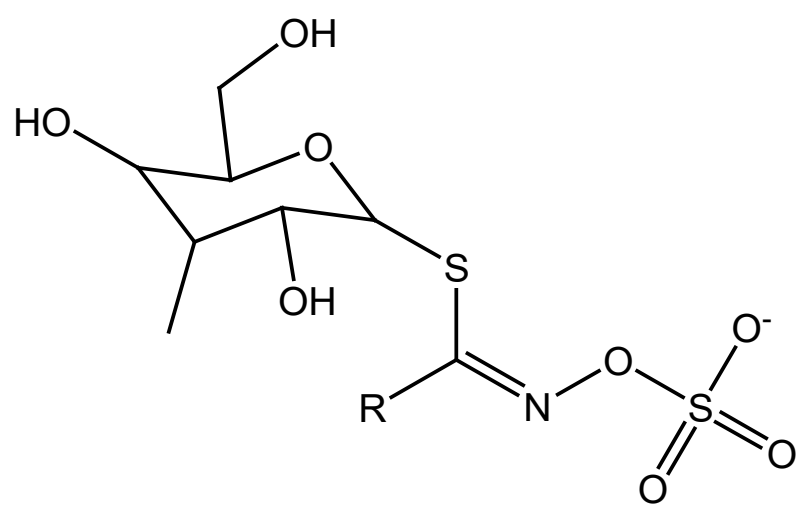

Figure 17: General structure of glucosinolates which are responsible for producing isothiocyanates

\section{Nyctanthes arbor-tristis (Harsinghar- Oleaceae):}

This plant also called as Night jasmine or Coral jasmine, is a native of South and Southeast Asia. Its leaves, flowers, and seeds are extensively used in traditional herbal therapy for conditions like arthritis, scurvy, and malaria. It possesses multiple functions such as antifungal, antileishmanial, immunomodulatory and hepatoprotective activities [548]. Its phytochemistry revealed several bioactive chemicals that include phytosterols, tannins, flavonoids, phenolics, saponins and glycosides [549]. Immunomodulatory and antileishmanial activities may be attributed to the presence of glycosides especially iridoid and phenylpropanoid glycosides [550]. Several in-vitro experiments have proved its natural antioxidant activity which is due to the presence of acetone-soluble fraction as evidenced by hydroxyl, superoxide radicals and hydrogen peroxide scavenging assays [551].

Puri et al. [548] employed ethanolic extracts of the leaves, seeds, and flowers to demonstrate the immune enhancing property of $N$. arbortristis. The results indicated increased humoral and DTH response to sheep RBC and macrophage migration. Oral administration of iridoid glycosides of this plant 
to Swiss albino mice demonstrated protection against Candida albicans by stimulating humoral immune response and macrophage activity [552]. Its involvement in various conditions such as the antiinflammatory, antinociceptive and antipyretic medication is due to the water soluble fraction. $N$. arbortristis's root and ethanolic seed extracts were demonstrated with satisfactory immunomodulatory properties against systemic candidiasis in mice. There are also reports about the antiallergic and sedative nature of its alcoholic extract [549].

\section{Terminalia chebula (Combretaceae)}

T. chebula is a plant indigenous to Southeast Asia and belongs to the family of Combretaceae [553]. It is named Harad in Hindi and known as black myrobalan in English. The dry ripe fruit of T. chebula is widely used as adjuvant formulation to Triphala and Menosan or alone in traditional Indian and Asian medicine [554]. The mature/immature fruits are used as an antioxidant due to the presence of natural phenolics e.g., gallic acid. A range of pharmacological activities have been reported in association with T. chebula such as antiviral [555], antibacterial [556], antidiabetic, renoprotective [557], radioprotective [558], anticancer [559], antioxidant and adaptogenic activity [560, 561]. Chemical constituents such as flavonoids, tannins, sterols, fructose, amino acids, resins and fixed oils have been found in Terminalia species. Also, anthraquinones, chebulagic acid, chebulinic acid, ellagic acid, gallic and ethaedioc acid, terpinenes and terpineols and 4,2,4-chebulyl- $\beta$-D-glucopyranose have been identified in this species [54, 556, 559]. The result of Vaibhav et al. [554] and Rubab and Ali [562] demonstrated the immunomodulatory activity of $T$. chebula. The antibacterial property of the plant is attributed to the heat stable compounds with possible therapeutic potential [563].

\section{Boerhavia diffusa (Erva Tostao - Nynctaginaceae)}

Boerhavia diffusa, commonly called Punarnava or Red Hogweed, is a herbaceous member belonging to the Nynctaginaceae family. It is extensively used by indigenous people especially for the Ayurvedic and Unani practice in the Indian subcontinent [564]. Various parts of the plant are mainly applied to get relief from conditions like body pain and diabetes mellitus. It was found as a regulator of the blood sugar level which underlines its application in diabetes mellitus [565]. It also possesses certain other functionalities such as diuresis, antifibrinolytic, anti-convulsion, anti-inflammatory and hepatoprotection [566-568]. The biologically active compounds in $B$. diffusaare have been found to be sterols and alkaloids. For example, ursolic acid, myricyl alcohol, myristic acid, hypoxanthine-9-L-arabinofuranoside and punarnavine 1 and 2 are the important components. These agents are the major factors for imparting antidiabetic long with antioxidant activity to $B$. diffusa. It exhibits antioxidant function with its defending effect on lipid peroxidation at the same time by promoting the cellular based antioxidant defense [567]. Pharmacodynamics combining the application of $B$. diffusa root extract along with its interaction with anti-ulcerative drugs like Omeprazole showed a syngeneic influence, resulting in an efficient reduction in the inflammation and subsequent remission of gastric ulcer in a rat model. It exerts the anti-inflammation mediated gastric ulcer protection through the suppression of both gastric acid secretion and gastric action [569].

Mungantiwarn et al. [570] and Mehrotra et al. [571] studied the immunomodulatory effect of $B$. diffusa alkaloidal fraction along with an ethanolic extract from its root for immunomodulation. In-vitro studies revealed that the ethanolic extracts of $B$. diffusa could inhibit NK cell cytotoxicity in human, NO production from mouse macrophages, IL-2 and TNF- $\alpha$ secretion by human PBMCs. Both in-vitro and invivo studies by Manu and Kuttan [572] demonstrated the action of Punarnavine, a glycoside from $B$. diffusa, in enhancing the proliferation of splenocytes, thymocytes, and bone marrow haematopoietic cells. The role of Punarnavine in immunomodulation is evident from the significant reduction in the levels of LPS induced pro-inflammatory cytokines. Its antibacterial action against pathogens like Gonococus, Bacillus, Pseudomonas, Salmonella and Staphylococus as well as antiviral action against viral plant pathogen were also reported by various scientists [573, 574].

Certain tribal population finds the therapeutic potential of this herb in the cure of conditions like leukorrhea, and rheumatism. While some others used for seminal abnormalities and also as a blood pressure regulator. It is observed as a good anthelminthic especially in the tropical countries that too among the indigenous therapy. The hepatoprotective effect of $B$. diffusa has been subjected to different experimental trials especially in the case of liver damage induced by high doses of analgesics like 
paracetamol. In analgesic-induced liver damage, the serum level of liver-specific enzymes like ALT and AST found to be elevated. The primary reason for the protective role of $B$. diffusa in these types of hepatic abnormalities may be its demonstrated action in preventing the rise in enzyme activity [575-579].

\section{Yucca (Yucca schidigera)}

Yucca is widely grown in the deserts and commercially used as a source of saponin, especially steroidal saponins. Furthermore, yucca is an excellent source of several polyphenolic and phytochemicals compounds e.g. resveratrol, yuccaols A, B, C, D, and E, respectively [580]. Yucca or its extract is used as a natural supplement and flavor enhancer in the beverage and food industries. In livestock industry, it is used as a phytogenic feed additive [21]. Several studies showed various biological effects and protective impacts of yucca, involving antioxidant, anti-inflammatory, immunomodulatory, antibacterial and health improving properties [581, 582]. It contains very high amounts of phenolic molecules and saponins with antioxidant activity. Alagawany et al. [21] reported that nutritional supplements of yucca showed beneficial action on IgG and albumin which are comparable to those reported by Ashour et al. [582]. Alagawany et al. [21] theorized that supplementing layer nutrition with this herb linearly and quadratically $(\mathrm{P}<0.001)$ increases IgG level. Authors concluded that yucca supplementation to layer diets could improve the immune system through increasing immunoglobulin levels comparing to the control diet. Alagawany's study suggested that natural phytogenic additives in feeds at a lower dose may be needed to arouse and activate a humoral immune response in poultry [21]. Yucca saponins can be of antibacterial nature and regulate ruminal fermentation through the inhibitory/suppressive action on ruminal protozoa and selective destruction of some enteropathogenic bacteria [581].

\section{Goldenseal (Hydrastis canadensis L.)}

Goldenseal is indigenous to the American continent and belongs to the Ranunculaceae family. It is exploited as a medicinal herb due to its relatively high concentration of alkaloids [583]. This herb is potentially considered as a potent antibacterial agent, particularly when applied in the form of crude extracts as well as isolated compounds $[584,585]$. It has been demonstrated that the antibacterial property is attributed mainly by the alkaloid named berberine which is proven by other in-vitro and clinical trials [586]. The bioactive compounds in goldenseal are mainly comprise of isoquinoline alkaloids. Potential examples include berberine, canadine, and beta-hydrastine (Fig. 18). The antimicrobial action of berberine may also be due to the synergistic effect from other alkaloids [583, 587]. Goldenseal was proven to compact efficiently the proliferation of pathogenic agents especially those with Staphylococcal, Streptococcal, Escherichia and Pseudomonas origin [584]. Certain studies conducted for the analysis of these constituting alkaloids indicate that they may be exerting their therapeutic efficacy through adrenergic and adenosine receptors [588]. Thus Goldenseal can be practiced for herbal induced immunopotentiation mediated through the presence of its alkaloids especially berberine.<smiles></smiles>

A<smiles>COc1ccc2c(c1OC)C[NH+]1CCc3cc4c(cc3C1C2)OCO4</smiles>

B<smiles>COc1ccc2c(c1OC)C(=O)OC2c1c2c(cc3c1OCO3)CCN(C)C2</smiles>

C

Figure 18: A. berberine, B. canadine, and C. beta-hydrastine; the pharmacologically active components of Hydrastis canadensis L. 


\section{Marigold (Calendula officinalis L., Asteraceae)}

This is a popular herbal remedy in both eastern and western parts of the world especially as cosmetic and at the same time with the anti-inflammatory property. In the Indian subcontinent, this plant is mainly employed as a remedy for psoriasis and other skin conditions like acne and wound [589]. Different active agents have been isolated from the plant involving triterpene saponins, alcohols, carotenoids, coumarins, fatty acid esters, flavonoids, essential oils, hydrocarbons and fatty acids [590, 591]. Among these active agents, the anti-inflammatory properties have been imparted by fatty acid esters of triterpenoid nature which has been validated by various in-vivo experiments [592]. The essential oils from C. officinalis have found with application in soothing the central nervous system along with healing properties [593]. Aswagandha root powder According to the study by Roopashree et al. [589], it is indicated that the aqueous extract of marigold exhibits antibacterial action against Staphylococcus aureus. Immunostimulating property of $C$. officinalis is attributed to the presence of its polysaccharide fraction and had been validated by invitro granulocyte tests, showing that the polysaccharide $3^{\text {rd }}$ fraction possesses highest (54-100\%) phagocytic activity [594]. Toxicological evaluations conducted in-vivo in mice and rats suggest its hydroethanolic extract being non-toxic to even in some higher oral dosages but shown to elevate the blood urea nitrogen and alanine aminotransferase levels, indicating liver overloading [594, 595].

\section{Herbal medicines: for counteracting temperature stress-induced immunosuppression in livestock and poultry}

The mammalian immune system can be sensitized not only by microbial PAMPs but also due to endogenous sensory molecules released following multiple stresses imposed cellular damage. Among abiotic stresses, temperature stress seems to be the most common impediment affecting animal production systems. Due to this perpetual temperature menace, the immune system of tropical livestock is always sensitized or compromised in their niche [596]. Immune stimulation in animals is characterized by the production of inflammatory cytokines, acute phase proteins, fever, inappetence, etc. The activated immune system may disturb the homeostatic pathways, and consequently reduced the growth [597]. Hence, the production potential of livestock and poultry population, native to arid and semiarid tropical countries, cannot be tapped fully due to impinging elevated ambient temperature. Production can be augmented by counteracting these hurdles through the supplementation of dietary immunomodulatory agents. Among them, phytochemicals and herbal immunomodulatory agents can occupy a centric position since they are cost-effective, with no or minimal side-effects, user-friendly and easily available, etc. [82]. Since heat stress can modulate the immune system towards anti-inflammatory and immune compromised status, immune-stimulatory supplements are of high impact in this condition, among which herbal preparations can have a significant place $[598,599]$. In heat stressed and immune compromised livestock, Endogenous Heat Shock Proteins (HSPs) act as molecular chaperones and protect the cell from heat-induced damage. Several compounds from plant origin are proven as endogenous HSP inducers or co-inducers, suggesting the possibility of exploring them as anti-inflammatory supplements [600].

Many herbs have been or being evaluated for heat stress through immunomodulation. It was reported that Angelica gigas Nakai, a traditional Korean herb from Umbelliferae family could ameliorate the heat stress-induced damages in murine splenocytes [601]. This herb has been conventionally used in cardiovascular and inflammatory disorders [10,602]. Recent findings by Lumbera et al. [601] suggest its ability to enhance the expression of molecular chaperones (Heat shock proteins or HSPs) and to induce Th2 mediated cell-mediated immunity. Curcumin, the active principle in Curcuma longa (turmeric) is a co-inducer of stress-related proteins and found to mediate anti-inflammation by inhibiting the proinflammatory transcription factor NF- $\kappa \mathrm{B}$ [603]. Upon heat stress exposure, it can increase the expression of heat stress chaperones like HSP27, $\alpha \mathrm{B}$ crystallin, and HSP70. Its arthritis and joint erosion inhibitory potentialities have also been reported in a dose-dependent manner in the $\mathrm{T}$ cell mediated approach using an experimental model of streptococcal cell wall-induced arthritis [604]. A study conducted in broilers for assessing the impact of Tulsi (O. sanctum) upon cortisol production and immune response revealed that supplementation of Tulsi could limit the serum cortisol level and augment immunity [605]. Herbal medicine constituents such as celastrol, a triterpene compound from Tripterygium wilfordii (Thunder god vine), paeoniflorin, derived from Paeonia lactiflora and glycyrrhizin from Glycyrrhiza glabra (liquorice) were proven to be of heat shock protein co-inducers, suggesting their potential role in 
heat stress amelioration [600,606, 607]. The pharmacological effects of Ginseng in limiting the heat stress-induced cellular damage and subsequent inflammatory changes in murine models were reported by Yoon et al. [608]. Sisymbrium irio, a member of Brassicaceae family widely used in middle-east countries for inflammatory conditions, has proven its heat stress alleviation effect on Awassi sheep [609].

Herbal formulations either using an individual of mix plants have been evaluated and used extensively in livestock production system. Supplementation of a specific combination of cinnamon, turmeric, and ginger along with ascorbic acid through diet had a favorable response to growth indices, hematological parameters and antioxidant status of heat-stressed broiler chicks [330]. Traditional Chinese Medicines constitutes a major firm in herbal medicine practice with their unique combinations of herbal preparations [610]. Various scientific studies in Chinese herbal medicines revealed that the biologically active constituents from Cortex Phellodendri, Rhizoma Atractylodes, Agastache rugosa and Gypsum Fibrosum could prevent animals from heat stress induced diseases [611]. Aurantii Nobilis Pericarpium (Hesperidin) and Magnolia officinalis (Magnolol) are widely used in Chinese medicines for their antioxidant and immune stimulating properties which can be practiced in heat stress management $[612,613]$. Heat stress amelioration in broiler birds was evidenced by Ashwagandha root powder as well as extract supplementation by enhanced body weight, serum antioxidant concentration and improved immune status [82].

\section{Commercial herbal preparations claiming as immune boosters}

Herbal remedies targeted for commercial distribution are mainly manufactured as Nutraceutical formulations. Herbs or their active compounds (phytochemicals) in combination with other chemical or non-chemical constituents are presently occupied an important position with some particular emphasis in nutraceutical industry, worldwide [614]. Some of the popular herbal drugs available commercially are listed below and presented in Table 1.

ImmuPlus ${ }^{\mathbf{R}}$ : A polyherbal preparation of four herbs including Ocimum sanctum, Tinospora cordifolia, Emblica officinali and Withania somnifera. Its immunomodulatory activity is widely studied in the veterinary field. It increases antibody titer in the dog, poultry, and mice while stimulating blastogenic capacity of $\mathrm{T}$ and $\mathrm{B}$ cells. Its immunopotentiation activity in pups is observed in 'conjunction with parvovirus and rabies vaccine [127]. Similar results were also demonstrated with infectious bursal disease and Newcastle disease vaccine in chickens [128]. Results of Priyadarshini et al. [615] confirmed the ability of ImmuPlus to enhance growth and improve the immune and inflammatory response of common carp by administration through diet. Moreover, effective immunomodulatory effects of ImmuPlus were observed in broiler chickens [616].

Immu-21 $^{\text {R: }}$ Its constituents are identical to Immuplus $^{\mathrm{R}}$. It has shown protective effects against mutagenicity in mice induced by cyclophosphamide [617]. Phagocytic activity of neutrophils and peritoneal macrophages was observed to increase with this preparation. It has shown improved production of colony forming units of bone marrow mononuclear cells and GM-CSF in mice [618].

Dalneen $^{\mathrm{R}}$ : It is a proprietary herbal preparation containing neem oil indicated for the treatment of bovine udder ulcer. In one of the clinical study, $6 \mathrm{~cm}$ chronic ulcer lesions on udder in 10 Holstein Friesen cows were treated with neem oil $\left(\right.$ Dalneen $\left.^{\mathrm{R}}\right)$ and complete healing of the lesions occurred in 7 days of treatment [619].

Echinacea liquid $^{\mathrm{R}}$ : Another herbal preparation mainly made up of extracts from Echinacea augastifolia and used in the equines at the rate of 40 to $50 \mathrm{ml}$ per day. It is proposed to stimulate the immune system thus improve the body's resistance to both bacterial and viral infectives. Echinacea is also used against many other infections including vaginal yeast infections, urinary tract infections, herpes, HIV/AIDS, human papillomavirus (HPV), bloodstream infections (septicemia), tonsillitis, syphilis, streptococcus infections, typhoid, malaria, ear infection, warts, swine flu and throat and nose infections called diphtheria [620].

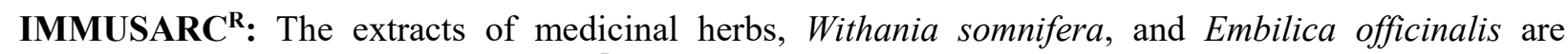
combined and presented as Immusarc ${ }^{\mathrm{R}}$. It is believed to help for the optimum function of immune system. 
ROSEHIP $^{\mathrm{R}}$ : Proprietary herbal preparation with high vitamin $\mathrm{C}$ contents claimed as a boost for natural body defense and used in large animal practices.

Sambucol $^{\mathbf{R}}$ : It is syrup with a standardized extract of elderberry amounting to $38 \%$ of its content. Infectivity of HIV, Herpes simplex virus type 1 and influenza viruses are observed to neutralize and reduce with sambucol preparations [507, 508, 509]. This syrup also found to have immunomodulatory functions e.g. increased production of different cytokines (TNF- $\alpha$, IL1 $\beta$, IL-6) and activation of phagocytes [510].

uMakhonya $^{\mathbf{R}}$ : It is one of the widely used commercial immune boosters which use THP-1 monocyte cells. This traditional commercial preparation was reported9- to induce dose-dependent cytotoxicity with high doses significantly $(\mathrm{P}<0.05)$ cytotoxic to monocytes $\left(\mathrm{IC}_{50}\right.$ of 100.08 and $107.68 \mu \mathrm{g} / \mathrm{mL}$ for normal and LPS stimulated THP-1 cells respectively) comparing to untreated cells [621].

Cordyceps $^{\mathrm{R}}$ : Is the composite of fungus which grows on the larva of insects. Till now, more than 350 species of Cordyceps-related have been observed worldwide subject to the fungus and insect host basis. Since the year of 1964, only Cordyceps sinensis has been officially recorded as an herbal drug in Chinese pharmacopeia. C. sinensis, and known as Dongchongxiacao (winter-worm summer-grass) in Chinese, is one of the most famous traditional Chinese medicines and medicinal mushrooms [622].

E-care Se Herbal: This is a poultry oriented immune booster supplied by Virbac India Pvt. Ltd., amalgamating the potentials of Ocimum sanctum (tulsi) extract along with Vitamin $\mathrm{C}$ and Selenium in immune stimulation. This formulation has been evaluated in-vivo in counteracting the immune suppression mediated by chicken infectious anemia virus (CIAV). It was also found to be effective in particular through the modulation of cell-mediated immune response as well as reducing viral load in birds $[623,624]$.

Tinofend: This is a proven immune modulating medicine from Life Extension, prepared from the standardized active constituents of Tinospora extract. Tinofend stabilizes the overall cellular interactions and responses for a balanced immunity mainly by enhancing the function of macrophages, regulating the proliferation of eosinophils and by improving neutrophil-mediated innate immunity through its polysaccharides. Effect of Tinofend in promoting immunity is evidenced by a clinical trial on humans by Badar et al. [625] in which they investigated its efficacy in controlling allergic rhinitis through the antiallergic, anti-inflammatory and immunostimulating properties of $T$. cordifolia.

Immon: 'Immon' (Regen Biocorps, Vadodara, Gujrat, India) is a polyherbal formulation comprising of Asparagus adscendens, Moringa oleifera and Picrorhiza kurroa with micro and macro nutrients (amino acids, minerals, vitamins $\mathrm{E}$ and $\mathrm{C}$, nucleotides, spirulina, $\beta$-glucans and probiotics). Immon has been recently found to be an effective immunomodulating agent in reducing the pathogenicity of chicken infectious anemia virus (CIAV) affected birds, ameliorating the depressed immune responses and protecting the adverse effects on growth performances [626].

Mastilep: Mastilep is a topical herbal gel comprising of Eucalyptus globulus - $0.20 \mathrm{~g}$, Glycyrrhiza glabra - $0.20 \mathrm{~g}$, Cedrus deodara - $1.00 \mathrm{~g}$, Curcuma longa - $0.04 \mathrm{~g}$, Paedaria foetida - $0.04 \mathrm{~g}$ and sulphur $-1.00 \mathrm{~g}$ which is reported to possess antibacterial, anti-inflammatory and immunomodulatory properties. This preparation has been used for the treatment of bovine mastitis. Studies indicated that this gel had increased production of cytokines like IL-8 and INF- $\gamma$ [627].

HemoHIM: an Herbal preparation consisting of Cnidium officinale Makino, Angelica gigas Nakai and Paeonia japonica Miyabe was reported to prevent hyperglycemia and also alter the immune response of diabetic mice. Animal experiments showed that this preparation could increase white blood cells count, lymphocytes, and also activated NK cells. Studies using streptozotocin-induced diabetic mice demonstrated that it could prevent hyperglycemia [628].

UMakhonya $^{\circledR}$ : The product comprised of African wormwood, menthol, Psidium guajava, Chondrus crispus and Uncaria tomentosa. A study revealed that this product increased IL-1 $\alpha$, IL-1 $\beta$, IL-6, IL-10, TNF- $\alpha$, and granulocyte-macrophage-colony-stimulating factor in immunosuppressed mice. It is recommended for cleansing the blood and for diabetes, blood pressure, pneumonia, ulcers, and hemorrhoids [629]. 
Table 1: Some of the other commercially available herbal medicines are listed below.

\begin{tabular}{|c|c|c|}
\hline No. & Drug & Description \\
\hline 1. & Herbal Zap & $\begin{array}{l}\text { It is a reviving and luscious preparation made out of some fourteen herbs, } \\
\text { especially Vasaka, Ajowan, Coriander, Long pepper, Black Pepper, Cumin, } \\
\text { Ginger, etc. This medicine is a herbal extract-based proprietary blend, } \\
\text { designed to boost the immune functions along with effective digestive and } \\
\text { respiratory functions. }\end{array}$ \\
\hline 2. & Wellness Formula & $\begin{array}{l}\text { This is a drug with herbal defense complex containing powerful herbal } \\
\text { medicines in combination with antioxidants, vitamins, and minerals, } \\
\text { intended to be effective in boosting immunity under conditions of stress. It } \\
\text { mainly contains the extracts of Andrographis and Propolis along with the } \\
\text { goodness of other plants such as Elderberry fruit, Garlic bulb, Olive Leaf, } \\
\text { Elecampane Root, Citrus Bioflavonoid Complex, Astragalus Root Extract, } \\
\text { Ginger Root Extract, Grape Seed Extract, etc. }\end{array}$ \\
\hline 3. & $\begin{array}{l}\text { Zingiber-Immune } \\
\text { (California } \quad \text { Gold } \\
\text { Nutrition) }\end{array}$ & $\begin{array}{l}\text { This herbal dietary supplement is a wonderful combination of Ginger, } \\
\text { Sambucus, and Echinacea with enhancing influence on the immune system, } \\
\text { along with soothing effects to upper respiratory tract and stomach ailments. }\end{array}$ \\
\hline 4. & $\begin{array}{l}\text { Double } \quad \text { Strength } \\
\text { Silymarin (Now Foods) }\end{array}$ & $\begin{array}{l}\text { This is a vegetarian formula based on Silymarin which is obtained from the } \\
\text { plant Silybummarianum, also known as Milk Thistle, having centuries-long } \\
\text { medicinal tradition. Research investigations indicate that active ingredients } \\
\text { in Silymarin can support hepatic and cardiac functions. This also includes } \\
\text { Artichoke \& Dandelion for additional support. }\end{array}$ \\
\hline 5. & $\begin{array}{l}\text { AmritKalp } \\
\text { Ayurveda) }\end{array}$ & $\begin{array}{l}\text { This drug which has been used by a large proportion of consumers enhances } \\
\text { the vital capacity, helps in building up body muscles and altogether it } \\
\text { rejuvenates the body. }\end{array}$ \\
\hline 6. & Tulsihills (Herbal Hills) & $\begin{array}{l}\text { It is a popular herbal antioxidant and immune booster made out of natural } \\
\text { Tulsi extract. It is prepared from standardized Tulsi extract which contains } \\
7 \% \text { Tannins and } 0.5 \% \text { Ursolic Acid. This can be a potent adaptogenic drug } \\
\text { also. }\end{array}$ \\
\hline 7. & $\begin{array}{l}\text { Gericaps Active (Health } \\
\text { Aid) }\end{array}$ & $\begin{array}{l}\text { This is a highly potent capsule formulation from Ginseng and Ginkgo biloba } \\
\text { along with the uniquely balanced combination of essential vitamins, } \\
\text { minerals. This capsule is intended for revitalizing the body by boosting } \\
\text { energy levels and provides an overall well-being. }\end{array}$ \\
\hline 8. & Imunohills (Herbal Hills) & $\begin{array}{l}\text { This is a classic amalgamation of immune enhancing herbs like Amla } \\
\text { (Emblicaofficinalis), Guduchi (Tinosporacordifolia) and Gokshura } \\
\text { (Tribulusterrestris) because of which this drug can modulate cellular as well } \\
\text { as humoral components of immunity to compact infections and stresses. }\end{array}$ \\
\hline 9. & Paractin (HP Lifescience) & $\begin{array}{l}\text { Paractin is a patented medicinal preparation (US Patent No: 8,084,495 B2) } \\
\text { from the extract of AndrographisPaniculata (King of bitters) and has been } \\
\text { standardized to Andrographolide, 14-deoxyandrographolide, and } \\
\text { Neoandrographolide. The drug is efficient in ameliorating chronic } \\
\text { inflammations, joint problems and pain stimuli by its blocking action over } \\
\text { inflammatory pathways. }\end{array}$ \\
\hline 10. & $\begin{array}{l}\text { Mastilep } \\
\text { limited) }\end{array}$ & $\begin{array}{l}\text { Herbal gel preparation is possessing anti-inflammatory, antibacterial and } \\
\text { immunomodulatory properties recommended for treatment of bovine } \\
\text { mastitis. }\end{array}$ \\
\hline 11. & $\begin{array}{l}\text { HemoHIM (Kolmar BNH } \\
\text { Co. Ltd. (SBT) }\end{array}$ & $\begin{array}{l}\text { Reported to increase the number of immunocytes or improve their functions } \\
\text { and aids to destroy harmful pathogens and distorted cells, to reduce } \\
\text { unwanted immune reactions. }\end{array}$ \\
\hline
\end{tabular}




\begin{tabular}{|l|l|l|}
\hline 12. & $\begin{array}{l}\text { uMakhonya }{ }^{\circledR}- \\
{[\text { UMakhonya Natural }} \\
\text { Health Products (Pty) Ltd } \\
\text { (Pinetown, South Africa) }]\end{array}$ & $\begin{array}{l}\text { Reported to increase IL-1 } \alpha, \text { IL-1 } \beta, \text { IL-6, IL-10, TNF- } \alpha, \text { and granulocyte- } \\
\text { macrophage-colony-stimulating factor level in immunosuppressed mice. }\end{array}$ \\
\hline
\end{tabular}

\section{Technically Scientific Support and Future Perspectives}

In recent years, with ever increasing scientific knowledge, social awareness, pharmacokinetic and pharmacodynamics studies, and phytochemicals along with a wider spectrum of clinical and toxicological based analytical validation, the use of herbs became more popular and acceptable worldwide as medicinal items. Moreover, as compared to the chemical-based synthetic formulations, natural herbs and naturally occurring plant-derived metabolites are being preferred owing to their easy accessibility and fewer side effects. Also, due to the drug failures, ever increasing drug resistivity and many side-effects of chemical formulations, scientists around the globe, are redirecting their research interests for the safer and natural alternatives. As a new era of treatment and prophylaxis for safeguarding human and animal health [630, 631], the recent advances in the biotechnological and molecular tools in the field of herbal or phytomedicine have a noteworthy solution to this problematic issue with the following objectives: (1) to explore herbal wealth, (2) to elucidate structural and chemical mechanism of action or active principles, (3) to investigate drug receptor screening, (4) to identify interactions between large and small phytomolecules, and (5) to isolate value-added active compounds, etc.

A wider spectrum of plant-derived secondary products may emulate the endogenous metabolites, hormones, ligands and signal transduction molecules during the mechanism of action [632]. Evidently, some secondary plant products may resemble with endogenous metabolites, and as noted by Wink [633], the development of structural similarity between secondary plant products and the endogenous substances of other organisms could be termed "evolutionary molecular modeling." The phytochemicals obtained from the plants can also be used as adjuvants for enhancing the immunity of the host against the vaccine. However, the exploration of novel plant-based natural sources from a range of unexplored areas will provide a new avenue to investigate new or analogous molecules with higher activity and lesser toxicity [634]. In this context, with an aim to achieve an optimal yield, many new highly automated techniques are needed at nano- level for screening, separation or isolation, purification, physiochemical and structural characterization of components from crude plant extracts. Thankfully, the recent advances in biotechnology and particularly owing to the advent of nanotechnology, separation, and identification of new chemical entities at minute concentrations are possible, which may open new avenues for herbal drugs [635].

There is an urgent need to assess the potential usage of medicinal plants and health care products derived from their origin, in modern medicinal aspect, to enhance the current market potential [12, 19, $56,67,614,636]$. Plant extract contains a plethora of compounds and each compound in the extract may not be safe, biologically active and efficient. Thus, Food and Drug Administration (FDA) has to check the safety of each compound in the extract. However, nanotechnology-based 'smart' drug delivery systems and nanocarriers can be used for efficient delivery of the phytochemical-based drugs to the target organs, that will enhance their judicious use. Nano-carriers possess a high potential to pass physiological barriers like a blood-brain barrier and tight epithelial junctions of the skin. Also, bioavailability, control, facile and efficient release time and precise drug targeting, etc. are among other requisite characteristics [632]. Likewise, the use of nano-suspensions, nano-sensors, and nanotubes for the efficient delivery of the various phytomedicinal preparations is quite noteworthy in cancer treatment.

On the other hand, for medicinal purpose, toxicological evaluation, regulatory guidelines, quality control and dose standardization are among mandatory criterion for a successful implementation and launching of drugs/natural products. In this regard, comprehensive databases likewise, Napralert, Pharmel, Napreca and Traditional Knowledge Digital Library (TKDL) along with high-tech analytical methods and good clinical practice (GCP) performances are needed for a precise information and dissemination of knowledge on medicinal plants. The other main challenges in the field of Phytomedicine include but not limited to the lack of scientific documentation and records available that have resulted in many species are becoming extinct. This is also because of the lack of collaboration between phytomedicinal, traditional medical and orthodox medical practitioners, and scientists led to no 
sharing of scientific knowledge, lack of a bench to bedside technology transfer and clinical trials needed for validation. Moreover, the majority of the naturally occurring plant-based herbal products lack scientific evidence, which hampers its clinical use. Furthermore, incorrect/inappropriate manufacturing practices such as misidentification, lack of standardization, low-quality manufacturing tools, microbial contamination, poor or improper packaging, inappropriate maintenance/storage (temperature, light exposure, etc), substitution and adulteration of plants, incorrect preparation and dosage are some constraints for regular use of phytomedicines, thus should be taken with an utmost care.

During the past several years, researchers are redirecting their interests to revalorize the natural herbs and their medicinal value as compared to the synthetic counterparts and alternatives. Moreover, words like microbial resistance, drug failure, and significant side-effects are emphasized in growing medicinal awareness. Therefore, people are more awakened to go for safer and natural alternatives like herbs and plant-derived metabolites. Also, recent advancements in the biotechnological and biomedical world at the molecular level have also allowed the elucidation of mechanism based receptor screens to probe interactions between large molecules. Moreover, with the advent of technology at nano- level, naturally occurring small product molecules are being considered as potential drug candidates for therapy and prophylaxis, as a new era of treatment. The utilization of nanotubes, nanowires, nano-sensors, and nano-suspensions could have considerable interests for an efficient and switchable delivery of various phytomedicinal preparations for cancer treatment and also tackle other diseases involving central nervous system.

In conclusion, a considerable scope exists to implement the above discussed novel technologies at various levels, i.e., Macro, Micro, and Nano in the field of medicine and veterinary sciences. Given the long-term striving for socially acceptable, sustainable and friendly biotechnological processes, the health concerns regarding harmful chemicals, the versatility, fewer side-effect, and high-level bioactivity, herbal heritage with an aim to develop new phytomedicines is likely to remain the subject of intensive research investigations in different sectors of the modern world. Moreover, this could also help in revolutionizing and widen the use and applicability of the naturally occurring rich and novel wealth for numerous health benefits for humans and animals alike.

\section{Conclusion and future perspectives}

The importance of medicinal plants as a complementary and alternative remedy is growing the pharmaceutical industry development along with the advances in research and development in the area of drug discovery. The increasing number and quality of novel and more efficient biological, synthetic and semisynthetic pharmacological products have not impacted the importance of natural products in medicine, especially in the developing world. In contrast, the fast growing population of the world particularly those in the developing countries has increased the demand for medicinal plants themselves and the products derived from them. This is because herbal medicines including herbal immunomodulators represent many unrealized promises. It necessitated the wealth of traditional knowledge and continued the search for the unexplored therapeutic potential of other plants. A wide range of herbal plants contains different groups of phytochemicals like triterpene saponins, alkaloids, flavonoids, pectins, diterpenoids, lignins, etc. These bioactive constituents, along with many other mediators and mutual interactions, are well known to possess cytoprotective, immunomodulatory, antioxidant, antitumor, anti-inflammatory, anticancer and antibacterial activities through different mechanisms viz., some of them help in activation and promoting the production of many types of proinflammatory cytokines like IL-1, IL-2, IL-6, IL-8, IL-10, IL-12 and some interferons like IFN- $\gamma$ and TNF- $\alpha$ from macrophage beside enhancing the cytotoxic effects of macrophages. The active biological components extracted from various plants suggested to be with immunological effects have been proven to exhibit immune stimulation through increasing antibody production, phagocytosis, phagocytic index, activation of natural killer (NK) cells, complement system and $\mathrm{Th}_{1}$-pathway cytokines and elevation of both neutrophil and lymphocyte count. Many extracts can act through modulation of lymphocyte proliferation activity and lymphocyte-mediated immune functions especially CD4 (+) T cells and $\mathrm{B}$ and increase the population of CD3, CD4, CD8 cells while other extracts have been reported to have beneficial impacts on albumin and different types of immunoglobulins.

The ability of phytochemicals extracted from medicinal plants to inhibit the cyclooxygenases and 5-lipoxygenase pathways and their effective antibacterial activity give them the improved 
pharmacological properties and therapeutic potential. In future, detailed chemical and pharmacological investigations of the traditional formulations and medicinal plants will be very helpful for inventing/developing the new veterinary drug. Some limitations regarding the large-scale exploration and therapeutical use of plant-based drugs and associated preparations exist presently which include the inadequate amount of phytochemicals after extraction, low bioavailability of the constituents, lack of standardized quality control systems, etc. So these issues needed to be addressed by exploring novel isolation techniques, efficient and targeted drug delivery systems, developing standardized quality control strategies, etc. for the fruitful and affluent implementation/ application of immune modulating for various ailments. The way out may involve proposals including conservation of plant biodiversity by creating botanical gardens of medicinal herbs, increased training courses in herbal veterinary medicine to appreciate and refine the conventional wisdom. Added efforts are required to produce evidence for the value of herbal medicines with randomized and controlled clinical trials. Moreover, evaluation of traditional remedies for their efficacy and safety may become a basis for their future classification, and it will serve to identify the potential use of herbal medicines and their processed products in national health care system throughout the world.

\section{Conflicts of interest}

All authors declare that there exist no commercial or financial relationships that could in any way lead to a potential conflict of interest.

\section{Acknowledgements}

All the authors acknowledge and thank their respective Institutes and Universities for providing literature facilities.

\section{References}

[1]. $\quad$ Nestler, G. Traditional Chinese medicine. Med. Clin. N. Am., 2002, 86, 1-9.

[2]. Archana, Jatawa, S.; Paul, R.; Tiwari, A. Indian Medicinal Plants: A Rich Source of Natural Immuno-Modulator. Inter. J. Pharmacol., 2011, 7, 198-205.

[3]. Konkimalla, V.B.; Efferth, T. Evidence-based Chinese medicine for cancer therapy. J. Ethnopharmacol., 2008, 116(2), 207-210.

[4]. Olaku, O.; White, J.D. Herbal therapy use by cancer patients: A literature review on case reports. Europ. J. Cancer., 2011, 47(4), 508-514.

[5]. Dhama, K.; Mani, S.; Chakraborty, S.; Tiwari, R.; Kumar, A.; Selvaraj, P.; Rai, R.B. Herbal remedies to combat cancers in humans and animals - a review. Int. J. Curr. Res., 2013, 5(07), 1908-1919.

[6]. He, D.Y.; Dai, S.M. Anti-inflammatory and immunomodulatory effects of Paeonia lactiflora Pall., a traditional Chinese herbal medicine. Front. Pharmacol., 2011, 2(10), 1-5.

[7]. Yang, Y.; Zhang, Z.; LI, S.; Xiaoli, Y.; Li, X.; He, K. Synergy effects of herb extracts: pharmacokinetics and pharmacodynamic basis. Fitoterapia, 2014, 92, 133-147.

[8]. Ngeh, J.T.; Jacob, W.; Nuwanyakpa, Sali, D. Ethnoveterinary medicine. Agromisa Foundation and CTA, Wageningen., 2007.

[9]. Arreola, R.; Quintero-Fabián, S.; López-Roa, R.I.; Flores-Gutiérrez, E.O.; Reyes-Grajeda, J.P.; Carrera-Quintanar, L.; Ortuño-Sahagún, D. Immunomodulation and anti-inflammatory effects of garlic compounds. J. Immunol. Res., 2015, e401630. doi: 10.1155/2015/401630.

[10]. Zhang, L.; Koyyalamudi, S.R.; Jeong, S.C.; Reddy, N.; Smith, P.T.; Ananthan, R.; Longvah, T. Antioxidant and immunomodulatory activities of polysaccharides from the roots of Sanguisorba officinalis. Int. J. Biol. Macromol., 2012, 51(5), 1057-1062.

[11]. Patil, U.S.; Jaydeokar, A.V. Bandawane, D.D. Immunomodulators: A pharmacological review. Int. J. Pharm. Pharm. Sci., 2012, 4, 30-36.

[12]. Das, S.; Bordoloi, R.; Newar, N. A Review on Immune Modulatory Effect of Some Traditional Medicinal Herbs. $J$. Pharmaceut. Chem. Biol. Sci., 2014, 2(1), 33-42.

[13]. Dhama, K.; Kesavan, M.; Karthik, K.; Amarpal, Tiwari, R.; Sunkara, L.T.; Singh, R.K. Neuroimmunomodulation countering various diseases, disorders, infections, stress and aging. Int. J. Pharmacol., 2015a, 11(2), 76-94.

[14]. Thatte, U.M.; Dahanukar, S.A. Rasayana concept: clues from immunomodulatory therapy. In: Upadhyay SN (ed.) Immunomodulation. Narosa Publishing House, New Delhi, 1997, 141.

[15]. Chulet, R, P. Pradhan .A review on rasayana. Phcog. Rev., 2009, 3, 229-234.

[16]. Sohni, Y.R.; Kaimal, P.; Bhatt, R.M. The antiamoebic effect of a crude drug formulation of herbal extracts against Entamoeba histolytica in vitro and invivo. J. Ethanopharmacol., 1995, 45, 43-52. 
[17]. Dhuley, J.N. Effect of some Indian herbs on macrophage function in ochratoxin A treated mice. Ethnopharmacol., 1997, 58, 15-20.

[18]. Upadhyay, S.N. Proceedings of the International Ayurveda Conference- 97. Sanjay Gandhi Post Graduate Institute of Medical Sciences, Lucknow. 1997, 10.

[19]. Mahima, Rahal, A.; Deb, R.; Latheef, S.K.; Samad, H.A.; Tiwari, R.; Verma, A.K.; Kumar, A. Dhama, K. Immunomodulatory and therapeutic potentials of herbal, traditional / indigenous and ethnoveterinary medicines. Pak. J. Biol. Sci., 2012, 15(16), 754-774.

[20]. Gholamnezhada, Z.; Keyhanmanesh, R.; Boskabady, M.H. Anti-inflammatory, antioxidant and immunomodulatory aspects of Nigella sativa for its preventive and bronchodilatory effects on obstructive respiratory diseases: A review of basic and clinical evidence. J. Funct. Foods, 2015, 17, 910-927.

[21]. Alagawany, M.; Abd El-Hack, M.E.; El-Kholy, M.S. Productive performance, egg quality, blood constituents, immune functions, and antioxidant parameters in laying hens fed diets with different levels of Yucca Schidigera extract. Env. Sci. Pollution Res., 2016a, 23, 6774-6782.

[22]. Alagawany, M.; Ashour, E.A.; Reda, F.M. Effect of dietary supplementation of garlic (Allium sativum) and turmeric (Curcuma longa) on growth performance, carcass traits, blood profile and oxidative status in growing rabbits. Ann. Anim. Sci., 2016b, 16(2), 1-17.

[23]. Rahal, A.; Mahima, Verma, A.K.; Kumar, A.; Tiwari, R.; Kapoor, S.; Chakraborty, S. and Dhama, K. Phytonutrients and nutraceuticals in vegetables and their multi-dimensional medicinal and health benefits for humans and their companion animals: A review. J. Biol. Sci., 2014, 14, 1-19.

[24]. Bhushan, P.; Manish, G. Botanical immunodrugs: Scopes and opportunities. Drug Dis. Today, 2005, 10(7), 495-502.

[25]. Niemeyer, K,; Bell, I.R.; Koithan, M. Traditional knowledge of western herbal medicine and complex systems science. J. Herb Med., 2013, 3(3), 112-119.

[26]. Farnsworth, N.R. The role of ethnopharmacology in drug development, Bioactive compounds from plants. Ciba Foundation Symposium Wile, 1990, 154, 2-11.

[27]. Bala, M.; Pratap, K.; Verma, P.K.; Singh, B.; Padwad, Y. Validation of ethnomedicinal potential of Tinospora cordifolia for anticancer and immunomodulatory activities and quantification of bioactive molecules by HPTLC. $J$. Ethnopharmacol., 2015, 175,131-137.

[28]. Dai, Y.; Kato, M.; Takeda, K.; Kawamoto, Y.; Akhand, A.A.; Hossain, K.; Suzuki, H.; Nakashima, I. T-cellimmunity-based inhibitory effects of orally administered herbal medicine juzen-taiho-to on the growth of primarily developed melanocytic tumors in RET-transgenic mice. J. Investig. Dermatol., 2001, 117, 694-701.

[29]. Plaeger, S.F. Clinical immunology and traditional herbal medicines. Clin. Diagn Lab Immunol., 2003, 10, $337-338$.

[30]. Umashanker, M.; Shruti, S. Traditional Indian herbal medicine used as antipyretic, antiulcer, anti-diabetic and anticancer: A review. Int. J. Res. Pharm. Chem., 2011, 1(4), 1152-1159.

[31]. Dhama, K.; Tiwari, R.; Chakraborty, S.; Saminathan, M.; Kumar, A.; Karthik, K.; Wani, M.Y.; Amarpal; Singh, S.V.; Rahal, A. Evidence based antibacterial potentials of medicinal plants and herbs countering bacterial pathogens especially in the era of emerging drug resistance: An integrated update. Int. J. Pharmacology, 2014a, 10(1), 1-43.

[32]. Rahal, A.; Kumar, A.; Singh, V.; Yadav, B.; Tiwari, R.; Chakraborty, S.; Dhama, K. Oxidative stress, prooxidants and antioxidants: the interplay. BioMed Res. Int. 2014, e761264, http://dx.doi.org/10.1155/2014/761264.

[33]. Dar, N.J.; Hamid, A.; Ahmad, M. Pharmacological overview of Withania somnifera, the Indian Ginseng. Cell Mol. Life Sci., 2015, 72(23), 4445-4460.

[34]. Singh, S.K.; Rajoria, K. Ayurvedic management in cervical spondylotic myelopathy. J. Ayurveda Integr. Med., 2017, S0975-9476(16)30195-4.

[35]. Tan, B.K.; Vanitha, J. Immunomodulatory and antimicrobial effects of some traditional Chinese medicinal herbs: a review. Curr. Med. Chem., 2004, 11, 1423-1430.

[36]. Abonyi, D.O.; Adikwu, M.U.; Esimone, C.O.; Ibezim, E.C.; Plants as source of antiviral agents. Afr. J. Biotechnol., 2009, 8(17), 3989-3994.

[37]. Marathe, S.A.; Datey, A.A.; Chakravortty, D. Herbal cocktail as anti-infective: promising therapeutic for the treatment of viral diseases. Recent Pat. Antiinfect. Drug Disc., 2012, 7(2), 123-132.

[38]. Huang, J.; Su, D.; Feng, Y.; Liu, K.; Song, Y. Antiviral herbs-Present and future. Infect. Disord. Drug Targets, 2014, $14,61-73$.

[39]. Ganjhu, R.K.; Mudgal, PP.; Maity, H.; Dowarha, D.; Devadiga, S.; Nag, S.; Arunkumar, G. Herbal plants and plant preparations as remedial approach for viral diseases. Virus Dis., 2015, 26(4), 225-36.

[40]. Singh, M.K.; Yadav, S.S.; Yadav, R.S.; Chauhan, A.; Katiyar, D.; Khattri, S. Protective effect of Emblica-officinalis in arsenic induced biochemical alteration and inflammation in mice. Springerplus, 2015, 4, 438.

[41]. Liu, Q.; Zhou, Y.H.; Yang, Z.Q. The cytokine storm of severe influenza and development of immunomodulatory therapy. Cell. Mol. Immunol., 2016, 13(1), 3-10.

[42]. Williamson EM. 2001. Synergy and other interactions in phytomedicines. Phytomedicine 8: 401-409.

[43]. Kerr, C. Curry ingredient protects skin against radiation. The Lancet Oncology, 2002, 3, 713.

[44]. Spelman, K.; Burns, J.; Nichols, D.; Winters, N.; Ottersberg, S.; Tenborg, M. Modulation of cytokine expression by traditional medicines: A review of herbal immunomodulators. Altern. Med. Rev., 2006, 11(2), 128-150.

[45]. Baliga, M.S.; Meera, S.; Mathai, B.; Rai, M.P.; Pawar, V.; Palatty, P.L.; Scientific validation of the ethnomedicinal properties of the Ayurvedic drug Triphala: A review. Chin. J. Integr. Med., 2012, 18, 946-954.

[46]. Csermely, P.; Agoston, V.; Pongor, S. The efficiency of multi-target drugs: the network approach might help drug design. Trends Pharmacol. Sci., 2005, 26, 178-182.

[47]. Keith CT, Borisy AA, Stockwell BR. Multicomponent therapeutics for networked systems. Nat. Rev. Drug Discov., 2005, 4, 71-78. 
[48]. Choi, H.R.; Nam, K.; Lee, H.; Yang, S.; Kim, Y.; Lee, J.; Date, A.; Toyama, K.; Park, K. Phlorizin, an active ingredient of eleutherococcus senticosus, increases proliferative potential of keratinocytes with inhibition of mir135b and increased expression of type IV collagen. Oxid. Med. Cell. Long., 2016, e3859721.

[49]. Aghsaghali, A.M. Importance of medical herbs in animal feeding: A review. Ann. Biol. Res., 2012, 3(2), 918-923.

[50]. Dhama, K.; Tiwari, R.; Khan, R.U.; Chakraborty, S.; Gopi, M,; Karthik, K.;Saminathan, M.; Desingu, P.A.; Sunkara, L.T. Growth promoters and novel feed additives improving poultry production and health, bioactive principles and beneficial applications: The trends and advances- A Review. Int. J. Pharmacology, 2014b, 10(3), 129-159.

[51]. Dhama, K.; Latheef, S.K.; Mani, S.; Abdul Samad H.; Karthik, K.; Tiwari, R.; Khan, R.U.; Alagawany, M.; Mayada, R.F.; Alam, G.M.; Laudadio, V.; Tufarelli, V. Multiple beneficial applications and modes of action of herbs in poultry health and production. A Review. Int. J. Pharmacol, 2015b, 11, 152-176.

[52]. Alagawany, M.; Abd El-Hack, M.E.; Farag, M.R.; Tiwari, R.; Dhama, K. Biological effects and modes of action of carvacrol in animal and poultry production and health: A review. Adv. Anim. Vet. Sci., 2015a, 3, 73-84.

[53]. Alagawany, M.M.; Farag, M.R.; Dhama, K.; Abd El-Hack, M.E.; Tiwari, R.; Alam, G.M. Mechanisms and beneficial applications of resveratrol as feed additive in animal and poultry nutrition. A review. Int. J. Pharmacol., 2015b, 11(3), 213-221.

[54]. Xie, P.; Chen, S.; Liang, Y.; Wang, X.; Tian, R.; Upton, R. Chromatographic fingerprint analysis--a rational approach for quality assessment of traditional Chinese herbal medicine. J. Chromatogra A., 2006, 1112, 171-180.

[55]. Mosihuzzaman, M. Herbal medicine in healthcare-an overview. Nat. Prod. Commun., 2012, 7(6), 807-812.

[56]. Verma, S. and Singh, S.P. Current and future status of herbal medicines. Vet. World., 2008, 1(11), 347-350.

[57]. Citarasu, T. Herbal biomedicines: a new opportunity for aquaculture industry. Aqua. Int., 2010, 18, 403-414.

[58]. Mahima, Ingle, A.M.; Verma, A.K.; Tiwari, R.; Karthik, K.; Chakraborty, S.; Deb, R.; Rajagunalan, S.; Rathore, R.; Dhama, K. Immunomodulators in day to day life: a review. Pak. J. Biol. Sci., 2013, 16(17), 826-843.

[59]. Zahran, E.; Risha, E.; Abdel-hamed, F.; Mahgoub, H.A.; Ibrahim, T. Effects of dietary Astragalus polysaccharides (APS) on growth performance, immunological parameters, digestive enzymes, and intestinal morphology of Nile tilapia (Oreochromis niloticus). Fish Shellfish Immunol., 2014, 38(1), 149-157.

[60]. Anjaria, J.; Parabia, M.; Dwivedi, S. Ethnovet Heritage: Indian ethnoveterinary medicine: an overview. Pathik publishers, Ahmedabad, India, 2002.

[61]. Somvanshi, R. Veterinary Medicine and Animal Keeping in Ancient India. Asian Agri-Hist., 2006, 10(2), $133-146$.

[62]. Puri, H.S. 'Rasayana'-Ayurvedic herbs for longevity and rejuvenation. Taylor and Francis, London. 2003.

[63]. Rege, N.N.; Thatte, U.M.; Dahanukar, S.A. Adaptogenic properties of six Rasayana herbs used in Ayurvedic medicine. Phytother. Res., 1999, 13, 275-291.

[64]. Brahma, S.K.; Debnath, P.K. Therapeutic importance of Rasayana drugs with special reference to their multidimensional actions. Aryavaidyan, 2003, 16, 160-163.

[65]. Balasubramani, S.P.; Venkatasubramanian, P.; Kukkupuni, S.K.; Patwardhan, B. Plant-based Rasayana drugs from Ayurveda. Chin. J. Integr. Med., 2011, 17(2), 88-94.

[66]. Wagner, H. Therapy and prevention with immunomodulatory and adaptogenic plant drugs. Update Ayurveda, 1994, 24-26.

[67]. Paramkusha, R.M. Ayurveda rasayana drugs: A review on current research. World J. Pharm. Res., 2015, 4(11), 843858.

[68]. Agarwal, S.S.; Singh, V.K. Immunomodulators: a review of studies on Indian medicinal plants and synthetic peptides. Proc. Indian Sci. Acad., 1999, 65B, 179-204.

[69]. Scartezzini, P.; Speroni, E. Review on some plants of Indian traditionalmedicine with antioxidant activity. $J$ Ethnopharmacol., 2000, 71, 23-43.

[70]. Gharagozloo, M.; Ghaderi, A. Immunomodulatory effect of concentrated lime juice extract on activated human mononuclear cells. J. Ethnopharmacol., 2001, 77(1), 85-90.

[71]. Kim, Y.H.; Ha, J.C.; Do, J.S.; Choi, Y.H.; Choo, Y.K.; Woo, W.H.; Yi, H.K.; Hwang, P.H.; Nam, S.Y. In vitro immunomodulatory activity of Bo-yang-hwan-o-tang. Immunopharmacol. Immunotoxicol., 2004, 26(4), 631-644.

[72]. Kaileh, M.; Berghe, W.V.; Boone, E.; Essawi, T.; Haegeman, G. Screening of indigenous Palestinian medicinal plants for potential anti-inflammatory and cytotoxic activity. J. Ethnopharmacol., 2007, 13(3), 510-516.

[73]. Amirghofran, Z.; Bahmani, M.; Azadmehr, A.; Javidnia, K.; Miri, R. Immunomodulatory activities of various medicinal plant extracts: effects on human lymphocytes apoptosis. Immunol. Invest., 2009, 38(2), 181-189.

[74]. Anton, M.; Barbara, M. A new concept in prophylaxis and therapy: paramunization by poxvirus inducers. Pesq. Vet. Bras., 1999, 19(3/4), 91-98.

[75]. Dhama, K.; Latheef, S.K.; Mani, S.; Samad, H..; Karthik, A.K.; Tiwari, R.; Khan, R.U. Multiple beneficial applications and modes of action of herbs in poultry health and production-A review. Inter. J. Pharmacol., 2015, 11 (3), 152-176.

[76]. Spickler, A.R.; Roth, J.A. Adjuvants in veterinary vaccines: modes of action and adverse effects. J. Vet. Intern. Med. 2003, 17(3), 273-281.

[77]. Rajput, Z.I.; Hu, S.H.; Xiao, C.W.; Arijo, A.G. Adjuvant effects of saponins on animal immune responses. J. Zhejiang Univ. Sci. B., 2007, 8(3), 153-161.

[78]. Sun, H.X.; Xie, Y.; Ye, Y.P. ISCOMs and ISCOMATRIX. Vaccine, 2009, 27(33), 4388-4401.

[79]. Kumazawa, Y.; Itagaki, A.; Fukumoto, M.; Fujisawa, H.; Nishimura, C.; Nomoto, K. Activation of peritoneal macrophages by berberinetype alkaloids in terms of induction of cytostatic activity. Int. J. Immunopharmacol., 1984, $6,587-592$. 
[80]. Roesler, J.; Emmendorffer, A.; Steinmuller, C.; Luettig, B.; Wagner, H.; Lohmann-Matthes, M.L. Application of purified polysaccharides from cell cultures of the plant Echinacea purpurea to test subjects mediates activation of the phagocyte system. Int. J. Immunopharmacol., 1991, 13, 931-941.

[81]. Puri, A.; Saxena, R.; Saxena, R.P.; Saxena, K.C.; Srivastava, V.; Tandon, J.S. Immunostimulant agents from Andrographis paniculata. J. Nat. Prod., 1993, 56, 995-999.

[82]. Vasanthakumar, P.; Pangayarselvi, B.; Sasikumar, P.; Chandrasekaran, D.; Doraisamy, K.A.; Purushothaman, M.R. Performance of broilers fed ashwagandha (Withania somnifera) incorporated diets during summer season for alleviating heat stress. Indian J. Anim. Res., 2015, 49(3), 333-335.

[83]. Singh, R.K. Tinospora cordifolia as an adjuvant drug in the treatment of hyper-reactive malarious splenomegaly - case reports. J. Vect. Borne. Dis., 2005, 3, 36-38.

[84]. $\quad$ Ragupathi, G.; Yeung, K.S.; Leung, P.C.; Leee, M.; Lau, C.B.; Vickers, A.; Hood, C.; Deng, G,; Cheung, N.K.; Cassileth, B.; Livingston, P. Evaluation of widely consumed botanicals as immunological adjuvants. Vaccine, 2008, $26,4860-4865$.

[85]. Tan, L.; Wei, T.; Yuan, A.; He, J.; Liu, J.; Xu, D.; Yang, Q. Dietary supplementation of Astragalus polysaccharides enhanced immune components and growth factors egf and igf-1 in sow colostrum. J. Immunol. Res., 2017, e 9253208, 9253208. http://doi.org/10.1155/2017/9253208.

[86]. Saravanan, S.; Pandikumar, P.; Prakash Babu, N.; Hairul Islam, VI.; Thirugnanasambantham, K, Gabriel Paulraj, M.; Balakrishna, K.; Ignacimuthu, S. In vivo and in vitro immunomodulatory potential of swertiamarin isolated from Enicostema axillare (Lam.) A. Raynal that acts as an anti-inflammatory agent. Inflammation., 2014, 37(5), 1374-1388.

[87]. Bhajoni, P.S.; Meshram, G.G.; Lahkar, M.; Ghadlinge, M.S.; Rehan, H.S.; Tripath, C.D. Evaluation of the role of nitric oxide and antioxidation in the antiulcer activity of Ocimum sanctum leaves: an experimental study. Orient. Pharma. Exp. Med., 2015, 15(4), 313-317.

[88]. Kan, X.; Zhang, W.; You, R.; Niu, Y.; Guo, J.; Xue, J. Scutellaria barbata D. Don extract inhibits the tumor growth through down-regulating of Treg cells and manipulating Th1/Th17 immune response in hepatoma H22-bearing mice. BMC Complement. Alter. Med., 2017, 17, 41. http://doi.org/10.1186/s12906-016-1551-9.

[89]. Gupta, V.K.; Fatima, A.; Faridi, U.; Negi, A.S.; Shanker, K.; Kumar, J.K.; Rahuja, N.; Luqman, S.; Sisodia, B.S.; Saikia, D.; Darokar, M.P.; Khanuja, S.P. Antimicrobial potential of Glycyrrhiza glabra roots. J. Ethnopharmacol. 2008, 116(2), 377-380.

[90]. Obolentseva, G.V.; Litvinenko, V.I.; Ammosov, A.S. Pharmacological and therapeutic properties of licorice preparations (a review). Pharm. Chem. J., 1999, 33, 24-31.

[91]. Yamamura, Y.; Kawakami, J.; Santa, T. Pharmacokinetic profile of glycyrrhizin in healthy volunteers by a new highperformance liquid chromatographic method. J. Pharm. Sci., 1992, 8, 1042-1046.

[92]. Ohuchi, K.; Tsurufuji, A. A study of the anti-inflammatory mechanism of glycyrrhizin. Mino. Med. Rev., 1982, 27, 188-193.

[93]. Sekizawa, T.; Yanagi, K.; Itoyama, Y. Glycyrrhizin increases survival of mice with herpes simplex encephalitis. Acta Virol., 2001, 45, 51-54.

[94]. Nagai, T.; Egashira, T.; Yamanaka, Y.; Kohno, M. The protective effect of glycyrrhizin against injury of the liver caused by ischemia-reperfusion. Arch. Env. Contam. Toxicol., 1991, 20, 432-436.

[95]. Yoshikawa, M.; Matsui, Y.; Kawamoto, H.; Umemoto, N.; Oku, K.; Koizumi, M.; Yamao, J.; Kuriyama, S.; Nakano H.; Hozumi, N.; Ishizaka, S.; Fukui, H. Effects of glycyrrhizin on immune-mediated cytotoxicity. J. Gastroenterol. Hepatol., 1997, 12, 243-248.

[96]. Guo, A.; He, D.; Xu, H.; Geng, C.; Zhao, J. Promotion of regulatory T cell induction by immunomodulatory herbal medicine licorice and its two constituents. Scient. Rep., 2016, 5, 14046.

[97]. Dorhoi, A.; Dobrean, V.; Zăhan, M.; Virag, P. Modulatory effects of several herbal extracts on avian peripheral blood cell immune responses. Phytother. Res., 2006, 20(5), 352-358.

[98]. Berezin, V.E.; Bogoyavlenskyi, A.P.; Khudiakova, S.S.; Alexuk, P.G.; Omirtaeva, E.S.; Zaitceva, I.A.; Tustikbaeva, G.B.; Barfield, R.C.; Fetterer, R.H. Immunostimulatory complexes containing Eimeria tenella antigens and low toxicity plant saponins induce antibody response and provide protection from challenge in broiler chickens. Vet. Parasitol., 2010, 67(1), 28-35.

[99]. Zhang, Q.; Ye, M. Chemical analysis of the Chinese herbal medicine Gan-Cao (licorice). J. Chromatogr., 2009, 13, 1216(11), 1954-1969.

[100]. Ayeka, P.A.; Bian, Y.; Mwitari, P.G.; Chu, X.; Zhang, Y.; Uzayisenga, R.; Otachi, E.O. Immunomodulatory and anticancer potential of Gan cao (Glycyrrhiza uralensis Fisch.) polysaccharides by CT-26 colon carcinoma cell growth inhibition and cytokine IL-7 upregulation in vitro. BMC Complement. Altern. Med., 2016, 16, 206.

[101]. Jessica Houdret. Glycyrrhiza glabra In: Practical Herb Garden Anness publishing Ltd. Hermes House 88-89 Blackfriars Road, London SEI 8HA, 2003c, 160.

[102]. Dinesh, N.; Neelagiri, S.; Kumar, V.; Singh, S. Glycyrrhizic acid attenuates growth of Leishmania donovani by depleting ergosterol levels. Exp. Parasitol., 2017, 176, 21-29.

[103]. Hosseinzadeh, H.; Nassiri-Asl, M. Pharmacological effects of Glycyrrhiza spp. and its bioactive constituents: Update and review. Phytother. Res., 2015, 29(12), 1868-1886.

[104]. Shams, K.A.; Abdel-Azim, N.S.; Kamal, S.A.; Husseiny, H.A.; Hammouda, F.M. Green extraction: Enhanced extraction yield of glycyrrhizic acid from Glycyrrhiza glabra L. J. Chem. Pharm. Res., 2015, 7(4), 725-728.

[105]. Shuai, J.; Ziwei, L.; Wei, S.; Yongrui, W.; Wenfei, L.; Kai, L.; Shunan, T.; Qi, W.; Xue, Q.; Demin, Z.; Siwang, Y.; Min, Y. Bioactive constituents of glycyrrhiza uralensis (licorice): discovery of the effective components of a traditional herbal medicine. J. Nat. Prod., 2016, 79(2), 281-292. 
[106]. Asha, R.; Navneet, K.V.; Chaudhari, S.K.; Vikash, C.; Devendra, P.S.; Manjo, K.P. Phytochemical constituent, Pharmacological activities and Medicinal uses through the millennia of Glycyrrhiza glabra LINN: A Review. Inter. J. Pharm., 2012, 3(8), 45-55.

[107]. Castleman, M. Ashwagandha; Echinacea; Eleuthero; Turmeric (Curcuma longa) In: The new healing herbs Rondale Inc USA. 2009a, 190-196.

[108]. Mishra, L.C.; Singh, B.B.; Dagenais S. Scientific basis for the therapeutic use of Withania somnifera (Ashwagandha): a review. Altern. Med. Rev., 2000, 5.

[109]. Tiwari, R.; Chakraborty, S.; Saminathan, M.; Dhama, K.; Singh, S.V. Ashwagandha (Withania somnifera): Role in safeguarding health, immunomodulatory effects, combating infections and therapeutic applications: A review. J. Biol. Sci., 2014a, 14(2), 77-94.

[110]. Patil, D.; Gautam, M.; Mishra, S.; Karupothula, S.; Gairola, S.; Jadhav, S.; Pawar, S.; Patwardhan, B. Determination of withaferin $\mathrm{A}$ and Withanolide $\mathrm{A}$ in mice plasma using high performance liquid chromatography-tandem mass spectrometry: Application to pharmacokinetics after oral administration of Withania somnifera aqueous extract. $J$. Pharmaceutical Biomed. Analysis, 2013, 80, 203-212.

[111]. Thaiparambil, J.T.; Bender, L.; Ganesh, T.; Kline, E.; Patel, P. Withaferin A inhibits breast cancer invasion and metastasis at sub-cytotoxic doses by inducing vimentin disassembly and serine 56 phosphorylation. Int. J. Cancer, 2011, 129, 2744-2755.

[112]. Dahikar, P.R.; Kumar, N.; Sahni, Y.P. Pharmacokinetics of Withania somnifera (Ashwagandha) in healthy buffalo calves. Buffalo Bull., 2012, 31(4), 219-223.

[113]. Sumanth, M.; Nedunuri, S. Comparison of bioavailability and bioequivalence of herbal anxiolytic drugs with marketed drug alprazolam. World J. Pharm. Res., 2014, 3, 1358-1366.

[114]. Abdallah, N.M.; Noaman, E.; Eltahawy, N.A.; Badawi, A.M.; Kandil, E.; Mansour, N.A.; Mohamed, H.E. Anticancer and radiosensitization efficacy of nanocomposite withania somnifera extract in mice bearing tumor cells. Asian Pac. J. Cancer Prev., 2016, 17(9), 4367-4375.

[115]. White, P.T.; Subramanian, C.; Motiwala, H.F.; Cohen, M.S. Natural Withanolides in the treatment of Chronic Diseases. Adv. Exp. Med. Biol., 2016, 2928, 329-373.

[116]. Henley AB, Yang L, Chuang KL, Sahuri-Arisoylu M, Wu LH, Bligh SW, Bell JD. Withania somnifera Root Extract Enhances Chemotherapy through 'Priming'. PLoS One, 2017, 12(1), e0170917.

[117]. Iuvone, T.; Esposito, G.; Capasso, F.; Izzo, A.A. Induction of nitric oxide synthase expression by Withania somnifera in macrophages. Life Sci., 2003, 72, 1617-1625.

[118]. Jayaprakasam, B.; Zhang, Y.; Seeram, N.P.; Nair, M.G. Growth inhibition of human tumor cell lines by withanolides from Withania somnifera leaves. Life Sci., 2003, 74, 125-132.

[119]. Davis, L.; Kuttan, G. Effect of Withania somnifera on cell mediated immune responses in mice. J. Exp. Clin. Cancer Res., 2002, 21, 585-590.

[120]. Winters, M. Ancient medicine, modern use: Withania somnifera and its potential role in integrative oncology. Altern. Med. Rev., 2006, 11(4), 269-277.

[121]. Ziauddin, M.; Phansalkar, N.; Patki, P.; Diwanay, S.; Patwardhan, B. Studies on the immunomodulatory effects of Ashwagandha. J. Ethnopharmacol., 1996, 50(2), 69-76.

[122]. Kumaria, P.; Singh, S.K.; Dimri, U.; Kataria, M.; Ahlawat, S. Immunostimulatory activities of Withania somnifera root extract in dexamethasone induced immunocompromised mice and in vitro model. Asian J. Complement. Alter. Med., 2014, 02(02), 06-10.

[123]. Ali, M.; Rizvi, T.F.; Alam, S.P.; Ali, M.C.; Kumar, R.; Kumar, A. Immunomodulatory effect of Withania somnifera (Ashwagandha) on cyclophosphamide induced toxicity in rats. Am. J. PharmTech. Res., 2015, 5(3), 638-645.

[124]. Malik, F.; Singh, J.; Khajuria, A.; Suri, K.A.; Satti, N.K.; Singh, S.; Kaul, M.K.; Kumar, A.; Bhatia, A.; Qazi, G.N. A standardized root extract of Withania somnifera and its major constituent withanolide-A elicit humoral and cellmediated immune responses by up regulation of Th1-dominant polarization in BALB/c mice. Life Sci., 2007, 80(16), 1525-1538.

[125]. El-Boshy, Mel-S.; Abdalla, O.M.; Risha, A.; Moustafa, F. Effect of Withania somnifera extracts on some selective biochemical, hematological, and immunological parameters in guinea pigs experimental infected with E. coli. ISRN Vet. Sci., 2013, e153427.

[126]. Kaur, T.; Singh, H.; Mishra, R.; Manchanda, S.; Gupta, M.; Saini, V.; Sharma, A.; Kaur, G. Withania somnifera as a potential anxiolytic and immunomodulatory agent in acute sleep deprived female Wistar rats. Mol. Cell. Biochem., 2017, 427(1-2), 91-101.

[127]. Chauhan, R.S. Effect of immuplus on humoral and cell mediated immunity in dogs. J. Immunol. Immunopathol., 1999, 1, 54-57.

[128]. Dhote, B.S.; Singh, G.K.; Chauhan, R.S. Effect of immuplus (a herbal immunomodulator) on paraspecific immune responses in chicks. ISAH Warsaw, Poland, 2005, 2, 60-65.

[129]. Yang, E.S.; Choi, M.J.; Kim, J.H.; Choi, K.S.; Kwon, T.K. Withaferin A enhances radiation-induced apoptosis in Caki cells through induction of reactive oxygen species, Bcl-2 downregulation and Akt inhibition. Chem. Biol. Interact., 2011, 190, 9-15.

[130]. Singh, S.; Veena, K.; Singhal, S.; Singh, N. An Indian herbal immunomodulator: highly effective in the treatment of HIV/AIDS. Proceedings of $6^{\text {th }}$ International Conference on AIDS Asia Pacific, Melbourne, Australia, 2001, 5-10.

[131]. Yu, Y.; Hamza, A.; Zhang, T.; Gu, M.; Zou, P.; Newman, B. Withaferin A targets heat shock protein 90 in pancreatic cancer cells. Biochem. Pharmacol., 2010, 79, 542-551.

[132]. Halder, B.; Singh, S.;, Thakur, S.S. Withania somnifera root extract has potent cytotoxic effect against human malignant melanoma cells. PLOS ONE, 2015, 10(10), e 0141053. 
[133]. Wankhede, S.; Langade, D.; Joshi, K.; Sinha, S.R.; Bhattacharyya, S. Examining the effect of Withania somnifera supplementation on muscle strength and recovery: a randomized controlled trial. J. Int. Soc. Sports Nutr., 2015, 12, 43.

[134]. Chandran, U.; Patwardhan, B. Network ethnopharmacological evaluation of the immunomodulatory activity of Withania somnifera. J. Ethnopharmacol., 2017, 197, 250-256.

[135]. Govindachari, TR. Chemical and biological investigations on Azadirachta indica (the neem tree). Curr. Sci., 1992, 63, 117.

[136]. Alzohairy, M.A. Therapeutics role of Azadirachta indica (Neem) and their active constituents in diseases prevention and treatment. Evid. Based Complement. Alter. Med., 2016, e7382506.

[137]. Gautam, M.K.; Gangwar, M.; Singh, S.K.; Goel, R.K. Effects of Azardirachta indica on vascular endothelial growth factor and cytokines in diabetic deep wound. Planta Med., 2015, 81(9), 713-721.

[138]. Dahiya, P.; Purkayastha, S. Phytochemical screening and antimicrobial activity of some medicinal plants against multi-drug resistant bacteria from clinical isolates. Indian J. Pharm. Sci., 2012, 74, 443-450.

[139]. Bharitkar, Y.P.; Bathini, S.; Ojha, D.; Ghosh, S.; Mukherjee, H.; Kuotsu, K.; Chattopadhyay, D.; Mondal, N.B. Antibacterial and antiviral evaluation of sulfonoquinovosyldiacylglyceride:A glycolipid isolated from Azadirachta indica leaves. Lett. Appl. Microbiol., 2014, 58, 184-189.

[140]. Ravva, S.V.; Korn, A. Effect of Neem (Azadirachta indica) on the Survival of Escherichia coli O157:H7 in Dairy Manure. Int. J. Environ. Res. Public Health, 2015, 12, 7794-7803.

[141]. Thankam, M. Aloe vera. In :Plants and herbs for the treatment of diabetes .Thajema publishers.USA, 2011.

[142]. Somsak, V.; Chachiyo, S.; Jaihan, U.; Nakinchat, S. Protective effect of aqueous crude extract of Neem (Azadirachta indica) leaves on Plasmodium berghei-induced renal damage in mice. J. Trop. Med., 2015, 1-5.

[143]. Vanna, G.S. Miracles of Neem tree. Rosayan Phamacy, New Delhi, 1976.

[144]. Shakti, N.U.; Suman, D.; Sanjay, G.; Talwar, G.P. Immunomodulatory effects of neem (Azadirachta indica) oil. Int. J. Immunopharm., 1992, 14(7), 1187-1193.

[145]. Mulla, M.S.; Su, T. Activity and biological effects of neem products against arthropods of medical and veterinary importance. J Am Mosquito Control Assoc., 1999, 15, 133-152.

[146]. Upadhyay, S.N.; Dhawan, S.; Garg, S.; Talwar, G.P. Immunomodulatory effects of neem (Azadirachta indica) oil. Int J. Immunopharmacol., 1992, 14(7), 1187-1193.

[147]. Ray, A.; Banerjee, B.D.; Sen, P. Modulation of humoral and cell-mediated immune responses by Azadirachta indica (Neem) in mice. Indian J. Exp. Biol., 1996, 34(7), 698-701.

[148]. Sadekar, R.D.; Kolte, A.Y.; Barmase, B.S.; Desai, V.F. Immunopotentiating effects of Azadirachta indica (Neem) dry leaves powder in broilers, naturally infected with IBD virus. Indian J. Exp. Biol., 1998, 36(11), 151-153.

[149]. Baral, R.; Mandal, I.; Chattopadhyay, U. Immunostimulatory neem leaf preparation act as an adjuvant to enhance the efficacy of poorly immunogenic B16 melanoma surface antigen vaccine. Int. Immunopharmacol., 2005, 5, 1343-1352.

[150]. Udeinya, I.J.; Mbah, A.U.; Chijioke, C.P.; Shu, E.N. An antimalarial extract from neem leaves is antiretroviral. Trans. R. Soc. Trop. Med. Hyg., 2004, 98, 435-7.

[151]. Ghosh, D.; Bose, A.; Haque, E.; Baral, R. Pretreatment with neem (Azadirachta indica) leaf preparation in Swiss mice diminishes leukopenia and enhances the antitumor activity ofcyclophosphamide. Phytother. Res., 2006, 20(9), 814818.

[152]. Haque, E.; Mandal, I.; Pal, S.; Baral, R. Prophylactic dose of neem (Azadirachta indica) leaf preparation restricting murine tumor growth is nontoxic, hematostimulatory and immunostimulatory. Immunopharmacol. Immunotoxicol., 2006, 28, 33-50.

[153]. Tiwari, R.; Verma, A.K.; Chakraborty, S.; Dhama, K.; Singh, S.V. Neem (Azadirachta indica) and its Potential for Safeguarding Health of Animals and Humans: A Review. J. Biol. Sci., 2014b, 14, 110-123.

[154]. Nwachukwu, N.; Iweala, E.J. Influence of extraction methods on the hepatotoxicity of Azadirachta indica bark extract on albino rats. Global J. Pure Appl. Sci., 2009, 15, 369-372.

[155]. Deng, Y.X.; Cao, M.; Shi, D.X.; Yin, Z.Q.; Jia, R.Y.; Xu, J.; Wang, C.; Lv, C.; Liang, X.X.; He, C.L.; Yang, Z.R.; Zhao, J. Toxicological evaluation of neem (Azadirachta indica) oil: acute and subacute toxicity. Environ. Toxicol. Pharmacol., 2013, 35(2), 240-246.

[156]. Dhama et al., 2016

[157]. Singh, S.S; Pandey, S.C.; Srivastava, S.; Gupta, V.S.; Patro, B.; Ghosh, A.C. Chemistry and medicinal properties of Tinospora cordifolia (guduchi). Indian J. Pharmacol., 2003, 35, 83-91.

[158]. Kapil, A.; Sharma, S. Immunopotentiating compounds from Tinospora cordifolia. J. Ethnopharmacol., 1997, 58, 8995.

[159]. Saha, S.; Ghosh, S. Tinospora cordifolia: One plant, many roles. Anc. Sci. Life., 2012, 31(4), 151-159.

[160]. Aher, V.D.; Wahi, A. Pharmacological study of Tinospora cordifolia as an immunomodulator. Int. J. Curr. Pharmac. Res., 2010, 2(4), 52-54.

[161]. Narayanan, A.S.; Raja, S.S.; Ponmurugan, K.; Kandekar, S.C.; Maripandi, A. Antibacterial activity of selected medicinal plant against multiple antibiotic resistant uropathogens: A study from Kolli Hills, Tamilnadu, India. Benef. Microbes., 2011, 2, 235-243

[162]. Mittal, J.; Sharma, M.M.; Batra, A. Tinospora cordifolia: a multipurpose medicinal plant - A review. J. Med. Plant Stud., 2014, 2(2), 32-47.

[163]. Sachdeva, H.; Sehgal, R.; Kaur, S. Tinospora cordifolia as a protective and immunomodulatory agent in combination with cisplatin against murine visceral leishmaniasis. Exp. Parasitol., 2014, 137, 53-65.

[164]. Nair, P.K.; Rodriguez, S.; Ramachandran, Alamo, A.; Melnick, S.J.; Escalon, E.; Garcia, P.I.; Jr, Wnuk, S.F. Immunestimulating properties of a novel polysaccharide from the medicinal plant Tinospora cordifolia. Int. Immunopharmacol., 2004, 4, 1645-1659. 
[165]. Ramachandran, C. Immune-stimulating properties of a novel polysaccharide from the medicinal plant Tinospora cordifolia. Int. Immunopharmacol., 2004, 4, 1645-1659.

[166]. Diwanay, S.; Gautam, M.; Patwardhan, B. Cytoprotection and immunomodulation in cancer therapy. Curr Med Chem Anti-Canc. Agents, 2004, 4, 479-490.

[167]. Sudhakaran, D.S.; Srirekha, P.; Devasree, L.D.; Premsingh, S.; Michael, R.D. Immunostimulatory effect of Tinospora cordifolia Miers leaf extract in Oreochromis mossambicus. Indian J. Exp. Biol., 2006, 44(9), 726-732.

[168]. Purandare, H.; Supe, A. Immunomodulatory role of Tinospora cordifolia as an adjuvant in surgical treatment of diabetic foot ulcers: a prospective randomized controlled study. Indian J. Med. Sci., 2007, 61(6), 347-555.

[169]. Hashilkar, N.K.; Patil, P.A.; Bagi, J.G.; Patil, S.Y.; Angadi, N.B. Influence of Tinospora cordifolia on wound healing in wistar rats. Int. J. Basic Clin. Pharmacol., 2016, 5(3), 923-928.

[170]. Mukherjee, R.; De U.K.; Ram, G.C. Evaluation of mammary gland immunity and therapeutic potential of Tinospora cordifolia against bovine subclinical mastitis. Trop Anim Health Prod., 2010, 42(4), 645-651.

[171]. Raghu, R.; Sharma, D.; Ramakrishnan, R.; Khanam, S.; Chintalwar, G.J.; Sainis, K.B. Molecular events in the activation of B cells and macrophages by a non-microbial TLR4 agonist, G1-4A from Tinospora cordifolia. Immunol. Lett., 2009, 24,123(1), 60-71.

[172]. Upadhyaya, R.; Sharma, P.R.; V, Verma Anita, K. Assessment of the multifaceted immunomodulatory potential of the aqueous extract of Tinospora cordifolia. Res. J. Chem. Sci., 2011, 1, 71-79.

[173]. Narkhede, A.N.; Jagtap, S.D.; Kasote, D.M.; Kulkarni, O.P.; Harsulkar, A.M. Comparative immunomodulation potential of Tinospora cordifolia (Willd.) Miers ex Hook. F., Tinospora sinensis (Lour.) Merrill and Tinospora cordifolia growing on Azadirachta indica A. Juss. Indian J. Exp. Biol., 2014, 52(8), 808-13.

[174]. Abiramasundari, G.; Gowda. C.M.; Pampapathi, G.; Praveen, S.; Shivamurugan, S.; Vijaykumar. M.; et al., Ethnomedicine based evaluation of osteoprotective properties of Tinospora cordifolia on in vitro and in vivo model systems. Biomed Pharmacother., 2017, 87, 342-354.

[175]. Subash-Babu, P.; Alshammari, G.M.; Ignacimuthu, S.; Alshatwi, A.A. Epoxy clerodane diterpene inhibits MCF-7 human breast cancer cell growth by regulating the expression of the functional apoptotic genes Cdkn2A, Rb1, mdm2 and p53. Biomed Pharmacother, 2017, 87, 388-396.

[176]. Tierra, M. Echinacea: an effective alternative to antibiotics. J. Herb Pharmacother., 2000, 7(2), 79-89.

[177]. Shah, S.A.; Sander, S.; White, C.M.; Rinaldi, M.; Coleman, C.I. Evaluation of echinacea for the prevention and treatment of the common cold: a meta-analysis. Lancet Infect Dis., 2007, 7(7), 73-80.

[178]. Bauer, R. New knowledge regarding the effect and effectiveness of Echinacea purpurea extract. Wien Med. Wochenschr., 2002, 152, 407-411.

[179]. Block, K.I.; Mead, M.N. Immune system effects of echinacea, ginseng, and astragalus: a review. Integr. Cancer Ther. 2003, 2(3), 247-267.

[180]. Glanville, I. Echinacea: immune effects need more research. Adv. Nurse Pract., 2003, 11(8), 25-26.

[181]. Braunig, B.; Dorn, M.; Knick, E. Echinacea purpurea radix for strengthening the immune response in flu-like infections. Zeitschrift Fur Phytotherapie, 1992, 13, 7-13.

[182]. Schoneberger, D. The influence of immune-stimulating effects of pressed juice from Echinacea purpurea on the course and severity of colds. Forum Immunolog., 1992, 8, 2-12.

[183]. Cichello, S.A.; Yao, Q.; He, X.Q. Proliferative activity of a blend of Echinacea angustifolia and Echinacea purpurea root extracts in human vein epithelial, HeLa, and QBC-939 cell lines, but not in Beas-2b cell lines. J. Trad. Complement. Med., 2016, 6(2), 193-197.

[184]. Ekta, N.; Haria, M.; Ann, D.; Perera, N. David, Senchina, S. Immunomodulatory effects of Echinacea laevigata ethanol tinctures produced from different organs. 2016, doi: 10.1093/biohorizons/hzw001.

[185]. Melchart, D.; Linde, K.; Worku, F.; Sarkady, L.; Holzmann, M.; Jurcic, K.; Wagner, H. Results of five randomized studies on the immunomodulatory activity of preparations of Echinacea. J. Altern. Complement. Med., 1995, 1(2), 145160.

[186]. Jessica Houdret. E.chinacea purpurea .In: Practical Herb Garden Anness publishing Ltd. Hermes House 88-89 Blackfriars Road, London SEI 8HA, 2003b,145.

[187]. Jurkstiene, V.; Kondrotas, A.J.; Kevelaitis, E. Compensatory reactions of immune system and action of Purple Coneflower (Echinacea purpurea) preparations. Medicina (Kaunas), 2004, 40(7), 657-662.

[188]. Wang, C.Y.; Chiao, M.T.; Yen, P.J.; Huang, W.C.; Hou, C.C.; Chien, S.C.; Yeh, K.C.; Yang, W.C.; Shyur, L.F.; Yang, N,S. Modulatory effects of Echinacea purpurea extracts on human dendritic cells: a cell- and gene-based study. Genomics, 2006, 88(6), 801-808.

[189]. Sullivan, A.M.; Laba, J.G.; Moore, J.A.; Lee, T.D. Echinacea-induced macrophage activation. Immunopharmacol. Immunotoxicol., 2008, 30(3), 553-574.

[190]. Farinacci, M.; Colitti, M.; Stefanon, B. Modulation f ovine neutrophil function and apoptosis by standardized extracts of Echinacea angustifolia, Butea frondosa and Curcuma longa. Vet. Immunol. Immunopathol., 2009, 128(4), 366-373.

[191]. Benson, J.M.; Pokorny, A.J.; Rhule, A.; Wenner, C.A.; Kandhi, V.; Cech, N.B.; Shepherd, D.M. Echinacea purpurea extracts modulate murine dendritic cell fate and function. Food Chem. Toxicol., 2010, 48(5), 1170-1177.

[192]. Li, Y.; Wang, Y.; Wu, Y.; Wang, B.; Chen, X.; Xu, X.; Chen, H.; Li, W.; Xu, X. Echinacea pupurea extracts promote murine dendritic cell maturation by activation of JNK, p38 MAPK and NF-кB pathways. Dev. Comp. Immunol., 2017, 73, 21-26.

[193]. Keplinger, K.; Laus, G.; Wurm, M.; Dierich, M.P.; Teppner, H. Uncaria tomentosa (Willd) ethnomedicinal use and new pharmacological, toxicological and botanical results. J. Ethnopharmacol., 1999, 64, 23-34.

[194]. Leslie, T. Uncaria tomentosa (Cats claw) In: The Healing Power of Rainforest Herbs, A guide to understanding and using herbal medicinals. Square one publishers, USA. 2005, 217- 223. 
[195]. Rojas-Duran, R.; González-Aspajo, G.; Ruiz-Martel, C.; Bourdy, G.; Doroteo-Ortega, V.H.; Alban-Castillo, J.; Robert, G.; Auberger, P.; Deharo, E. Anti-inflammatory activity of Mitraphylline isolated from Uncaria tomentosa bark. J. Ethanopharmacol., 2012, 143(3), 801-804.

[196]. Frackowiak, T.; Baczek, T.; Roman, K. Binding of an oxindole alkaloid from Uncaria tomentosa to amyloid protein (Abeta1-40). Z. Naturforsch C., 2006, 61(11-12), 821-826.

[197]. García Prado, E., et al. Antiproliferative effects of mitraphylline, a pentacyclic oxindole alkaloid of Uncaria tomentosa on human glioma and neuroblastoma cell lines. Phytomed., 2007, 14(4), 280-284.

[198]. Eberlin, S.; dos Santos, L.M.; Queiroz, M.L. Uncaria tomentosa extract increases the number of myeloid progenitor cells in the bone marrow of mice infected with Listeria monocytogenes. Int. Immunopharmacol., 2005, 5, 1235-1246.

[199]. Winkler, C. In vitro effects of two extracts and two pure alkaloid preparations of Uncaria tomentosa on peripheral blood mononuclear cells. Planta Med., 2004, 70, 205-210.

[200]. Sandoval-Chacón, M.; Thompson, J.H.; Zhang, X.J.; Liu, X.; Mannick, E.E.; Sadowska-Krowicka, H.; Charbonnet, R.M.; Clark, D.A.; Miller, M.J. Antiinflammatory actions of cat's claw: the role of NF-kappa B. Aliment. Pharmacol. Ther., 1998, 12, 1279-1289.

[201]. Wurm, M.; Kacani, L.; Laus, G.; Keplinger, K.; Dierich, M.P. Pentacyclic oxindole alkaloids from Uncaria tomentosa induce human endothelial cells to release a lymphocyteproliferation- regulating factor. Planta Med., 1998, 64, 701704.

[202]. Sandoval, M.; Charbonnet, R.M.; Okuhama, N.N.; Roberts, J.; Krenova, Z.; Trentacosti, A.M.; Miller, M.J. Cat's claw inhibits TNF-alpha production and scavenges free radicals: role in cytoprotection. Free Radic. Biol. Med., 2000, 29, 71-78.

[203]. Mur, E.; Hartig, F.; Eibl, G.; Schirmer, M. Randomized double-blind trial of an extract from the pentacyclic alkaloidchemotype of Uncaria tomentosa for the treatment of rheumatoid arthritis. J Rheumatol., 2002, 29, 678-681.

[204]. Herrera, D.R.; Durand-Ramirez, J.E.; Falcão, A.; Silva, E.J.; Santos, E.B.; Gomes, B.P. Antimicrobial activity and substantivity of Uncaria tomentosa in infected root canal dentin. Braz. Oral Res., 2016, 30(1), e61.

[205]. Reis, S.R.; Valente, L.M.; Sampaio, A.L.; Siani, A.C.; Gandini, M.; Azeredo, E.L.; D'Avila, L.A.; Mazzei, J.L.; Henriques, M,G.; Kubelka, C.F. Immunomodulating and antiviral activities of Uncaria tomentosa on human monocytes infected with Dengue Virus-2. Int. Immunopharmacol., 2008, 8(3), 468-476.

[206]. Castillo, G.; Snow, A.D. Pharmaceutical composition containing Uncaria tomentosaextract for treating Alzhemer's disease and other amyloidosis. Patent-Pct. Int. Paol., 1998, 33, 659-666.

[207]. Muhammad, I.; Dunbar, D.C.; Khan, R.A.; Ganzera, M.; Khan, I.A. Investigation of Una de Gato 1. 7-Deoxyloganic acid and 15N NMR spectroscopic studies on penta cyclic oxindole alkaloids from Uncaria tomentosa. Phytochem., 2001, 57(5), 781-785.

[208]. Mohamed, A. F; Matsumoto, K.; Tabata, K.; Takayama, H.; Kitajima, M.; Watanabe, H. Effects of Uncaria tomentosa total alkaloids and its components on experimental amnesia in mice: elucidation using the passive avoidance test. $J$. Pharm. Pharmacol., 2000, 52 (12), 1553-1561.

[209]. Roth, B.L.; Shapiro, D.A. Insights into the structure and function, of 5-HT(2) family serotonin receptors reveal novel strategies for therapeutic target drvelopment. Expert. Opin. Ther. Targets, 2001, 5, 685-695.

[210]. Kang, T.H.; Matsumoto, K.; Tohda, M.; Murakami, Y.; Takayama, H.; Kitajima, M.; Aimi, N.; Watanabe, H. Pteropodine and isopteropodine positively modulate the function of rat musacarinic M(1)and 5- HT(2) receptors expressed in Xenopus oocyte. Eur. J. Pharmacol., 2002, 444(1-2), 39-45.

[211]. Falkiewicz, B.; Lukasiak, J. Cat's claw Uncaria tomentosa (Willd.) DC. and Uncaria guianensis (Aublet) Gmell.) - a review of published scientific literature. Case Rep. Clin. Pract. Rev., 2001, 2, 305-316.

[212]. Pilarski, R.; Bednarczyk, M.; Gulewicz, K. Evaluation of biological activity of Uncaria tomentosa (Willd.) DC. using the chicken embryo model. Folia biologica (Krakow). 2009, 57(3-4), 207-212.

[213]. Gupta, S.K.; Prakash, J.; Srivastava, S. Validation of traditional claim of Tulsi, Ocimum sanctum Linn as a medicinal plant. Indian J. Exp. Biol., 2002, 40(7), 765-773.

[214]. Kumar, A.; Rahal, A.; Chakraborty, S.; Tiwari, R.; Latheef, S.K.; Dhama, K. Ocimum sanctum (Tulsi): a miracle herb and boon to medical science - A Review. Int. J. Agr. Plant Prod., 2013, 4(7), 1580-1589.

[215]. Jain, S.; Mohan, R.; Rai, R. Ocimum sanctum as an herbal medicine: A Review. Int. J. Maxillofacial Res., 2015, 1(1), 3-12.

[216]. Chattopadhya, R.R.; Sarkar, S.K.; Ganguly, S.; Medda, C.; Basu, T.K. Hepatoprotective activity of Ocimum sanctum leaf extract against paracetamol induced hepatic damage in rats. Indian. J. Pharmacol., 1992, 24, 163-165.

[217]. Lukmanul, H.F.; Gowri, S.C.; Girija, S. Chemical composition and antioxidant property of holy basil (Ocimum sanctum l.) leaves, stems, and inflorescence and their in vitro callus cultures. J. Agric. Food Chem.; 2007, 55(22), 9109-9117.

[218]. Rajeshwari, S. Ocimum Sanctum the Indian Home Remedy. In: Current Medicinal Scene, Rajeswari Foundations Limited, Bombay. 1992.

[219]. Singh, S.; Majumdar, D.K.; Rehan, H.M.S. Evaluation of anti-inflammatory potential of fixed oil of Ocimum sanctum (Holybasil) and its possible mechanism of action. J. Ethnopharmacol., 1996, 54, 19-26.

[220]. Sen, P. Therapeutic potentials of Tulsi : from experience to facts. Drugs News \& Views., 1993, 1(2), $15-21$.

[221]. Devi, P.U.; Ganasoundari, A. Radioprotective effect of leaf extract of Indian medicinal plant Ocimum sanctum. Indian J. Exp. Biol., 1995, 33, 205-208.

[222]. Mukherjee, R.; Dash, P.K.; Ram, G.C. Immunotherapeutic potential of Ocimum sanctum (L) in bovine subclinical mastitis. Research in veterinary science., 2005, 79, 37-43. 
[223]. Kumar, K.P.S.; Bhowmik, D.; Dutta, A.; Yadav, A.P.; Paswan, S.; Srivastava, S.; Deb, L. Recent trends in potential traditional indian herbs emblica officinalis and its medicinal importance. J. Pharmacog. Phytochem., 2012b, 1(1), 2432 .

[224]. Manaharan, T.; Thirugnanasampandan, R.; Jayakumar, R.; Kanthimathi, M.S.; Ramya, G.; Ramnath, M.G. Purified essential oil from Ocimum sanctum Linn. triggers the apoptotic mechanism in human breast cancer cells. Pharmacogn. Mag., 2016, 12(Suppl 3), S327-31.

[225]. Hemapriya, V.S. Immunostimulatory effect of leaf extracts of few medicinal plants in Oreochromis mossambicus (Peters), MSc thesis-herbivores and humans. Natural Products from Plants, CRC Press, 1997, 478.

[226]. Logambal, S.M.; Venkalalakshmi, S.; Michael, R.D. Immunostimulatory effect of leaf extract of Ocimum sanctum Linn in Oreochromis mossambicus (Peters). Hydrobiolog., 2000, 430, 113-120.

[227]. Mediratta, P.K.; Sharma, K.K.; Singh, S. Evaluation of immunomodulatory potential of Ocimum sanctum seed oil and its possible mechanism of action. J. Ethnopharmacol., 2002, 80(1), 15-20.

[228]. Harikrishnan, R.; Balasundaram, C. In vitro and in vivo studies of the use of some medicinal herbals against the pathogen Aeromonas hydrophila in goldfish. J. Aquat. Anim. Health, 2008, 20, 165-176.

[229]. Mishra, P.; Mishra, S. Study of antibacterial activity of Ocimum sanctum extract against gram positive and gram negative bacteria. Am. J. Food Tech., 2011, 6, 336-341.

[230]. Nahak, G.; Sahu, R.K. Immunostimulatory Effect Of Ocimum Sanctum Linn. Leaf Extract In Clarias Batrachus Linn. Asian J. Pharm. Clin. Res., 2014, 7(3), 157-163.

[231]. Das, R.; Raman, R.P.; Saha, H.; Singh, R. Effect of Ocimum sanctum Linn. (Tulsi) extract on the immunity and survival of Labeo rohita (Hamilton) infected with Aeromonas hydrophila. Aquaculture Res., 2015, 46(5), 1111-1121.

[232]. Araujo, C.A.C.; Leon, L.L. Biological activities of Curcuma longa L. Mem. Inst. Oswaldo Cruz., 2001, 96, 723-728.

[233]. Castleman, M. Turmeric (Curcuma longa) In: The new healing herbs Rondale Inc. USA, 2009b, 461-465.

[234]. Srimal, R.C. Turmeric: a brief review of medicinal properties. Fitoterapia, 1997, 68, 483-493.

[235]. Bagad, A.S.; Joseph, J.A.; Bhaskaran, N. and Agarwal, A. Comparative evaluation of anti-inflammatory activity of curcuminoids, turmerones, and aqueous extract of Curcuma longa. Adv. Pharmacol. Sci., 2013. e805756.

[236]. Reddy, P. H.; Manczak, M.; Yin, X.; Grady, M. C.; Mitchell, A.; Kandimalla, R.; Kuruva, C.S. Protective effects of a natural product, curcumin, against amyloid $\beta$ induced mitochondrial and synaptic toxicities in Alzheimer's disease. $J$. Invest. Med., 2016, 64(8), 1220-1234.

[237]. ElHage. S.; Mathew, T. Turmeric.Curcuma longa.In: Advances in Medical and Veterinary Virology, Immunology and Epidemiology Vol.5. Benefits of herbs and spices in food. 2005a, 73-75.

[238]. Chattopadhyay, I.; Biswas, K.; Bandyopadhyay, U.; Banerjee, R.K. Turmeric and curcumin: Biological actions and medicinal applications. Curr. Sci., 2004, 87(1), 44-53.

[239]. Menon, L.G.; Kuttan, R.; Kuttan, G. Inhibition of lung metastasis in mice induced by B16F10 melanom cell by polyphenolic compounds. Cancer Lett., 1995, 95, 221-225.

[240]. Samaha, H.S.; Kelloff, G.J.; Steele, V.; Rao, C.V.; Reddy, B.S. Modulation of apoptosis by sulindac curcumin, phenylthexy isothiocynate: apoptotic index as biomarker in colon cancer. Chemoprevention and promotion. Cancer Res., 1997, 57, 1301-1305.

[241]. Stengler, M.; Turmeric. In: The natural Physician' Healing Therapies Proven Remedies That Doctors Don't Know about. Prentice Hall press, Bottomline books edition. USA. 2001, 444- 446.

[242]. Farag, M.R.; Alagawany, M.; Dhama, K. Antidotal effect of turmeric (Curcuma longa) against endosulfan-induced cytogenotoxicity and immunotoxicity in broiler chicks. Intern. J. Pharmacol., 2014, 10(8), 429-439.

[243]. Alagawany, M.; Farag, M. R.; Dhama, K. Nutritional and biological effects of turmeric (Curcuma longa) supplementation on performance, serum biochemical parameters and oxidative status of broiler chicks exposed to endosulfan in the diets. Asian J. Anim. Sci. Vet. Adv., 2015c, 10(2), 86-96.

[244]. Motterlini , R.; Foresti , R.; Bassi, R.; Green, C.J. Curcumin, an antioxidant and anti-inflammatory agent, induces heme oxygenase-1 and protects endothelial cells against oxidative stress. Free Radic. Biol. Med., 2000, 28, 13031312 .

[245]. Kang, B.Y.; Song, Y.J.; Kim, K.M.; Choe, Y.K.; Hwang, S.Y.; Kim, T.S. Curcumin inhibits Th1 cytokine profile in CD4+ T cells by suppressing interleukin-12 production in macrophages. Br. J. Pharmacol., 1999, 128, 380-384.

[246]. Joe, B.; Lokesh, B.R. Dietary n-3 fatty acids, curcumin and capsaicin lower the release of lysosomal enzymes and eicosanoids in rat peritoneal macrophages. Mol. Cell Biochem. 2000, 203(1-2), 153-61.

[247]. Churchill, M.; Chadburn, A.; Bilinski, R.T.; Bertagnolli, M.M. Inhibition of intestinal tumors by curcumin is associated with changes in the intestinal immune cell profile. J. Surg. Res., 2000, 89, 169-175.

[248]. Yasni, S.; Yoshiie, K.; Oda, H.; Sugano, M.; Lniazumi, K. Dietary Curcum xanthorrhiza Roxb increases mitogenic response of splenic lymphocytes in rats and alters population of lymphocytes in mice. J. Nutri. Sci. Vitaminol., 1993, 39, 345-354.

[249]. Inagawa, H.; Nishizawa, T.; Tsukioka, D.; Suda, T.; Chiba, Y.; Okutomi, T.; Morikawa, A.; Soma, G.I.; Mizuno, D.I. Homeostasis as regulated by activated macrophages. LPs of plant origin other than wheat flour and their concomitant bacteria. Chem. Pharm. Bull., 1992, 40, 994-997.

[250]. South, E.H.; Exon, J.H.; Hendrix, K. Dietary curcumin enhances antibody response in mice. Immunopharmocol. Immunotoxicol., 1997, 19, 105-119.

[251]. Bansal, S.; Chhibber, S. Curcumin alone and in combination with augmentin protects against pulmonary inflammation and acute lung injury generated during Klebsiella pneumoniae B5055-induced lung infection in BALB/c mice. J Med. Microbiol., 2010, 59(4), 429-437. 
[252]. Witkin, J.M.; Li, X. Curcumin, an active constiuent of the ancient medicinal herb Curcuma longa L.: some uses and the establishment and biological basis of medical efficacy. CNS Neurolog. Disord. Drug Targets, 2013, 12(4), 487497.

[253]. Balaji, B.; Chempakam, B. Pharmacokinetics prediction and drugability assessment of diphenyl-heptanoids from turmeric (Curcuma longa $L$ ). Med. Chem., 2015, 5(2), 130-138.

[254]. Schneider, T.P.; McKinnon, M.J.; Brown, S.; Togni, B.M. Comparative absorption of a standardized curcuminoid mixture and its lecithin formulation. J. Nat. Prod., 2011, 74, 664-669.

[255]. Krishnakumar, I.M.; Ravi, A.; Kumar, D.; Kuttan, R.; Maliakel, B. An enhanced bioavailable formulation of curcumin using fenugreek derived soluble dietary fibre. J. Funct. Foods, 2012, 4, 348-357.

[256]. Goel, A.; Kunnumakkara, A.B.; Aggarwal, B.B. Curcumin as "Curecumin": from kitchen to clinic. Biochem. Pharmacol., 2008, 75, 787-809.

[257]. Park, E.J.; Pizzuto, J.M. Botanicals in cancer chemoprevention. Cancer Met. Rev., 2002, 21, 231-255.

[258]. Altman, R.D.; Marcussen, K.C. Effects of a ginger extract on knee pain in patients with osteoarthritis. Arthritis and Rheumatism, 2001, 44, 2531-2538.

[259]. Grant, K.L.; Lutz, R.B. Alternative therapies: ginger. Am. J. Health-Syst. Pharm., 2000, 57, 945-947.

[260]. Aggarwal, B.B.; Shishodia, S. Suppression of the nuclear factor-kappaB activation pathway by spice-derived phytochemicals: reasoning for seasoning. Ann. New York Acad. Sci., 2004, 1030, 434-441.

[261]. Aggarwal, B.B; Prasad, S.; Reuter, S.; Kannappan, R.; Yadev, V.R.; Park, B.; Kim, J.H.; Gupta, S.C.; Phromnoi, K.; Sundaram, C.; Prasad, S.; Chaturvedi, M.M.; Sung, B. Identification of Novel Anti-inflammatory Agents from Ayurvedic Medicine for Prevention of Chronic Diseases "Reverse Pharmacology" and "Bedside to Bench" Approach. Curr. Drug Targets. 2011, 12(11), 1595-1653.

[262]. Grzanna, R.; Lindmark, L.; Frondoza, C.G. Ginger - an herbal medicinal product with broad anti-inflammatory actions. J. Med. Food., 2005, 8(2), 125-132.

[263]. Shukla, Y.; Singh, M. Cancer preventive properties of ginger: a brief review. Food Chem. Toxicol., 2007, 45(5), 683690.

[264]. Ding, G.H.; Naora, K.; Hayashibara, M.; Katagiri, Y.; Kano, Y.; Iwamoto, K. Pharmacokinetics of [6]-gingerol after intravenous administration in rats. Chem. Pharm. Bull. (Tokyo), 1991, 39(6), 1612-1614.

[265]. Zick, S.M.; Djuric, Z.; Ruffin, M.T.; Litzinger, A.J.; Normolle, D.P.; Feng, M.R.; Brenner, D.E. Pharmacokinetics of 6-, 8-, 10-Gingerols and 6-Shogaol and conjugate metabolites in healthy human subjects. Cancer Epidemiol. Biomarkers Prev., 2008, 17(8), 1930-1936.

[266]. Serthe, J.A.; Basile, A.C.; Oshioo, T.T.; Silva, F.D.; Mazella, A.A. Preventive anti-ulcer activity of the rhizome extract of Zingiber officinale. Fitoterapia., 1991, 8(1), 55-59.

[267]. Raji, Y.; Udoh, U.S.; Oluwadara, O.; Oakinsomisoye, O.; Sawobajo, O.; Adeshoga, K. Anti-inflammatory and analgesic properties of the rhizome extract of zingiber officinale. Afr. J. Biomed. Res., 2002, 5, 121-124.

[268]. Stengler, M. Ginger Root. In: The natural physician's healing therapies. Bottomline books USA. 2005b, $207-211$.

[269]. Castleman, M. Ginger. In: The new healing herbs, Third Ed.Rondale Inc.USA, 2009c, 239-243.

[270]. Jafarzadeh, A.; Ahangar-Parvin, R.; Nemat, M.; Taghipour, Z.; Shamsizadeh, A.; Ayoobi, F.; Hassan, Z.M. Ginger extract modulates the expression of IL-12 and TGF- $\beta$ in the central nervous system and serum of mice with experimental autoimmune encephalomyelitis. Avicenna J. Phytomed., 2017, 7(1), 54-65.

[271]. Agarwal, M.; Walia, S.; Dhingra, S.; Khambay, B.P.S. Insect growth inhibition, antifeedant and antifungal activity of compounds isolated/derived from Zingiber officinale Roscoe (ginger) rhizomes. Pest Manag. Sci., 2001, 57(3), 289300.

[272]. Kristen, G.; Yue, H.; Dawn, A.L.; Susan, L.P.; Bolanle, A.A.; Gail, B.M. Standardized ginger (Zingiber officinale) extract reduces bacterial load and suppresses acute and chronic inflammation in Mongolian gerbils infected with cagA +Helicobacter pylori. Pharm. Biol., 2009, 47(1), 92-98.

[273]. Habibi, R.; Sadeghi, G.; Karimi, A. Effect of different concentrations of ginger root powder and its essential oil on growth performance, serum metabolites and antioxidant status in broiler chicks under heat stress. Br. Poult. Sci., 2014, 55(2), 228-37. doi: 10.1080/00071668.2014.887830. Epub 2014 Apr 24.

[274]. Karangiya, V.K.; Savsani, H.H.; Patil, S.S.; Garg, D.D.; Murthy, K.S.; Ribadiya, N.K.; Vekariya, S.J. Effect of dietary supplementation of garlic, ginger and their combination on feed intake, growth performance and economics in commercial broilers. Vet. World, 2016, 9(3), 245-250.

[275]. Nicoll, R.; Henein, M.Y. Ginger (Zingiber officinale Roscoe): a hot remedy for cardiovascular disease? Int. J. Cardiol., 2009, 131(3), 408-409.

[276]. Arulvasu, C.A.; Mania, K.; · Chandhirasekara, D.; · Prabhua, D.; Sivagnanam, S. Effect of dietary administration of Zingiber officinale on growth, survival and immune response of Indian major carp, Catla catla (Ham.) Inter. J. Pharm. Pharmaceut. Sci., 2013, 5(3), 108-115.

[277]. Ghasemi, H.A.; Taherpour, K. Comparison of broiler performance, blood biochemistry, hematology and immune response when feed diets were supplemented with ginger essential oils or mannan-oligosaccharide. Iranian J. Vet. Med., 2015, 9(3), 195-205.

[278]. Kapoor, D.; Trikha, D.; Vijayvergiya, R.; Parashar, K.K.; Kaul, D.; Dhawan, V. Short-term adjuvant therapy with terminalia arjuna attenuates ongoing inflammation and immune imbalance in patients with stable coronary artery disease: in vitro and in vivo evidence. J. Cardiovasc. Transl. Res., 2015, 8(3), 173-86.

[279]. Ayaz, E.; Alpsoy, H.C. Garlic (Allium sativum) and Traditional Medicine. Turkish J. Parasitol., 2007, 31, 145-149.

[280]. Newall, C.A.; Anderson, L.A.; Phillipson, J.D. Herbal medicines: a guide for health-care professionals. London: Pharmaceutical Press., 1996, 296. 
[281]. Castleman, M. Garlic In: The healing herbs. The ultimate guide to the curative power of nature'smedicines.Rondale press Pa USA, 1991, 177-182.

[282]. ElHage, S.; Mathew, T. Garlic .In: Advances in Medical and Veterinary Virology, Immunology and Epidemiology Vol.5. Benefits of herbs and spices in food. 2005b., 39-43.

[283]. EL-mahmood, M.A. Efficacy of crude extracts of garlic (Allium sativum Linn) against nosocomial Escherichia coli, Staphylococcus aureus, Streptococcus pneumoniea and Pseudomonas aeruginosa. J. Med. Plants Res., 2009, 3, 179185.

[284]. Weber, N.D.; Andersen, D.O.; North, J.A.; Murray, B.K.; Lawson, L.D.; Hughes, B.G. In vitro virucidal effects of Allium sativum (garlic) extract and compounds. Planta Med., 1992, 58, 417-423.

[285]. Mikaili, P.; Maadirad, S.; Moloudizargari, M.; Aghajanshakeri, S.; Sarahroodi, S. Therapeutic uses and pharmacological properties of Garlic, Shallot, and their biologically active compounds. Iran J. Basic Med. Sci., 2013, 16(10), 1031-1048.

[286]. Ledezma, E.; Apitz-Castro, R. Ajoene the main active compound of garlic (Allium sativum): a new antifungal agent. Rev. Iberoam Micol., 2006, 23, 75-80.

[287]. Kamel, R.O.; El-Shinnawy, N.A. Immunomodulatory effect of garlic oil extract on Schistosoma mansoni infected mice. Asian Pac. J. Trop. Med., 2015, 8(12), 999-1005. doi: 10.1016/j.apjtm.2015.11.016. Epub 2015 Nov 14.

[288]. Ried, K. Garlic lowers blood pressure in hypertensive individuals, regulates serum cholesterol, and stimulates immunity: An updated meta-analysis and review. J. Nutr., 2016, 146(2), 389S-96S. doi: 10.3945/jn.114.202192. Epub 2016 Jan 13.

[289]. Lau, B.H.; Yamasaki, T.; Gridley, D.S. Garlic compounds modulate macrophage and T lymphocyte functions. Mol. Biother., 1991, 3, 103-107.

[290]. Morioka, N.; Sze, L.L.; Morton, D.L.; Irie, R.F. A protein fraction from aged garlic extract enhances cytotoxicity and proliferation of human lymphocytes mediated by interleukin-2 and concanavalin A. Cancer Immunol. Immunother., 1993, 37, 316-322.

[291]. Eikai, K.; Naoto, U.; Shigeo, K.; Yoichi, I. Immunomodulatory effects of aged garlic extract. J. Nutr., 2001, 131, 1075-1079.

[292]. Clement, F.; Pramod, S.N.; Venkatesh, Y.P. Identity of the immunomodulatory proteins from garlic (Allium sativum) with the major garlic lectins or agglutinins. Int. Immunopharmacol., 2010, 10(3), 316-324.

[293]. Percival, S.S. Aged garlic extract modifies human immunity. J. Nutr., 2016, 146(2), 433S-6S. doi: 10.3945/jn.115.210427. Epub 2016 Jan 13.

[294]. Elhalwagy, M.E.; Darwish, N.S.; Shokry, D.A.; El-Aal, A.G.; Abd-Alrahman, S.H.; Nahas, A.A.; Ziada, R.M. Garlic and alpha lipoic supplementation enhance the immune system of albino rats and alleviate implications of pesticides mixtures. Int. J. Clin. Exp. Med., 2015, 8(5), 7689-700.

[295]. Sheoran, N.; Kumar, R.; Kumar, A.; Batra, K.; Sihag, S.; Maan, S.; Maan, N.S. Nutrigenomic evaluation of garlic (Allium sativum) and holy basil (Ocimum sanctum) leaf powder supplementation on growth performance and immune characteristics in broilers. Vet. World., 2017, 10(1), 121-129.

[296]. Abd El-Hack, M.E.; Alagawany, M.; Farag, M.R.; Tiwari, R.; Karthik, K.; Dhama, K.; Nutritional, healthical and therapeutic efficacy of Black Cumin (Nigella sativa) in animals, poultry and humans. Inter. J. Pharmacol., 2016a, 12, 232-248.

[297]. Nickavar, B.; Mojab, F.; Javidnia, K.; Amoli, M.A. Chemical composition of the fixed and volatile oils of Nigella sativa L. from Iran. Z. Naturforsch. C., 2003, 58(9-10), 629-631.

[298]. Hajhashemi, V.; Ghannadi, A.; Jafarabadi, H. Black cumin seed essential oil, as a potent analgesic and antiinflammatory drug. Phytother. Res., 2004, 18(3), 195-199.

[299]. Haq, A.; Lobo, P.I.; Al-Tufail, M.; Rama, N.R.; Al-Sedairy, S. Immunomodulatory effect of Nigella sativa proteins fractionated by ion exchange chromatography. Int. J. Immunopharmacol., 1999, 21, 283-295.

[300]. Salem, M.L. Immunomodulatory and therapeutic properties of the Nigella sativa L. seed. Int. Immunopharmacol., 2005, $5,1749-1770$.

[301]. Janfaza, S.; Janfaza, E. The study of pharmacologic and medicinal valuation of thymoquinone of oil of Nigella sativa in the treatment of diseases. Ann. Biol. Res., 2012, 3, 1953-1957.

[302]. Majdalawieh, A.F.; Fayyad, M.W. Immunomodulatory and anti-inflammatory action of Nigella sativa and thymoquinone: A comprehensive review. Int. Immunopharmacol., 2015, 28, 295-304.

[303]. AL-Hothaify, S.A.; Al-Sanabani, M.A. The effects of supplementation Nigella sativa seeds as a natural substance on growth rate, some serum indices, carcass quality and antibody titers of broiler birds. American J. Res. Comm., 2016, $4(3), 43-51$.

[304]. Vaillancourt, F.; Silva, P.; Shi, Q.; Fahmi, H.; Fernandes, J.C.; Benderdour, M. Elucidation of molecular mechanisms underlying the protective effects of thymoquinone against rheumatoid arthritis. J. Cell. Biochem., 2011, 112, $107-117$.

[305]. Mutabagani, A.; El-Mahdy, S.A.M. A study of the anti-inflammatory activity of Nigella sativa L. and thymoquinone in rats. Saudi Pharmaceut. J., 1997, 5, 110-113.

[306]. Mbarek, L.A.; Mouse, H.A.; Elabbadi, N.; Bensalah, M.; Gamouh, A.; et al., Anti-tumor properties of blackseed (Nigella sativa L.) extracts. Braz. J. Med. Biol. Res., 2007, 40, 839-847.

[307]. Majdalawieh, A.F.; Hmaidan, R.; Carr, R.I. Nigella sativa modulates splenocyte proliferation, Th1/Th2 cytokine profile, macrophage function and NK anti-tumor activity. J. Ethnopharmacol., 2010, 131, 268-275.

[308]. Majdalawieh, A.F.; Fayyad, M.W. Recent advances on the anti-cancer properties of Nigella sativa, a widely used food additive. J. Ayur. Integr. Med., 2016, 7(3), 173-180.

[309]. Akhtar, M.S.; Nasir, Z.; Abid, A.R. Effect of feeding powdered Nigella sativa L. seeds on poultry egg production and their suitability for human consumption. Veterinarski Arhiv., 2003, 73, 181-190. 
[310]. Mansour, M.A.; Nagi, M.N.; El-Khatib, A.S.; Al-Bekairi, A.M. Effects of thymoquinone on antioxidant enzyme activities, lipid peroxidation and DT-diaphorase in different tissues of mice: A possible mechanism of action. Cell Biochem. Funct., 2002, 20, 143-151.

[311]. Al Jabre, S.; Al Akloby, O.M.; Al Qurashi, A.R.; Al Dossary, A.; Akhtar, N.; Randhawa, M.A. Thymoquinone, an active principle of Nigella sativa, inhibited Aspergillus niger. Pak. J. Med. Res., 2003, 42, 102-104.

[312]. Arslan, S.O.; Gelir, E.; Armutcu, F.; Coskun, O.; Gurel, A.; Sayan, H.; Celik, I.L. The protective effect of thymoquinone on ethanol-induced acute gastric damage in the rat. Nutr. Res., 2005, 25, 673-680.

[313]. Al-Saleh, I.A.; Billedo, G.; El-Doush, I.I. Levels of selenium, DL-"-tocopherol, DL-(-tocopherol, all-trans-retinol, thymoquinone and thymol in different brands of Nigella sativa seeds. J. Food Compos. Anal., 2006, 19, 167-175.

[314]. Ravindran, J.; Nair, H.B. ; Sung, B.; Prasad, S.; Tekmal, R.R.; Aggarwal, B.B. Thymoquinone poly (lactide-coglycolide) nanoparticles exhibit enhanced anti-proliferative, anti-inflammatory and chemosensitization potential. Biochem. Pharmacol., 2010, 79, 1640-1647.

[315]. Toghyani, M.; Toghyani, M.; Gheisari, A.; Ghalamkari, G.; Mohammadrezaei, M. Growth performance, serum biochemistry and blood hematology of broiler chicks fed different levels of black seed (Nigella sativa ) and peppermint (Mentha piperita ). Livestock Sci., 2010, 129, 173-178.

[316]. Al-Beitawi, N.A.; El-Ghousein, S.S.; Nofal, A.H. Replacing bacitracin methylene disalicylate by crushed Nigella sativa seeds in broiler rations and its effects on growth, blood constituents and immunity. Livestock Sci., 2009, 125, 304-307.

[317]. El-Kadi, A.; Kandil, O. Effect of Nigella sativa (the black seed) on immunity. Bull. Islamic Med., 1986, 4, $344-348$.

[318]. Salomi, M.J.; Nair, S.C.; Panikkar, K.R. Inhibitory effects of Nigella sativa and saffron (Crocus sativus ) on chemical carcinogenesis in mice. Nutr. Cancer, 1991, 16, 67-72.

[319]. Iddamaldeniya, S.S.; Wickramasinghe, N.; Thabrew, I.; Ratnatunge, N.; Thammitiyagodage, M.G. Protection against diethylnitrosoamine-induced hepatocarcinogenesis by an indigenous medicine comprised of Nigella sativa, Hemidesmus indicus and Smilax glabra : A preliminary study. J. Carcinog., 2003, 2(1), 6. Doi: 10.1186/1477-3163-26.

[320]. Musa, D.; Dilsiz, N.; Gumushan, H.; Ulakoglu, G.; Bitiren, M. Antitumor activity of an ethanol extract of Nigella sativa seeds. Biol. Bratis., 2004, 59, 735-740.

[321]. Randhawa, M.A.; Alghamdi, M.S. Anticancer activity of Nigella sativa (black seed)- a review. Am. J. Chin. Med., 2011, 39, 1075-1091.

[322]. Gali-Muhtasib, H.; Diab-Assaf, M.; Boltze, C.; Al-Hmaira, J.; Hartig, R.; Roessner, A.; Schneider-Stock, R. Thymoquinone extracted from black seed triggers apoptotic cell death in human colorectal cancer cells via a p53dependent mechanism. Int. J. Oncol., 2004, 25, 857-866.

[323]. Woo, C.C.; Kumar, A.P.; Sethi, G.; Tan, K.H.B. Thymoquinone: Potential cure for inflammatory disorders and cancer. Biochem. Pharmacol., 2012, 83, 443-451.

[324]. El-Dakhakhny, M.; Barakat, M.; El-Halim, M.A.; SAly, M. Effects of Nigella sativa oil on gastric secretion and ethanol induced ulcer in rats. J. Ethnopharmacol., 2000, 72, 299-304.

[325]. Al-Mofleh, I.A.; Alhaider, A.A.; Mossa, J.S.; Al-Sohaibani, M.O.; Al-Yahya, M.A.; Rafatullah, S.; Shaik, S.A. Gastroprotective effect of an aqueous suspension of black cumin Nigella sativa on necrotizing agents-induced gastric injury in experimental animals. Saudi J. Gastroenterol., 2008, 14, 128-134.

[326]. Alenzi, F.Q.; El-Bolkiny, Y.S.; Salem, M.L.; Protective effects of Nigella sativa oil and thymoquinone against toxicity induced by the anticancer drug cyclophosphamide. Br. J. Biomed. Sci., 2010, 67, 20-28.

[327]. Agbaria, R.; Gabarin, A.; Dahan, A.; Ben-Shabat, S. Anticancer activity of Nigella sativa (black seed) and its relationship with the thermal processing and quinone composition of the seed. Drug Des. Dev. Ther., 2015, 9, 31193124.

[328]. Paranagama, P.A.; Wimalasena, S.; Jayatilake, G.S.; Jayawardena, A.L.; Senanayake, U.M.; Mubarak, A.M. A comparison of essential oil constituents of bark, leaf root and fruit of cinnamon (Cinnamomum zeylanicum Blum), grown in Sri Lanka. J. Natl. Sci. Found. Sri., 2010, 29, 147-153.

[329]. Ranasinghe, P.; Pigera, S.; Premakumara, G.A.; Galappaththy, P.; Constantine, G.R.; Katulanda, P. Medicinal properties of 'true' cinnamon (Cinnamomum zeylanicum): a systematic review. BMC Complement. Alter. Med., 2013, $13,275$.

[330]. Abo El-Maaty, Hayam, M.A.; Rabie, M.H.; El-Khateeb, A.Y. Response of heat-stressed broiler chicks to dietary supplementation with some commercial herbs. Asian J. Anim. Vet. Adv., 2014, 9(12), 743-755.

[331]. Shen, Q.; Chen, F.; Luo, J. Comparison studies on chemical constituents of essential oil from ramulus cinnamomi and cortex cinnamomi by GC-MS. Zhong Yao Cai., 2002, 25, 257-258.

[332]. Rao, P.V.; Gan, S.H. Cinnamon: a multifaceted medicinal plant. Evid. Based Complement. Alternat. Med., 2014, e642942.

[333]. Gruenwald, J.; Freder, J.; Armbruester, N. Cinnamon and health. Crit. Rev. Food Sci. Nutr., 2010, 50, 822-834.

[334]. Naderi, M.; Akbari, M.R.; Asadi-Khoshoei, E.; Khaksar, K.; Khajali, F. Effects of Dietary Inclusion of Turmeric (Curcuma longa) and Cinnamon (Cinnamomum verum) Powders on Performance, Organs Relative Weight and Some Immune System Parameters in Broiler Chickens. Poultry Sci. J., 2014, 2(2), 153-163.

[335]. Symeon, G.K.; Athanasiou, A.; Lykos, N.; Charismiadou, M.A.; Goliomytis, M.; Demiris, N.; Ayoutanti, A.; Simitzis, P.E.; Deligeorgis, S.G. The effects of dietary cinnamon (cinnamomum zeylanicum) oil supplementation on broiler feeding behaviour, growth performance, carcass traits and meat quality characteristics. Ann. Anim. Sci., 2014, 14(4), 883-895.

[336]. Tanaka, N.; Sekiya, N.; Hattori, M.; Goto, H.; Shibahara, N.; Shimada, Y.; Terasawa, K. Measurement of plasma procyanidin B-2 and procyanidin B-3 levels after oral administration in rat. Phytomed., 2003, 10(2-3), 122-126. 
[337]. Chen, Y.; Ma, Y.; Ma, W. Pharmacokinetics and bioavailability of cinnamic acid after oral administration of Ramulus cinnamomi in rats. Eur. J. Drug Metab. Pharmacokinet., 2009, 34(1), 51-56.

[338]. Wondrak, G.T.; Cabello, C.M.; Villeneuve, N.F.; Zhang, S.; Ley, S.; Li, Y.; Sun, Z.; Zhang, D.D. Cinnamoyl-based Nrf2-activators targeting human skin cell photo-oxidative stress. Free Radic. Biol. Med., 2008, 45(4), 385-395.

[339]. Samarasekera, R.; Kalhari, K.S.; Weerasinghe, I.S. Mosquitocidal activity of leaf and bark essential oils of ceylon cinnamomum zeylanicum. J. Essent. Oil Res., 2005, 17, 301-303.

[340]. Yang, Y.C.; Lee, H.S.; Lee, S.H.; Clark, J.M.; Ahn, Y.J. Ovicidal and adulticidal activities of cinnamomum zeylanicum bark essential oil compounds and related compounds against pediculus humanus capitis (anoplura: pediculicidae). Int. J. Parasitol., 2005, 35, 1595-1600.

[341]. Chao, L.K.; Hua, K.F.; Hsu, H.Y.; Cheng, S.S.; Liu, J.Y.; Chang, S.T. Study on the antiinflammatory activity of essential oil from leaves of Cinnamomum osmophloeum. J. Agric. Food Chem., 2005, 53(18), 7274-7278.

[342]. Cao, H.; Urban, J.F.; Jr, Anderson, R.A. Cinnamon polyphenol extract affects immune responses by regulating antiand proinflammatory and glucose transporter gene expression in mouse macrophages. J. Nutr., 2008, 138, 833-840.

[343]. Kwon, H.K.; Jeon, W.K.; Hwang, J.S.; Lee, C.G.; So, J.S.; Park, J.A.; Ko, B.S.; Im, S.H. Cinnamon extract suppresses tumor progression by modulating angiogenesis and the effector function of CD8+ T cells. Cancer Lett., 2009, 18, 174182.

[344]. Bandara, T.; Uluwaduge, I.; Jansz, E.R. Bioactivity of cinnamon with special emphasis on diabetes mellitus: a review. Int. J. Food Sci. Nutr., 2012, 63, 380-386.

[345]. Ranasinghe, P.; Jayawardana, R.; Galappaththy, P.; Constantine, G.R.; de Vas Gunawardana, N.; Katulanda, P. Efficacy and safety of 'true' cinnamon (cinnamomum zeylanicum) as a pharmaceutical agent in diabetes: a systematic review and meta-analysis. Diab. Med., 2012, 29, 1480-1492.

[346]. Hassan, S.A.; Barthwal, R.; Nair, M.S.; Haque, S.S. Aqueous bark extract of cinnamomum zeylanicum: a potential therapeutic agent for streptozotocin- induced type 1 diabetes mellitus (T1DM) rats. Trop. J. Pharm. Res., 2012, 11, 429-435.

[347]. Chericoni, S.; Prieto, J.A.; Iacopini, P.; Cioni, P.; Morelli, I. In vitro activity of the essential oil of cinnamomum zeylanicum and eugenol in peroxynitrite-induced oxidative processes. J. Agric. Food Chem., 2005, 53, 4762-4765.

[348]. Mathew, S.; Abraham, T.E. Studies on the antioxidant activities of cinnamon (cinnamomum verum) bark extracts, through various in vitro models. Food Chem., 2004, 94, 520-528.

[349]. Marshall, J.M. Aloe vera gel: What is the evidence? Pharma Jr., 1990, 24, 360-362.

[350]. Surjushe, A.; Vasani, R.; Saple, D.G. Aloe Vera: A short review. Indian J. Dermatol., 2008, 53(4), 163-166.

[351]. Grindly, D., Reynolds, T. The Alove vera phenomenon: a review of the properties and modern uses of the leaf parenchyma gel. J. Ethnopharmacol., 1986, 16, 117-51.

[352]. Jessica Houdret. Aloe vera In: Practical Herb Garden Anness publishing Ltd.Hermes House 88-89 Blackfriars Road, London SEI 8HA, 2003a, 104.

[353]. Hashemi, S.A.; Madani, S.A.; Abediankenari, S. The review on properties of Aloe Vera in healing of cutaneous wounds. BioMed Res. Int., 2015, 1-6.

[354]. West, D.P.; Zhu, Y.F. Evaluation of Aloe vera gel gloves in the treatment of dry skin associated with occupational exposure. Am. J. Infect. Contr., 2003, 31(1), 40-42.

[355]. Sahu, P.K.; Giri, D.D.; Singh, R.; Pandey, P.; Gupta ,S.; Shrivastava, A.K.; Kumar, A.; Pandey, K.D. Therapeutic and Medicinal Uses of Aloe vera: A Review. Pharmacol. Pharmacy, 2013, 4, 599-610.

[356]. Chithra, P.; Sajithlal, G.; Chandrakasan, G. Influence of aloe vera on the glycosaminoglycans in the matrix of healing dermal wounds in rats. J. Ethnopharmacol., 1998, 59, 179-86.

[357]. Moon, E.J.; Lee, Y.M.; Lee, O.H. A novel angiogenic factor derived from Aloe vera gel: $\beta$-sitosterol, a plant sterol. Angiogenesis, 1999, 3(2): 117-123.

[358]. Cardenas, C.; Quesada, A.R.; Medina, M.A. Evaluation of the anti-angiogenic effect of aloe-emodin. Cell Mol. Life Sci., 2006, 63(24), 3083-3089.

[359]. Rosca-Casian, O.; Parvu, M.; Vlase, L.; Tamas, M. Antifungal activity of Aloe vera leaves. Fitoterpia, 2007, 78(3), 219-222.

[360]. Vazquez, B.; Avila, G.; Segura, D.; Escalante, B. Anti-inflammatory activity of extracts from Alove vera gel. J. Ethnopharmacol., 1996, 55, 69-75.

[361]. Reddy, C.H.; Reddy, S.K.; Reddy, J. Aloe vera-A Wound Healer. Asian J. Oral Health Allied Sci., 2011, 1, 91-92.

[362]. Stuart, R.W.; Lfkowitz, D.L.; Lincoln, J.A.; Howard, K.; Gelderman, M.P.; Lefkowitz, S.S. Up-regulation of phagocytosis and candidicidal activity of macrophages exposed to immunostimulant acemannan. Int. J. Immunopharmcol., 1997, 19, 75-82.

[363]. Park, M.Y.; Kwon, H.J.; Sung, M.K. Evaluation of aloin and aloe-emodin as anti-inflammatory agents in aloe by using murine macrophages. Biosci. Biotechnol. Biochem., 2009, 73(4), 828-832.

[364]. Im, S.A.; Lee, Y.R.; Lee, Y.H.; Lee, M.K.; Park, Y.I.; Lee, S.; Kim, K.; Lee, C.K. In vivo evidence of the immunomodulatory activity of orally administered Alove vera gel. Arch. Pharm. Res., 2010, 33(3), 451-456.

[365]. Stengler, M. Aloe vera In: The natural physician's healing therapies. Bottomline books edition. 2005a, 18- 21.

[366]. Arora, S.; Kaur, K.; Kaur, S. Indian medicinal plants as a reservoir of protective phytochemicals. Teratog. Carcinog. Mutagen., 2003, (suppl.1), 295-300.

[367]. Kim, H.J.; Yokozawa, T.; Kim, H.Y.; Tohda, C.; Rao, T.P.; Juneja, L.R. Influence of amla (Emblica officinalis Gaertn.) on hypercholesterolemia and lipid peroxidation in cholesterol-fed rats. J. Nutr. Sci. Vitaminol. Tokyo., 2005, 51, 413-418.

[368]. Singh, E.; Sharma, S.; Pareek, A.; Dwivedi, J.; Yadav, S.; Sharma, S. Phytochemistry, traditional uses and cancer chemopreventive activity of Amla (Phyllanthus emblica): The Sustainer. J. Appl. Pharm. Sci., 2011, 02(01), 176-183. 
[369]. Jain, S.K. Ethnobotany and research on medicinal plants in India. Ciba Found. Symp., 1994,185, 153-164.

[370]. Ihantola-Vormisto, A.; Summanen, J.; Kankaaranta, H.; Vuorela, H.; Asmawi, Z.M.; Moilanen, E. Anti-inflammatory activity of extracts from leaves of Phyllanthus emblica. Plant. Med., 1997, 63, 518-524.

[371]. Khan, K.H. Roles of Emblica officinalis in Medicine - A Review. Botany Res. Int., 2009, 2(4), 218-228.

[372]. Singh, M.K.; Dwivedi, S.; Yadav, S.S.; Sharma, P.; Khattri, S. arsenic-induced hepatic toxicity and its attenuation by fruit extract of Emblica officinalis (Amla) in Mice. Indian J. Clin. Biochem., 2014, 29(1), 29-37.

[373]. Patel, A.P.; Bhagwat, S.R.; Pawar, M.M.; Prajapati, K.B.; Chauhan, H.D.; Makwana, R.B. Evaluation of Emblica officinalis fruit powder as a growth promoter in commercial broiler chickens, Vet. World, 2016, 9(2), 207-210.

[374]. Muhammad, S.A.; Ayesha, R.; Amanat, A.; Ahmad, M. Effect of amla fruit (Emblica officinalis Gaertn.) on blood glucose and lipid profile of normal subjects and type 2 diabetic patients. Int. J. Food Sci. Nutr., 2011, 62, 606-616.

[375]. Freedman, J.E. Oxidative stress and platelets. Arterioscler. Thromb. Vasc. Biol., 2008, 28, s11-s16.

[376]. Fatima, N.; Pingali, U.; Muralidhar, N. Study of pharmacodynamic interaction of Phyllanthus emblica extract with clopidogrel and ecosprin in patients with type II diabetes mellitus. Phytomed., 2014, 21(5), 579-585.

[377]. Jaijoy, K.; Soonthornchareonnon, N.; Panthong, A.; Sireeratawong, S. Anti-inflammatory and analgesic activities of the water extract from the fruit of Phyllanthus emblica Linn. Int. J. Appl. Res. Nat. Prod., 2010, 3(2), 28-35.

[378]. Saeed, S.; Tariq, P. Antibacterial activities of Emblica officinalis and Coriandrum sativum against Gram negative urinary pathogens. Pak. J. Pharm. Sci., 2007, 20(1), 32-5.

[379]. Srikumar, R.; Parthasarathy, N.J.; Shankar, E.M.; Manikandan, S.; Vijayakumar, R.; Thangaraj, R.; Vijayananth, K.; Sheela, D.R.; Rao, U.A. Evaluation of the growth inhibitory activities of Triphala against common bacterial isolates from HIV infected patients. Phytother. Res., 2007, 21(5), 476-80.

[380]. Saini, A.; Sharma, S.; Chhibber, S. Protective efficacy of Emblica officinalis against Klebsiella pneumoniae induced pneumonia in mice. Indian J. Med. Res., 2008, 128(2), 188-189.

[381]. Suresh, K.; Vasudevan, D.M. Augmentation of murine natural killer cell and antibody dependent cellular cytotoxicity activities by Phyllanthus emblica, a new immunomodulator. J. Ethnopharmacol., 1994, 44, 55-60.

[382]. Ganju, L.; Karan, D.; Chanda, S.; Srivastava, K.K.; Sawhney, R.C.; Selvamurthy, W. Immunomodulatory effects of agents of plant origin. Biomed Pharmacother., 2003, 57(7), 296-300.

[383]. Saravanan, S.R.; Srikumar, S.; Manikandan, N.J.; Parthasarathy, and Sheela, D.R. Hypolipidemic effect of triphala in experimentally induced hypercholesteremic rats. Yakugaku Zasshi., 2007, 127(2), 385-388.

[384]. Paul, R.; Khanna, A. A study of immunomodulatory effects of aqueous extract of Phyllanthus emblica (amla) leaf as a dietary herbal constituent in Clarias batrac hus by analyzing the haematological parameters. Inter. Res. J. Pharmacy., 2016, 7(1), 33-36.

[385]. Akhouri, S.; Prasad, A.; Ganguly, S. Moringa oleifera leaf extract imposes better feed utilization in broiler chicks. $J$. Biol. Chem. Res., 2013, 30(2), 447-450.

[386]. Akhouri, S.; Prasad, A.; Ganguly, S. Immunomodulatory effect of Moringa oleifera leaf extract in broiler chicks. Indian Vet. J., 2014a, $91(2), 52-4$.

[387]. Akhouri, S.; Prasad, A.; Ganguly, S. Experimental study of M. oleifera leaf extract in aqueous and powder formulations on immune response of chicks by Nitroblue Tetrazolium Reduction Test. Int. J. Chem. Pharma. Sci. 2014b, 2(5), 849-51.

[388]. Akhouri, S.; Prasad, A.; Ganguly, S. Overall effect of supplementation of M. oleifera leaf extract on hematological parameters of broiler chicks. Int. J. Pharm. Natural Medi. 2014c, 2(1), 102-106.

[389]. Berkovich, L.; Earon, G.; Ron, I.; Rimmon, A.; Vexler, A.; Lev-Ari, S. Moringa Oleifera aqueous leaf extract downregulates nuclear factor-kappaB and increases cytotoxic effect of chemotherapy in pancreatic cancer cells. $B M C$ Complement. Altern. Med., 2013, 13, 212. doi: 10.1186/1472-6882-13-212.

[390]. Gupta, A.; Gautam, M..;, Singh, R.K.; Kumar, M.V.; Rao, V.C.; Goel, R.K.; Anupuba, S. Immunomodulatory effect of M. oleifera in cyclophoshamide induces mice. Indian J. Exp. Biol., 2010, 48, 1157-60.

[391]. Bukar, A.; Uba, A.; Oyeyi, T.I. Antimicrobial profile of Moringa oleifera Lam. extracts against some food born microorganism. Bayero J. Pure Appl. Sci., 2010, 3(1), 43-48.

[392]. Saminathan, M.; Rai, R.B.; Dhama, K.; Tiwari, R.; Chakraborty, S.; Amarpal, Ranganath, G.J.; Kannan, K. Systematic review on anticancer potential and other health beneficial pharmacological activities of novel medicinal plant Morinda citrifolia (Noni). Int. J. Pharmacol., 2013, 9(8), 462-492.

[393]. Sunder, J.; Sujatha, T.; Kundu, A. Effect of Morinda citrifolia in growth, production and immunomodulatory properties in livestock and poultry: a review. J. Exper. Biol., 2016, 4, 3S: 249-265.

[394]. Kumar, S.K.; Suresh, M.; Kumar, S.A.; Kalaiselvi, P. Bioactive compounds, radical scavenging, antioxidant properties and FTIR spectroscopy study of Morinda citrifolia fruit extracts. Int. J. Curr. Microbiol. Appl. Sci., 2014, 3, $28-42$.

[395]. Wang, M.Y.; Peng, L.; Jensen, C.J.; Deng, S.; West, B.J. Noni juice reduces lipid peroxidation-derived DNA adducts in heavy smokers. Food sci. Nutri., 2013, 1(2), 141-149.

[396]. Almeida-Souza, F.; de Souza, C.D.S.F.; Taniwaki, N.N.; Silva, J.J.; M., de Oliveira, R.M.; Abreu-Silva, A.L.; da Silva Calabrese, K. Morinda citrifolia Linn. fruit (Noni) juice induces an increase in NO production and death of Leishmania amazonensis amastigotes in peritoneal macrophages from BALB/c. Nitric Oxide, 2016, 58, 51-58.

[397]. Coutinho de Sousa, B.; Reis Machado, J.; da Silva, M.V.; da Costa, T.A.; Lazo-Chica, J.E.; Degasperi, T.D.P.; Junior V.R.; Sales-Campos, H.; Bucek, E.U.; Freire Oliveira, C.J. Morinda citrifolia (Noni) fruit juice reduces inflammatory cytokines expression and contributes to the maintenance of intestinal mucosal integrity in DSS experimental colitis. Med,. Inflam., 2017.

[398]. Saraphanchotiwitthayaa, A.; Sripalakitb, P. Anti-inflammatory effect of Morinda citrifolia leaf extract on macrophage RAW 264.7 cells. Med., 2015, 7, 8 . 
[399]. Nayak, S.; Mengi, S. Immunostimulant activity of noni (Morinda citrifolia) on T and B lymphocytes. Pharm. biol., 2010, 48(7), 724-731.

[400]. Alsaeed, A.K.Z. Use of Morinda citrifolia (Noni). Int. J. Trop. Med., 2013, 8(4), 99-108.

[401]. Parikh, D.; Aggarwal, S.; Tandale, A.S.; Rai, V.; Borse, N.; Bhargava, K. Comparative evaluation of the antimicrobial activity of morinda citrifolia when used as an intracanal medicament with and without chitosan and propylene glycol on E. faecalis. World J. Pharm. Pharm. Sci., 2016, 1217-1225.

[402]. Sasmito, E.; Hertiani, T.; Kartika, S.; Putri, F.M.; Setiawan, V.; Narastika, L. Optimization of polysaccharide-rich fractionation from morinda citrifolia 1 . fruit based on immunostimulatory effect in vitro. Indonesian J. Pharmacy., 2015, 26(2), 78 .

[403]. Barani, K.; Manipal, S.; Prabu, D.; Ahmed, A.; Adusumilli, P.; Jeevika, C. Anti-fungal activity of Morinda citrifolia (noni) extracts against Candida albicans: An in vitro study. Indian J. Dental Res., 2014, 25(2), 188.

[404]. Ratnoglik, S.L.; Aoki, C.; Sudarmono, P.; Komoto, M.; Deng, L.; Shoji, I.; Fuchino, H.; Kawahara, N.; Hotta, H. Antiviral activity of extracts from Morinda citrifolia leaves and chlorophyll catabolites, pheophorbide a and pyropheophorbide a, against hepatitis C virus. Microbiol. Immunol., 2014, 58(3), 188-194.

[405]. Wang, J.; Qin, X.; Chen, Z.; Ju, Z.; He, W.; Tan, Y.; Zhoum X.; Tu, Z.; Lu, F.; Liu, Y. Two new anthraquinones with antiviral activities from the barks of Morinda citrifolia (Noni). Phytochem. Lett., 2016, 15, 13-15.

[406]. Mehrotra, P.; Rawat, S.; Kulshreshtha, D.K.; Patnaik, G.K.; Dhawan, B.N. Invitro studies on the effect of certain natural products against hepatitis B virus. Indian J. Med. Res., 1990, 92, 133-138.

[407]. Thyagarajan, SP.; Jayaram, S.; Gopalakrishnan, V.; Hari, R.; Jeyakumar, P.; Sripathi, M.S. Herbal medicines for liver diseases in India. J. Gastroent. Hepatol., 2008, 17(3), S370-S376.

[408]. Rajaprabhu, D.; Rajesh, R.; Jeyakumar, R.; Buddhan, S.; Ganesan, B.; Anandan, R. Protective effect of Picrorhiza kurroa on antioxidant defense status in adriamycin-induced cardiomyopathy in rats. J. Med. Plant Res., 2007, 1(4), $80-85$.

[409]. Rajkumar, V.; Gunjan Guha R.; Kumar, A. Antioxidant and anti-neoplastic activities of Picrorhiza kurroa extracts. Food Chem. Toxicol., 2011, 49(2), 363-369.

[410]. Baruah, C.C.; Gupta, P.P.; Nath, A.; Patnaik, G.K.; Dhawan, B.N. Anti-allergic and anti-anaphylactic activity of Picroliv - a standardized iridoid glycoside fraction of Picrorhiza kurroa. Pharmacol. Res., 1998; 38(6), 487-492.

[411]. Kumar, A.R.; Rajkumar, V.; Guha, G.; Mathew, L. Therapeutic potentials of Oroxylum indicum bark extracts. Chin. J. Nat. Med., 2010, 8(2), 0121-0126.

[412]. Sultan, P.; Jan, A.; Pervaiz, Q.; Phytochemical studies for quantitative estimation of iridoid glycosides in Picrorhiza kurroa Royle. Botanical Stud., 2016, 57, 7.

[413]. Shetty, S.N.; Mengi, S.; Vaidya, R.; Vaidya, A.D.B. A study of standardized extracts of Picrorhiza kurroa Royle ex Benth in experimental nonalcoholic fatty liver disease, J. Ayurveda. Integr. Med., 2010, 1(3), 203-210.

[414]. Sidiq, T.; Khajuria, A.; Suden, P.; Sharma, R.; Singh, S.; Suri, K.A.; Satti, N.K.; Johri, R.K. Possible role of macrophages induced by an irridoid glycoside (RLJ-NE-299A) in host defense mechanism. Int. Immunopharmacol., 2011, 11(1), 128-135.

[415]. Gupta, A.; Khajuria, A.; Singh, J.; Bedi, K.L.; Satti, N.K.; Dutt, P.; Suri, K.A.; Suri, O.P.; Qazi, G.N. Immunomodulatory activity of biopolymeric fraction RLJ-NE-205 from Picrorhiza kurroa. Int. Immunopharmacol., 2006, 6(10), 1543-1549.

[416]. Masood, M.; Arshad, M.; Qureshi, R.; Sabir, S.; Amjad, M.S.; Qureshi, H. and Tahir. Z. Picrorhiza kurroa: An ethnopharmacologically important plant species of Himalayan region. Pure Appl. Biol., 2015, 4(3), 407-417.

[417]. Puri, A.; Saxena, R.P.; Sumati, Guru, P.Y.; Kulshreshta, D.K.; Saxena, K.C.; Dhawan, B.N. Immunostimulant activity of picroliv, the iriod glycoside fraction of Pirorhiza kurroa and its protective action against Leishmania donavani infection in hamsters. Planta Med., 1992, 58, 528-532.

[418]. Hussain, A.; Shadma, W.; Maksood, A.; Ansari, S.H. Protective effects of Picrorhiza kurroa on cyclophosphamideinduced immunosuppression in mice. Phcog. Res., 2013, 5, 30-35.

[419]. Latto, S.K.; Khan, S.; Dhar, A.K.; Chaudhry, D.K.; Gupta, K.K.; Sharma, P.R. Genetics and mechanism of induced male sterility in Andrographis paniculata (Berm.f.) Nees and its significance. Curr. Sci., 2006, 91, 515-519.

[420]. Li, J.; Huang, W.; Zhang, H.; Wang, X.; Zhou, H. Synthesis of andrographolide derivatives and their TNF-alpha and IL-6 expression inhibitory activities. Bioorg. Med. Chem. Lett., 2007, 17, 6891-6894.

[421]. Mishra, S.K.; Sangwan, N.S.; Sangwan, R.S. Andrographis paniculata (Kalmegh): a review. Pharmacognosy Rev., 2007, 1, 283-298.

[422]. Jarukamjorn, K.; Kondo, S.; Chatuphonprasert, W.; Sakuma, T.; Kawasaki, Y.; Emito, N. Gender-associated modulation of inducible CYP1A1 expression by andrographolide in mouse liver. Eur. J. Pharm. Sci., 2010, 39, 394401.

[423]. Okhuarobo, A.; Falodun, J.E.; Erharuyi, O.; Imieje, V.; Falodun, A.; Langer, P. Harnessing the medicinal properties of Andrographis paniculata for diseases and beyond: a review of its phytochemistry and pharmacology. Asian Pac. J. Trop. Dis., 2014, 4(3), 213-222.

[424]. Singha, P.K.; Roy, S.; Dey, S. Antimicrobial activity of Andrographis paniculata. Fitoterapia, 2003, 74, $692-694$.

[425]. Woo, A.Y.; Waye, M.M.; Tsui, S.K. Andrographolide up-regulates cellular reduced glutathione level and protects cardiomyocytes against hypoxia/ reoxygenation injury. J. Pharmacol. Exp., 2008, 325, 226-235.

[426]. Akbar, S. Andrographis paniculata: A review of pharmacological activities and clinical effects. Altern. Med. Rev., 2011, 16(1), 66-77.

[427]. Chandrasekaran, C.V.; Gupta, A.; Agarwal, A. Effect of an extract of Andrographis paniculata leaves on inflammatory and allergic mediators in vitro. J. Ethnopharmacol., 2010, 129, 203-207. 
[428]. Lee, K.C.; Chang, H.H.; Chung,Y.H.; Lee,T.Y. Andrographolide acts as an anti-inflammatory agent in LPS-stimulated

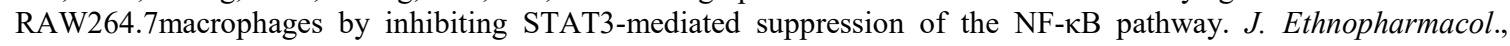
2011, 135, 678-684.

[429]. Zhang, Q.Q.; Ding, Y.; Lei,Y.; Qi, C.L.; He, X.D.; Lan, T. Andrographolide suppress tumor growth by inhibiting TLR4/NF-KB signalling activation in insulinoma. Int. J. Biol. Sci., 2014, 10, 404-414.

[430]. Jantan, I.; Ahmad, W.; Bukhari, S.N.A. Plant-derived immunomodulators: an insight on their preclinical evaluation and clinical trials. Front. Plant Sci., 2015, 6, 655

[431]. Burgos, R.A.; Hancke, J.L.; Bertoglio, J.C.; Aguirre, V.; Arriagada, S.; Calvo, M. Efficacy of an Andrographis paniculata composition for the relief of rheumatoid arthritis symptoms: a prospective randomized placebo- controlled trial. Clin. Rheumatol., 2009, 28, 931-946.

[432]. Tang, T.; Targan, S.R.; Li, Z.S.; Xu, C.; Byers, V.S.; Sandborn, W.J. Randomised clinical trial: herbal extract HMPL004 inactive ulcerativecolitis-a double-blind comparison with sustained release mesalazine. Aliment. Pharmacol. Ther., 2011, 33, 194-202.

[433]. Sandborn, W. J.; Targan,S.R.; Byers,V.S.; Rutty,D.A.; Mu,H.; Zhang, X. Andrographis paniculata extract (HMPL004) for active ulcerative colitis. Am. J.Gastroenterol., 2013, 108, 90-98.

[434]. Yadav, R.A.; Yadav, N.; Kharya, M.D. Immunomodulation potential of Andrographis paniculata and Tinospora cordifolia methanolic extracts in combination forms. Inter. J. Pharmacol. Res., 2016, 6(1), 29- 40.

[435]. Oketch-Rabah, HA. Phytochemical constituents of the Genus Asparagus and their biological activities. Hamdard, 1998, 41, 33-43.

[436]. Bopana, N.; Saxena, S. Asparagus racemosus-Ethnopharmacological evaluation and conservation needs. J. Ethnopharmacol., 2007, 110(1), 1-15.

[437]. Goyal, R.K.; Singh, J.; Lal, H. Asparagus racemosus--an update. Indian J. Med. Sci., 2003, 57, 408.

[438]. Joshi, J.; Dev, S. Chemistry of Ayurvedic crude drugs: Part VIIIa-Shatavari-2: Structure elucidation of bioactive Shatavarin-I \& other glycosidesb,c. Indian J. Chem., 1988, 27B, 12-16.

[439]. Pise, M.; Rudra, J.; Begde, D.; Nashikkar, N.; Upadhyay, A. Shatavarins production from in vitro cultures of Asparagus racemosus. J. Med. Plant Res., 2011, 5, 507-13.

[440]. Pise, M.; Rudra, J.; Upadhyay, A. Immunomodulatory potential of shatavarins produced from Asparagus racemosus tissue cultures. J. Nat. Sci. Biol. Med., 2015, 6(2), 415-420.

[441]. Parihar, M.S.; Hemnani, T. Experimental excitotoxicity provokes oxidative damage in mice brain and attenuation by extract of Asparagus racemosus. J. Neural Transm., 2004, 111, 1-12.

[442]. Kamat, J.P.; Boloor, K.K.; Devasagayam, T.P.A.; Venkatachalam, S.R. Antioxidant properties of Asparagus racemosus against damage induced by $\gamma$-radiation in rat liver mitochondria. J. Ethnopharmacol., 2000, 71, 425-435.

[443]. Muruganadan, S.; Garg, H.; Lal, J.; Chandra, S.; Kumar, D. Studies on the immunostimulant and antihepatotoxic activities of Asparagus racemosus root extract. J. Med. Arom. PI. Sci., 2000, 22, 49-52.

[444]. Seena, K.; Kuttan, G.; Kuttan, R. Antitumor activity of selected plant extracts. Amla Res Bull., 1993, 13, 41-45.

[445]. Satti, N.K.; Suri, K.A.; Dutt, P.; Suri, O.P.; Musrat, A.; Qazi, G.N. Evaluation of Asparagus racemosus on the basis of immunomodulating sarasapogenin glycosides by HPTLC. J Liq Chromatogr Relat Technol., 2006, 29,119-227.

[446]. Gautam, M.; Saha, S.; Bani, S.; Kaul, A.; Mishra, S.; Patil, D.; Satti, K.; Suri, K.A.; Gairola, S.; Suresh, K.; Jadhav, S.; Qazib, G.N.; Patwardhan, B. Immunomodulatory activity of Asparagus racemosus on systemic Th1/Th2 immunity: Implications for immunoadjuvant potential. J. Ethnopharmacol., 2009, 121(2),241-247.

[447]. Thatte, U.M.; Dahanukar, S.A. Comparative study of immunomodulating activity of Indian medicinal plants, Lithium carbonate and Glucan; Methods. Find Expt. Clin. Pharmacol., 1988, 10, 639-644.

[448]. Lee, D.Y.; Choo, B.K.; Yoon, T. Anti-inflammatory effects of Asparagus cochinchinensis extract in acute and chronic cutaneous inflammation. J. Ethnopharmacol., 2009, 121(1), 28-34.

[449]. Sharma, P.; Chauhan, P.S.; Dutt, P.; Amina, M.; Suri, K.A.; Gupta, B.D.; Suri, O.P.; Dhar, K.L.; Sharma, D.; Gupta, V.; Satti, N.K. A unique immuno-stimulant steroidal sapogenin acid from the roots of Asparagus racemosus. Steroids, 2011, 76(4), 358-64.

[450]. Alok, S.; Jain, S.K.;Verma, A.; Kumar, M.; Mahor, A.; Sabharwal, M. Plant profile, phytochemistry and pharmacology of Asparagus racemosus (Shatavari): A review. Asian Pac. J. Trop. Dis., 2013, 3(3), $242-251$.

[451]. Bhargava, K.P.; Singh, N. Anti-stress activity in Indian medicinal plants. Res Educ bid Med., 1985, 27, 3-4.

[452]. Dua, P.R.; Shankar, G.; Srimal, R.C.; Saxena, K.C.; Saxena, R.P.; Puri, A.; Dhawan, B.N. Adaptogenic activity of Indian Panax pseudoginseng. Indian J. Exp. Biol., 1989, 27, 631-634.

[453]. Gao, H.; Wang, F.; Lien, E.J.; Trousdale, M.D. Immunostimulating polysaccharide from Panax notoginseng. Pharm. Res., 1996, 13, 1196-1200.

[454]. Castleman, M. Ginseng. In: the new healing herbs Third Ed. Rondale Inc.USA, 2009d, 250-258.

[455]. Grandhi, A.; Mujumdar, A.M.; Patwardhan, B. A comparative pharmacological investigation of Ashwagandha and Ginseng. J. Ethnopharmacol., 1994, 44, 131-135.

[456]. Tran, Q.L.; Adnyana, K.; Tezuka, Y.; Nagaoka, T.; Tran, Q.K.; Kadota, S. Triterpene Saponins from Vietnamese Ginseng (Panax vietnamensis) and Their Hepatocytoprotective Activity. J. Nat. Prod., 2001, 64(4), 456-461.

[457]. Xiaoguang, C.; Hongyan, L.; Xiaoguang, L.; Zhoadi, F.; Van, L.; Lihua, T.; Rui, H. Cancer chemopreventive and therapeutic activities of red ginseng. J. Ethnopharmacol., 1998, 60, 71-78.

[458]. Zee-Cheng, R.K. Shi-quan-da-bu-tang (ten significant tonic decoction), SQT. A potent Chinese biological response modifier in cancer immunotherapy, potentiation and detoxification of anticancer drugs. Methods Find Exp. Clin. Pharmacol., 1992, 14, 725-736. 
[459]. Tomoda, M.; Takeda, K.; Shimizu, N.; Gonda, R.; Ohara, N.; Takeda, K.; Hirabayashi, K. Characterization of two acidic polysaccharides having immunological activities from the root of Panax ginseng. Biol. Phann. Bull., 1993, 16, $22-25$.

[460]. Mizuno, M.; Yamada, J.; Teral, H.; Kozukue, N.; Lee, Y.S., Tsuchida, H. Differences in immunomodulating effects between wild and cultured Panax ginseng. Biochem. Biophys. Res. Commun., 1994, 16, 1672-1678.

[461]. Solo'veva, T.F.; Besednova, N.N.; Uvarova, N.I.; Faustov, V.S.; Konstantinova, N.A.; Krylova, N.V.; Tsybulskii, A.V.; Ovodov, IuS.; Eliakov, G.B. Phagocytosis-stimulating effect of polysaccharides isolated from ginseng tissue culture. Antibiot Khimioter, 1989, 34, 755-760.

[462]. Scaglione, F.; Ferrara, F.; Dugnani, S.; Falchi, M.; Santoro, G.; Fraschini, F. Immunomodulatory effects of two extracts of Panax ginseng C. A. Meyer; Drugs. Exp Clin Res., 1990, 16, 537-542.

[463]. Lee, Y.S.; Chung, I.S.; Lee, I.R.; Kim, K.H.; Hong, W.S.; Yum, Y.S. Activation of multiple effector pathways of immune system by the antineoplastic immunostimulator acidic polysaccharide ginsan isolated from Panax ginseng. Anticancer Res., 1997, 17, 323-331.

[464]. See, D.M.; Broumand, N.; Sahl, L.; Tilles, J.G. In vitro effects of echinacea and ginseng on natural killer and antibody dependent cell cytotoxicity in healthy subjects and chronic fatigue syndrome or acquired immunodeficiency syndrome patients. Immunopharmacol., 1997, 35, 229-235.

[465]. Kim, K.H.; Lee, V.S.; Jung, I.S.; Park, S.Y.; Chung, H.Y.; Lee, I.R.; Vun, Y.S. Acidic polysaccharide from Panax ginseng, ginsan, induces Th1 cells and macrophage cytokines and generates LAK cells in synergy with rIL-2. Planta Med., 1998, 64, 110-115.

[466]. Kenarova, B.; Neychev, H.; Hadjiivanova, C.; Petkov, V.D. Immunomodulating activity of ginsenoside Rgl from Panax ginseng. Jpn. J. Pharmacol., 1990, 54, 447-454.

[467]. Nikitina, Z.K.; Ludaeva, F.A.: Aleksandrova, I.V.The role of proteins in the immunomodulating effect of bioginsing products. Vopr. Med.Khim., 1995, 41, 30-32.

[468]. Ma, L.1.; Zhou, Z.L.; Yang, Q. Study on effect of polysaccharides of ginseng on peripheral blood mononuclear cell induced interleukin-2 production and activity of its receptors in vitro. Zhongguo Zhong Xi Yi Jie He Za Zhi.1995, 15(7), 411-413.

[469]. Yeung, H.W.; Cheung, K.; Leung, K.N. Immunopharmacology of Chinese medicine, ginseng induced immunosuppression in virus infected mice. Am. J. Chin. Med., 1982, 10, 44-54.

[470]. Lee, J.S.; Lee, Y.; Lee, Y.; Hwang, H.S.; Kim, K.; Ko, E.; Kim, M.; Kang, S. Ginseng protects against respiratory syncytial virus by modulating multiple immune cells and inhibiting viral replication. Nutrients, 2015, 7(2), 1021-1036.

[471]. Quan, F.S.; Compans, R.W.; Cho, Y.K.; Kang, S.M. Ginseng and Salviae herbs play a role as immune activators and modulate immune responses during influenza virus infection. Vaccine, 2007, 25, 272-82.

[472]. Sakure, S.; Negi, V.D.; Mitra, S.K.; Nandakumar, K.S.; Chakravortty, D. Vaccine with herbal adjuvant-A better cocktail to combat the infection. Vaccine, 2008, 26(2008), 3387-3388.

[473]. Kim, H.; Jang, M.; Kim, Y.; Choi, J.; Jeon, J.; Kim, J.; Hwang, Y.I.; Kang, J.S.; Lee, W.J. Red ginseng and vitamin C increase immune cell activity and decrease lung inflammation induced by influenza A virus/H1N1 infection. J. Pharm. Pharmacol., 2016, 68(3), 406-420. doi: 10.1111/jphp.12529. Epub 2016 Feb 21.

[474]. Yang, L.; Liu, Y.; Liu, C.X. Metabolism and pharmacokinetics of ginsenosides. Asian J. Pharmacodyn. Pharmacokin., 2006, 6(2), 103-120.

[475]. Tawab, M.A.; Bahr, U.; Karas, M.; Wurglics, M.; Schubert-Zsilavecz, M. Degradation of ginsenosides in humans after oral administration. Drug Metab. Dispos., 2003, 31, 1065-1071.

[476]. Bao, P.P.; Lu, W.; Cui, Y.; Xheng, Y.; Gu, K.; Chen, Z.; Xheng, W.; Shu, X.O. Ginseng and Ganoderma lucidum use after breast cancer diagnosis and quality of life: a report from the Shanghai Breast Cancer Survival Study. PLoS One, 2012.7(6), e39343.

[477]. Wu, G.S.; Lu, J.J.; Guo J.J.; Li, Y.B.; Tan, W.; Dang, Y.Y.; Zhong, Z.F.; Xu, Z.T.; Chen, X.P.; Wang, Y.T. Ganoderic acid DM, a natural triterpenoid, induces DNA damage, G1 cell cycle arrest and apoptosis in human breast cancer cells. Fitoterapia, 2012, 83(2), 408-414

[478]. Xu, Z.; Chen, X.; Zhong, Z.; Chen, L.; Wang, Y. Ganoderma lucidum polysaccharides: immunomodulation and potential anti-tumor activities. Am. J. Chinese Med., 2011, 39(1), 15-27.

[479]. Liao, G.S.; Apaya, M.K.; Shyur, L.F. Herbal medicine and acupuncture for breast cancer palliative care and adjuvant therapy. Evid. Based Complement. Alter. Med., 2013, e437948.

[480]. Aeschbach, R.; Loliger, J.; Scott, B.C.; Muscia, A.; Butler, J.; Halliwell, B. Antioxidant action of thymol, carvacrol, 6ginerol, zinezerone and hydroxytyrosol. Food Chem. Toxicol., 1994, 32, 31-36.

[481]. Abd El-Hack, ME.; Alagawany, M.; Farag, M.R.; Tiwari, R.; Karthik, K.; Dhama, K.; Zorriehzahra, J.; Adel, M.; Beneficial impacts of thymol essential oil on health and production of animals, fish and poultry: a review. J. Essential Oil Res., 2016b, 28(5), 365-382.

[482]. Vincent, H.V. Carvacrol and thymol reduce swine waste odour and pathogens stability of oils. Curr. Microbiol., 2002, 44, 38-43.

[483]. Basilico, M.Z.; Basilico, J.C.; Inhibitory effects of some spice essential oils on Aspergillus ochraceus NRRL 3174 growth and ochratoxin A production. Lett. Appl. Microbiol., 1999, 29, 238-241.

[484]. Javed, H.; Erom, S.; Tabassum, S.; Ameen, S. An overview on medicinal importance of Thymus vulgaris. J. Asian Scient. Res., 2013, 3(10), 974-982.

[485]. Twetman, S.; Peterson, L.G. Effect of different chlorhexidine varnish regimens on mutant streptococci levels in interdental plaque and saliva. Caries Res., 1997, 31, 189-193.

[486]. Allen, P.C.; Danforth, H.D.; Augustine, P.C. Diet modulation of avian coccidiosis. Int. J. Parasitol., 1998, 28, 11311140. 
[487]. Denli, M.; Okan, F.; Uluocak, A.N. Effect of dietary supplementation of herb essential oils on the growth performance, carcass and intestinal characteristics of quail. South Africa. J. Anim. Sci., 2004, 34(3), 174-179.

[488]. Cross, D.E.; McDevitt, R.M.; Hillman, K.; Acamovic, T. The effect of herbs and their associated essential oils on performance, dietary digestibility and gut microflora in chickens from 7 to 28 days of age. Br. Poult. Sci., 2007, 48(4), 496-506.

[489]. Abd El-Hack, M.E.; Alagawany, M. Performance, egg quality, blood profile, immune function, and antioxidant enzyme activities in laying hens fed diets with thyme powder. J. Anim. Feed Sci., 2015, 24(2), 127-133.

[490]. Karimi, A. The impact of adding the mixture of medicinal herbs to the diet on the qualitative characteristics of egg. Int. J. Anim. Vet. Adv., 2014, 6, 34-39.

[491]. Hashemipour, H.; Kermanshahi, H.; Golian, A.; Veldkamp, T.; Effect of thymol and carvacrol feed supplementation on performance, antioxidant enzyme activities, fatty acid composition, digestive enzyme activities, and immune response in broiler chickens. Poultry Sci., 2013, 92, 2059-2069.

[492]. Bharini A, Ganguly A, Bhargave KD. 1995. Salutary effect of Terminalia arjuna in patients with severe refractory heart failure. Int. Cardiol., 1985, 49, 191-199.

[493]. Subramaniam, S.; Ramachandran, S.; Uthrapathi, S.; Gnamanickam, V.R.; Dubey, G.P. Anti-hyperlipidemic and antioxidant potential of different fractions of Terminalia arjuna Roxb. bark against PX-407 induced hyperlipidemia. Indian J. Exp. Biol., 2011, 49(4), 282-288.

[494]. Bone, K. Clinical applications of Ayurvedic and Chinese herbs. Warwick, Queensland, Australia. Phytotherapy Press, 1996, 131-133.

[495]. Ansari, J.A.; Neeraj, Singh, A.; Sushma, R.P. Effects of dietary Arjuna (Terminalia arjuna) Bark powder supplementation on serum biochemical of broilers. Indo Am. J. Pharm. Sci., 2016, 3(1), 1-4.

[496]. Ganesan, R.; Kamalraj, P.; Muthuchelian, K. Protective effects of ethanolic extract residue isolated from the bark of Terminalia arjuna against DLA tumour cells. Asian Pac. J. Cancer Prev., 2010, 11(3), 803-808.

[497]. Sahil, T.; Nandakumar, K.; Pawan, G.N.; Bansal, P.; Jayesh, M.; Mor, V.; Mallikarjuna, R.C.; Richard, L. Antiinflammatory activity of Terminalia paniculata bark extract against acute and chronic inflammation in rats. $J$. Ethnopharmacol., 2010, 134(2), 323-328.

[498]. Hemalatha, T.; Pulavendran, S.; Balachandran, C.; Manohar, B.M.; Puvanakrishnan, R. Arjunolic acid: a novel phytomedicine with multifunctional therapeutic applications. Indian J. Exp. Biol., 2010, 48, 238-247.

[499]. Kapoor, D.; Trikha, D.; Vijayvergiya, R.; Parashar, K.K.; Kaul, D.; Dhawan, V. Short-term adjuvant therapy with terminalia arjuna attenuates ongoing inflammation and immune imbalance in patients with stable coronary artery disease: in vitro and in vivo evidence. J. Cardiovasc. Transl. Res., 2015, 8(3), 173-86.

[500]. Hussain, A.; Khan, M.N.; Sajid, M.S.; Iqbal, Z.; Khan, M.K.; Abbas, R.Z.; Raza, M.A.; Needham, G.R. In vitro screening of Ziziphus mauritiana and Terminalia arjuna for their anthelmintic activity. J. Anim. Plant Sci., 2011, 20, $5-8$.

[501]. Mandal, S.; Patra, A.; Samanta, A.; Roy, S.; Mandal, A.; Mahapatra, T.D.; Pradhan, S.; Das, K.; Nandi, D.K. Analysis of phytochemical profile of Terminalia arjuna bark extract with antioxidative and antimicrobial properties. Asian Pac. J. Trop. Biomed., 2013, 3(12), 960-966.

[502]. Varghese, A.; Savai, J.; Pandita, N.; Gaud, R. In vitro modulatory effects of Terminalia arjuna, arjunic acid, arjunetin and arjungenin on CYP3A4, CYP2D6 and CYP2C9 enzyme activity in human liver microsomes. Toxicol. Reports, 2015, 2(2015), 806-816.

[503]. Amalraj, A.; Gopi, S. Medicinal properties of Terminalia arjuna (Roxb.) Wight \& Arn.: A review. J. Trad. Complement. Med., 2017, 65e78.

[504]. Wu, X.; Cao, G.; Prior, R.L. Absorption and metabolism of anthocyanins in elderly women after consumption of elderberry or blueberry. J. Nutr., 2002, 132, 1865-1871.

[505]. Milbury, P.E.1; Cao, G.; Prior, R.L.; Blumberg, J. Bioavailablility of elderberry anthocyanins. 2002, 123(8), 9971006.

[506]. Ulbricht, C.; Goldgerb, H. An evidence-based systematic review of Elderberry and Elderflower (Sambucus nigra) by the natural standard research collaboration. J. Diet. Suppl., 2014, 11(1), 80-120.

[507]. Sahpira-Nahor , O.; Zakay-Rones , Z.; Mumcuoglu, M. The effects of Sambucol® on HIV infection in vitro. Ann. Israel Congress Microbiol., 1995, 6-7.

[508]. Morag, A.M.; Mumcuoglu, M.; Baybikov, T. Inhibition of sensitive and acyclovir-resistant HSV-1 strains by an elderberry extract in vitro. Phytother., 1997, 25, 97-98.

[509]. Zakay-Rones, Z.; Thom, E.; Wollan, T.; Wadstein, J. Randomized study of the efficacy and safety of oral elderberry extract in the treatment of influenza A and B virus infections. J. Int. Med. Res., 2004, 32, 132-140.

[510]. Janeway, C.A. Jr.; Travers, P.; Walport, M.; Shlomchik, M.J. The Immune System in Health and Disease. New York, NY: Garland Publishing, 2001, 12-13.

[511]. Badescu, M.; Badulescu, O.; Badescu, L.; Ciocoiu, M. Effects of Sambucus nigra and Aronia melanocarpa extracts on immune system disorders within diabetes mellitus. Pharm. Biol., 2015, 53(4), 533-539. doi: 10.3109/13880209.2014.931441.

[512]. Kirtikar, K.R.; Basu, B. Indian Medicinal Plants. International Book Publisher, 1993, 2, 898- 900.

[513]. Nadkarni, AK. Indian Materia Medica. New Delhi, Popular Directorate, CSIR, 2001, 1, 56.

[514]. Arvind, N.; Sharma, N.; Singh, M.F. Spectrum of pharmacological activities from Bauhinia variegata: A review. J. Pharm. Res., 2012, 5(2), 792-797.

[515]. Parekh, J.; Karathia, N.; Chandra, S. Evaluation of antibacterial activity and phytochemical analysis of Bauhinia variegata $L$ bark. Afr. J. Biomed. Res., 2006, 9, 53-56. 
[516]. Dewanagan, P.; Verma, A.; Kesharwani, D. Isolation of D- Pinitol: A bioactive carbohydrate from the leaves of Bauhinia variegata L. Int. J. Pharm. Sci. Rev. Res., 2014, 24(1), 43-45.

[517]. Sharma, K.R.; Kalauni, S.K; Awale, S. Antioxidant, phytotoxic and antimicrobial activities of methanolic extract of Bauhinia variegata barks. J. Inst. Sci. Tech., 2015, 20(2), 37-41.

[518]. Mishra, A.; Sharma, A.K.; Kumar, S.; Saxena, A.K.; Pandey , A.K. Bauhinia variegata leaf extracts exhibit considerable antibacterial, antioxidant, and anticancer activities. Biomed Res. Int., 2013, e915436.

[519]. Al-Snafi, A.E. The pharmacological importance of Bauhinia variegata. A review. Int. J. Pharm. Sc. Res., 2013, 4(12): 160-164.

[520]. Boon, H.; Smith, M. The Botanical Pharmacy. Kingston, Ontario: Quarry Press, 1999, 194.

[521]. Huang, L.; Zhao, H.; Huang, B.; Zheng, C.; Peng, W.; Qin, L. Acanthopanax senticosus: review of botany, chemistry and pharmacology. Pharmazie, 2011, 66(2), 83-97.

[522]. Deyama, T.; Nishibe, S.; Nakazawa, Y. Constituents and pharmacological effects of Eucommia and Siberian ginseng. Acta Pharmacol. Sin., 2001, 22, 1057-107.

[523]. Mills, S.; Bone, K. Principles and Practice of Phytotherapy. New York, NY, Churchill Livingston, $2000,536$.

[524]. Nishibe, S.; Kinoshita, H.; Takeda, H.; Okano, G. Phenolic compounds from stem bark of Acanthopanax senticosus and their pharmacological effect in chronic swimming stressed rats. Chem. Pharm. Bull. (Tokyo)., 1990, 38, 17631765.

[525]. Tang, W.; Eisenbrand, G. Chinese Drugs of Plant Origin. Heidelberg, Germany: Springer Verlag, 1992.

[526]. Steinmann, G.G.; Esperester, A.; Joller, P. Immunopharmacological in vitro effects of Eleutherococcus senticosus extracts. Arzneimittelforschung, 2001, 51, 76-83.

[527]. Bu, Y.; Jin, Z.H.; Park, S.Y.; et al. Siberian ginseng reduces infarct volume in transient focal cerebral ischaemia in Sprague-Dawley rats. Phytother. Res., 2005, 19, 167-169.

[528]. Park, E.J.; Nan, J.X.; Zhao, Y.Z. Water-soluble polysaccharide from Eleutherococcus senticosus stems attenuates fulminant hepatic failure induced by D-galactosamine and lipopolysaccharide in mice. Basic Clin. Pharmacol. Toxicol., 2004, 94, 298-304.

[529]. Kropotov, A.V.; Kolodnyak, O.L.; Koldaev, V.M. Effects of Siberian ginseng extract and ipriflavone on the development of glucocorticoid-induced osteoporosis. Bull. Exp. Biol. Med., 2002, 133, 252-254.

[530]. Kao, E.S.; Yang, M.; Hung, C.; Huang, C.; Wang, C. Polyphenolic extract from Hibiscus sabdariffa reduces body fat by inhibiting hepatic lipogenesis and preadipocyte adipogenesis. Food Funct., 2016, 7, 171-182.

[531]. Rapavi, E.; González-Cabello, R.; Szentmihályi, K.; Székely, E.; Blázovics, A. The effect of calyx infusion of hibiscus sabdariffa on T-cells-mediated immune response in mitogen-induced blastogenesis of human lymphocytes in vitro. Acta Alimentaria, 2006, 35, 281-288.

[532]. Ologundudu, A.; Ologundudu, A.O.; Ololade, I.A.; Obi, O.F. Effect of Hibiscus sabdariffa anthocyanins on 2, 4dinitrophenylhydrazine-induced hematotoxicity in rabbits. Afr. J. Biochem. Res., 2009, 3(4), 140-144.

[533]. Beltran-Debon, R.; Alonso-Villaverde, C.; Aragonès, G.; Rodríguez-Medina, I.; Rull, A.; Micol, V.; Segura-Carretero, A.; Fernández-Gutiérrez, A.; Camps, J.; Joven, J. The aqueous extract of Hibiscus sabdariffa calices modulates the production of monocyte chemoattractant protein-1 in humans. Phytomed., 2010, 17(3-4), 186-189.

[534]. Farajia, M.H.; Tarkhanib, A.H. The effect of sour tea (Hibiscus sabdariffa) on essential hypertension. J. Ethnopharmacol., 1999, 65(3): 231-236.

[535]. Patel, V.R.; Patel, P.R.; Kajal, S.S. Antioxidant activity of some selected medicinal plants in western region of India. Adv. Biol. Res., 2010, 4(1), 23-26.

[536]. Goda, Y.; Hoshino, K.; Akiyama, H.; Ishikawa, T.; Abe, Y.; Nakamura, T.; Otsuka, H.; Takeda, Y.; Tanimura, A.; Toyoda, M. Constituents in Watercress, Inhibitors of Histamine Release from RBL-2H3 Cells Induced by Antigen Stimulation. Biol. Pharm. Bull., 1999, 22, 1319-1326.

[537]. Shahrokhi, N.; Hadad, M.K.; Keshavarzi, Z; Shabani, M. Effect of aqueous extract of watercress on glucose and lipid plasma in streptozotocin induced diabetic rats. Pak. J. Physiol., 2009, 5(2), 6-10.

[538]. Sonnenbichler, J.; Goldberg, M.; Hane, L.; Madubunyi, I.; Vogl, S.; Zetl, I. Stimulatory effect of silibinin on DNA synthesis in partially hepatectomized rat livers, non-response in hepatoma and other malign cell lines. Biochem. Pharmacol., 1986, 35, 538-541.

[539]. Yazdanparast, R.; Bahramikia, S.; Ardestani, A. Nasturtium officinale reduces oxidative stress and enhances antioxidant capacity in hypercholesterolemia rats. Chem. Biol. Interact., 2008, 172, 176-184.

[540]. Abu-Zinadah, O. Effects of Watercress oil on the thermal and chemical burn injuries in rabbits. JKAU, Med. Sci., 2008, 15(4), 3-17.

[541]. Chung, F.L.; Morse, M.A.; Eklind, K.I.; Lewis, J. Quantitation of human uptake of the anticarcinogen phenethyl isothiocyanate after a watercress meal. Cancer Epidemiol. Biomarkers Prev. 1992, 1(5), 383-388.

[542]. Palaniswamy, U.R.; McAvoy, R.J.; Bible, B.B.; Stuart, J.D. Ontogenic variations of ascrobic acid and phenathyl isothiocyanate concentration in watercress (Nasturtium officinale R.Br.) leaves. J. Agric. Food Chem., 2003, 51(18), 5504-5509.

[543]. Rose, P.; Faulkner, K.; Williamson, G.; Mithen, R. 7-Methylsulfinylheptyl and 8- methylsulfinyloctyl isothiocyanates from watercress are potent inducers of phase II enzymes. Carcinogenesis, 2000, 21(11), 1983-1988.

[544]. Conaway, C.C.; Jiao, D.; Chung, F.L. Inhibition of rat liver cytochrome P450 isozymes by isothiocyanate and their conjugates, a structure activity relationship study. Carcinogenesis, 1996, 17(11), 2423-2427.

[545]. Wallig, M.A.; Kingston, S.; Staack, R.; Jeffery, E.H. Induction of rat pancreatic glutathione -S- transferase and quinone reductase activities by a mixture of glucosinolate break down derivatives found in Brussel sprouts. Food Chem. Toxicol., 1998, 36, 365-373. 
[546]. Bianchet, M.A.; Foster, C.; Faig, M.; Talalay, P.; Amzel, L.M. Structure and mechanism of cytosolic quinone reductases. Biochem. Soc. Trans., 1999, 27(4), 610-615.

[547]. Asadi, M.S.; Mirvaghefei, A.R.; Nematollahi, M.A.; Banaee, M.; Ahmadi, K. Effects of Watercress (Nasturtium nasturtium) extract on selected immunological parameters of rainbow trout (Oncorhynchus mykiss). Open Vet. J., 2012, 2, 32-39.

[548]. Puri, A.; Saxena, R.; Saxena, R.P.; Saxena, K.C.; Srivastava, V.; Tandon, J.S. Immunostimulant activity of Nyctanthes arbor-tristis L. J. Ethnopharmacol., 1994, 42, 31-37.

[549]. Rani, C.; Chawla, S.; Mangal, M.; Mangal, A.K.; Kajla, S.; Dhawan, A.K. Nyctanthes arbor-tristis Linn. (Night Jasmine): A sacred ornamental plant with immense medicinal potentials. Indian J. Trad. Know., 2012, 11(3), 427-435.

[550]. Banerjee, A.; Poddar, A.; Ghanta, S.; Chakraborty, A.; Chattopadhyay, S. Nyctanthes arbor-tristis Linn. - Spectrum of its bioactivity potential. Planta Med., 2007, 73, SL 006.

[551]. Rathee, J. S.; Hassarajani, S.A.; Chattopadhyay, S. Antioxidant activity of Nyctanthes arbor-tristis leaf extract. Food Chem., 2007, 103( 4), 1350-1357.

[552]. Khan, Z.K.; Magnlani, A.; Shukla, P.K.; Puri, A.; Saxena, R.P.; Tandon, J. Immunomodulatory effect of plant extract and iridoid glycosides from Nyctanthes arbour-tristis against systemic candidiasis in mice. Int. J. Pharmacog., 1995, 33, 297-304.

[553]. Deb, A.; Barua, S.; Das, B. Pharmacological activities of Baheda (Terminalia bellerica): A review. J. Pharmacog. Phytochem., 2016, 5(1), 194-197.

[554]. Vaibhav, D.A.; Arun, K.; Wahi. Immunomodulatory effect of alcoholic extract of Terminalia chebula ripe fruits. $J$. Pharm. Sci. Res., 2010, 2(9), 539-544.

[555]. Ahn, M.J.; Kim, C.Y.; Lee, J.S.; Kim, T.G.; Kim, S.H.; Lee, C.K.; Lee, B.B.; Shin, C.G.; Huh, H.; Kim, J. Inhibition of HIV-1 integrase by galloyl glucoses from Terminalia chebula and flavonol glycoside gallates from Euphorbia pekinensis. Planta Med., 2002, 68, 454-457.

[556]. Kim, H.G.; Cho, H.G.; Jeong, E.Y.; Lim, J.H.; Lee, S.H. Growth-inhibiting activity of active component isolated from Terminalia chebula fruits against intestinal bacteria. J. Food Prot., 2006, 69, 2205-2209.

[557]. Rao, N.K.; Nammi, S. Antidiabetic and renoprotective effects of the chloroform extract of Terminalia chebula Retz. seeds in streptozotocin-induced diabetic rats. Complement. Alter. Med., 2006, 17, $1-6$.

[558]. Gandhi, N.M., Nair, C.K. Radiation protection by Terminalia chebula: some mechanistic aspects. Mol. Cell. Biochem., 2005, 277, 43-48.

[559]. Saleem, A.; Husheem, M.; Harkonen, P.; Pihlaja, K. Inhibition of cancer cell growth by crude extract and the phenolics of Terminalia chebula retz. Fruit. J. Ethnopharmacol., 2002, 81, 327-336.

[560]. Cheng, H.Y.; Lin, T.C.; Yu, K.H.; Yang, C.M.; Lin, C.C. Antioxidant and free radical scavenging activities of Terminalia chebula. Biol. Pharm. Bull., 2003, 26, 1331-1335.

[561]. Lee, H.S.; Won, N.H.; Kim, K.H.; Lee, H.; Jun, W.; Lee, K.W. Antioxidant effects of aqueous extract of Terminalia chebula in vivo and in vitro. Biol. Pharm. Bull., 2005, 28, 1639-1644.

[562]. Rubab, I.; Ali, S. Dried fruit extract of Terminalia chebula modulates the immune response in mice. Food Agri. Immunol., 2016, 27(1), 1-22.

[563]. Malekzadeha, F.; Ehsanifara, H.; Shahamatb, M.; Levinb, M.; Colwell, R.R. Antibacterial activity of black myrobalan (Terminalia chebula Retz) against Helicobacter pylori. Inter. J. Antimicro. Agents, 2001, 18(1), 85-88.

[564]. Pundareekaksha Rao, P. Ophthalmic uses of Boerhaavia diffusa L. (Punarnava): Review. Inter. J. Herbal Med., 2016; 4(2), 05-09.

[565]. Chude, M.A.; Orisakwe, O.J.; Afonne, O.J.; Gamaniel, K.S.; Vongtau, O.H.; Obi E. Hypoglycemic effect of the aqueous extract of Boerhavia diffusa leaves. Indian J. Pharmacol., 2001, 33, 215-216.

[566]. Hiruma-Lima, C.A.; Gracioso, J.S.; Bighetti, E.J.; Robineou L.G.; Brito, A.R.S. The juice of fresh leaves of Boerhaavia diffusaL.(Nyctaginaceae) markedly reduces pain in mice. J. Ethanopharmacol., 2000, 71, 261-274.

[567]. Pari, L.; Satheesh, A.M. Antidiabetic activity of Boerhaavia diffusa L.: effect on hepatic key enzymes in experimental diabetes. J. Ethnopharmacol., 2004, 91(1), 109-113.

[568]. Leslie, T. Erva Tostao In: The Healing Power of Rainforest Herbs, A guide to understanding and using herbal medicinals. Square one publishers, USA. 2005b, 272- 275.

[569]. Devi, K.M.; Jyothi, Y. Pharmacodynamic interaction of Boerhaavia diffusa with omeprazole in experimentally induced ulcers in rats. Indian J. Pharm. Biol. Res., 2015, 3(1), 56-63.

[570]. Mungantiwar, A.A.; Nair, A.M.; Shinde, U.A.; Dikshit, V.J.; Saraf, M.N.; Thakur, V.S.; Sainis, K.B. Studies on the immuno modulatory effects of Boerhaavia diffusa alkaloidal fraction. J Ethanopharmacol., 1999, 65, 125- 131.

[571]. Mehrotra, S. ; Mishra, K.P.; Maurya, R.; Srimal, R.C.; Singh, V.K. Immunomodulation by ethanolic extract of Boerhaavia diffusa roots. Int. Immunopharmacol., 2002, 7, 987-996.

[572]. Manu, K.A.; Kuttan, G. Immunomodulatory activities of Punarnavine, an alkaloid from Boerhaavia diffusa. Immunopharmacol. Immunotoxicol., 2009, 31(3), 377-387.

[573]. Olukoya, D.K.; Idika, N.; Odugbemi, T. Antibacterialv activity of some medicinalplants from Nigeria $J$. Ethanopharmacol., 1993, 39, 69-72.

[574]. Perumal Samy, R.; Ignacimuthu, S.; Raja, D.P. Ethanomedicinal plants from India. J. Ethanopharmacol., 1999, 66, 235-240.

[575]. Chandan, B.K.; Sharma, A.K.; Anand, K.K. Boerhaavia diffusia: a study of its hepatoprotective activity.J. Ethano pharmacol., 1991, 31, 299-307.

[576]. Rawat, A.K.; Mehrotra, S.; Tripathi, S.C.; Shome, U. Hepatoprotective activity of Boerhaavia diffusa.L.roots-a popular Indian ethanomedicine. J.Ethanopharmacol., 1997, 56, 61-62. 
[577]. Olaleye, M.T.; Rocha, J.B.T. Acetaminophen-induced liver damage in mice: effects of some medicinal plants on oxidative defense system. Exp. Toxicol. Pathol., 2008, 59, 319-327.

[578]. Olaleye, M.T.; Akinmoladun, A.C.; Ogunboye, A.A.; Akindahunsi, A.A. Antioxidant activity and hepatoprotective property of leaf extracts of Boerhaavia diffusa Linn against acetaminophen-induced liver damage in rats. Food Chem. Toxicol., 2010, 48(8-9), 2200-2205.

[579]. Nandi, R.; Ghosh, R. Phytochemical and Biological Importance of Boerhavia diffusa: A Plant of Ethnopharmacological knowledge. Int. J. Pharma. Sci. Res., 2016, 7(3), 134-143.

[580]. Alagawany, M.M.; Farag, M.R.; Dhama, K.; Abd El-Hack, M.E.; Tiwari, R.; Alam, G.M. Mechanisms and beneficial applications of resveratrol as feed additive in animal and poultry nutrition. A review. Int. J. Pharmacol., 2015b, 11(3), 213-221.

[581]. Cheeke, P.R.; Piacente, S.; Oleszek, W. Anti-inflammatory and anti-arthritic effects of yucca schidigera: A review. $J$. Inflamm. (Lond). 2006, 3, 6.

[582]. Ashour, E.A.; Alagawany, M.; Reda, F.M.; Abd El-Hack, M.E. Effect of supplementation of Yucca schidigera to growing rabbits diets on growth performance, carcass characteristics, serum biochemistry and liver oxidative status. Asian J. Anim. Vet. Adv., 2014, 9(11), 732-742.

[583]. Ettefagh, K.A.; Burns, J.T.; Junio, H.A.; Kaatz, G.W.; Cech, N.B. Goldenseal (Hydrastis canadensis L.) extracts synergistically enhance the antibacterial activity of berberine via efflux pump inhibition. Planta Med., 2011, 77(8), 835-840.

[584]. Scazzocchio, F.; Cometa, M.F.; Tomassini, L.; Palmery, M. Antibacterial activity of Hydrastis canadensis extract and its major isolated alkaloids. Planta Med., 2001, 67(6), 561-4.

[585]. Hwang, B.Y.; Roberts, S.K.; Chadwick, L.R.; Wu, C.D.; Kinghorn, A.D. Antimicrobial constituents from goldenseal (the Rhizomes of Hydrastis canadensis) against selected oral pathogens. Planta Med., 2003, 69(7), 623-627.

[586]. Chadwick, L.R.; Wu, C.D.; Kinghorn, A.D. Isolation of alkaloids from goldenseal (Hydrastis canadensis rhizomes) using pH-zone refining countercurrent chromatography. J. Liq. Chrom. Rel. Technol., 2001, 24, 2245-2453.

[587]. Gentry, E.J.; Jampani, H.B.; Keshavarz-Shokri, A.; Morton, M.D.; Velde, D.V.; Telikepalli, H.; Mitscher, L.A.; Shawar, R.; Humble, D.; Baker, W. Antitubercular natural products: berberine from the roots of commercial Hydrastis canadensis powder. Isolation of inactive 8-oxotetrahydrothalifendine, canadine, beta-hydrastine, and two new quinic acid esters, hycandinic acid esters-1 and -2. J. Nat. Prod., 1998, 61(10),1187-1193.

[588]. Abdel-Haq, H.; Cometa, M.F.; Palmery, M.; Leone, M.G.; Silvestrini, B.; Saso, L. Relaxant effects of Hydrastis canadensis L. and its major alkaloids on guinea pig isolated trachea. Pharmacol. Toxicol., 2000, 87(5), 218-222.

[589]. Roopashree, T.S.; Dang, R.; Shobha Rani, R.H.; Narendra, C. Antibacterial activity of antipsoriatic herbs: Cassia tora, Momordica charantia and Calendula officinalis. Int. J. Appl. Res. Nat. Prod., 2008, 1(3), 20-28.

[590]. Zitterl-Eglseer, K.; Reznicek, G.; Jurenitsch, J.; Novak, J.; Zitterl, W.; Franz, C. Morphogenetic variability of faradiol monoesters in marigold Calendula officinalis L. Phytochem. Anal., 2001, 12(3), 199.

[591]. Hamzawy, M.A.; El-Denshary, E.SM.; Hassan, N.S.; Mannaa, F.A.; Abdel-Wahhab, M.A. Dietary supplementation of Calendula officinalis counteracts the oxidative stress and liver damage resulted from aflatoxin. Inter. Scholar. Res. Notices, 2013, e538427.

[592]. Hamburger, M.; Adler, S.; Baumann, D.; Forg, A.; Weinreich, B. Preparative purification of the major antiinflammatory triterpenoid esters from Marigold (Calendula officinalis). Fitoterapia, 2003, 74(4), 328-338.

[593]. Miliauskas, G.; Venskutonis, P.R.; van Beek, T.A. Screening of radical scavenging activity of some medicinal and aromatic plant extracts. Food Chem., 2004, 85(2), 231-237.

[594]. Muley, B.P.; Khadabadi, S.S.; Banarase, N.B. Phytochemical constituents and pharmacological activities of Calendula officinalis Linn (Asteraceae): A review. Trop. J. Pharm. Res., 2009, 8(5), 455-465.

[595]. Silva, E.R.; Goncalves, E.S.; Aguiar, F.; Evencio, L.B.; Lyra, M.A.; Coelno, M.C.; Fraga, M.A.; Wanderloy, A.G. Toxicological studies on hydroalcoholic extract of Calendula officinalis L. Phytother. Res., 2007, 21, 332-336.

[596]. Sujatha, V.; Korde, J.P.; Rastogi, S.K.; Maini, S.; Ravikanth, K.; Rekhe, D.S. Amelioration of heat stress induced disturbances of the antioxidant defense system in broilers. J. Vet. Med. Anim. Health, 2010, 2(3), 18-28.

[597]. Maini, S.; Rastogi, S.K.; Korde, J.P.; Madan, A.K.; Shukla, S.K. Evaluation of oxidative stress and its amelioration through certain antioxidants in broilers during summer. J. Poult. Sci., 2007, 44, 339-347.

[598]. Calderwood, S.K.; Ciocca, D.R. Heat shock proteins: stress proteins with Janus-like properties in cancer. Int. J. Hyperthermia, 2008, 24, 31-39.

[599]. Vabulas, R.M.; Raychaudhuri, S.; Hayer-Hartl, M.; Hartl, F.U. Protein folding in the cytoplasm and the heat shock response. Cold Spr. Harbor Persp. Biol., 2010, 2(12), 1-18.

[600]. Wieten, L.; Broere, F.; van der Zee, R.; Koerkamp, E.K.; Wagenaar, J.; van Eden, W. Cell stress induced HSP are targets of regulatory T cells: A role for HSP inducing compounds as anti-inflammatory immuno-modulators? FEBS Letters, 2007, 58, 3716-3722.

[601]. Lumbera, W.M.L.; dela Cruz, J.; Yang, S.; Hwang, S.G. Heat Shock protein augmentation of Angelica gigas Nakai root hot water extract on adipogenic differentiation in Murine 3T3-L1 Preadipocytes. Asian-Australas J. Anim. Sci., 2016, 29(3), 419-427.

[602]. Park, S.J.; Jung, J.M.; Lee, H.E.; Lee, Y.W.; Kim, D.H. The memory ameliorating effects of INM-76, an ethanolic extract of Angelica gigas, against scopolamine- or A $\beta$ (1-42)- induced cognitive dysfunction in mice. $J$. Ethnopharmacol., 2012, 143(2), 611-620.

[603]. Kato, K.; Ito, H.; Kamei, K.; Iwamoto, I. Stimulation of the stress-induced expression of stress proteins by curcumin in cultured cells and in rat tissues in vivo. Cell Stress Chaperones, 1998, 3, 152-160. 
[604]. Funk, J.L.; Frye, J.B.; Oyarzo, J.N.; Kuscuoglu, N.; Wilson, J.; McCaffrey, G.; Stafford, G.; Chen, G.; Lantz, R.C.; Jolad, S.D.; Solyom, A.M.; Kiela, P.R.; Timmermann, B.N. Efficacy and mechanism of action of turmeric supplements in the treatment of experimental arthritis. Arthrit. Rheum., 2006, 54, 3452-3464.

[605]. Swathi, B.; Gupta, P.S.P.; Nagalakshmi, D.; Reddy, A.R.; Raju, M.V.L.N. Immunomodulatory and cortisol sparing effect of tulsi (Ocimum sanctum) in heat stressed broilers. Tamilnadu J. Vet. Anim. Sc., 2012, 9(1), 23 - 28.

[606]. Yan, D.; Saito, K.; Ohmi, Y.; Fujie, N.; Ohtsuka, K. Paeoniflorin, a novel heat shock protein-inducing compound. Cell Stress Chaperones, 2004, 9, 378-389.

[607]. Westerheide, S.D.; Bosman, J.D.; Mbadugha, B.N.; Kawahara, T.L.; Matsumoto, G.; Kim, S.; Gu, W.; Devlin, J.P.; Silverman, R.B.; Morimoto, R.I. Celastrols as inducers of the heat shock response and cytoprotection. J. Biol. Chem., 2004, 279, 56053-56060.

[608]. Yoon, K.Y.; Kim, K.J.; Lee, H.H.; Hong, H.D.; Lee, B.Y. Protective Effect of Ginseng and Several Medicinal Herbs on Heat Stress by IFIT1 and IFIT2 Gene Expression. FASEB J., 2008, 22, 1108.5.

[609]. Al-Ramadan, M.M.; Alamer, M. Arab Gulf J. Sci. Res., 2010, 28(4), 224-231.

[610]. Chen, Y.Y.; Xu, G.Z.; Zhang, K.C. Effects of Chinese Herbal Drugs as Additives on yield performance and physiological parameters of heat stress cow. J. Dairy Sci., 2010, 1, 39-42.

[611]. Guo, K.J.; Xu, S.F.; Yin, P.; Wang, W.; Song, X.Z.; Liu, F.H.; Xu, J.Q.; Zoccarato, I. Active components of common traditional Chinese medicine decoctions have antioxidant functions. J. Anim. Sci., 2011, 89(10), 3107-3115.

[612]. Wilmsen, P.K.; Spada, D.S.; Salvador, M. Antioxidant activity of the flavonoid hesperidin in chemical and biological systems. J. Agric. Food Chem., 2005, 53(12), 4757-61.

[613]. Talbott, S.M.; Talbott, J.A.; Pugh, M. Effect of Magnolia officinalis and Phellodendron amurense (Relora $\left.{ }^{\circledR}\right)$ on cortisol and psychological mood state in moderately stressed subjects. J. Int. Soc. Sports Nutr., 2013, 10(1), 37.

[614]. Pandey, M.; Debnath, M.; Gupta, S.; Chikara, S.K. Phytomedicine: An ancient approach turning into future potential source of therapeutics. J. Pharmacog. Phytother., 2011, 3(3), 27-37.

[615]. Priyadarshini, M.; Manissery, J.K.; Mohan, C.V.; Keshavanath, P. Effect of ImmuPlus on growth and inflammatory response to Fruend's complete adjuvant in common carp, Cyprinus carpio (L.). Turkish J. Fisheries Aquatic Sci., 2012, 12, 291-299.

[616]. Okonkwo, C.; Oladele, O.; Nwiyi, P. The pattern of immunomodulation of ImmuPlus on the Infectious Bursal Disease (IBD) antibody of vaccinated broiler chickens. J. Vet. Adv., 2015, 5(1), 808-813.

[617]. Jena, G.B.; Kumar, V.S.; Nemmani, C.L.; Kaul, P.; Ramarao, P. Protective effect of a polyherbal formulation (Immu21) against cyclophosphamide-induced mutagenicity in mice. Phytother. Res., 2003, 17(4), 306-310.

[618]. Chatterjee S. Effect of immu-21, a herbal formulation on granulocytemacrophage colony stimulating factors, macrophage maturation and splenic plaque forming cells in experimental animals. Ind. J. Pharmacol., 2001, 33, 442444.

[619]. Silva, C.P.; Schwartz, F.F. The use of neem oil (Azadirachta indica a. juss) for the treatment of bovine udder ulcer. Oikos Agroecologia, 2003, 1-4.

[620]. Kumar, K.M.; Ramaiah, S. Pharmacological importance of Echinacea Purpurea. Int. J. Pharma Bio Sci., 2011, 2(4), 304-314.

[621]. Ngcobo, M.; Gqaleni, N.; Ndlovu, V.; Serumula, M.; Sibiya, N. Immunomodulatory effects of Umakhonya ${ }^{\circledR}$ : A South African commercial traditional immune booster. S. Afr. J. Bot., 2016, 102, 26-32.

[622]. Chu, W.K.; Cheung, S.C.; Lau, R.A.; Benzie, I.F.; Wachtel-Galor, S. Herbal medicine: biomolecular and clinical aspects. CRC Press, Boca Raton (FL). 2011.

[623]. Latheef, S.K.; Dhama, K.; Wani, M.Y.; Samad, H.A.; Tiwari, R.; Singh, S.D. Ameliorative effects of Withania somnifera, Azadirachta indica, Tinospora cordifolia and E Care Se herbal preparations on chicken infectious anaemia induced haematological changes in chicks and their live body weights. South Asian J. Exp. Biol., 2013, 3(4), $172-182$.

[624]. Latheef, S.K.; Dhama, K.; Samad, H.A.; Wani, M.Y.; Kumar M.A.; Palanivelu, et al. Immunomodulatory and prophylactic efficacy of herbal extracts against experimentally induced chicken infectious anaemia in chicks: assessing the viral load and cell mediated immunity. Virus Dis., 2017, doi:10.1007/s13337-016-0355-3.

[625]. Badar, V.A.; Thawanib, V.R.; Wakodec, P.T.; et al. Efficacy of Tinospora cordifolia in allergic rhinitis. J. Ethnopharmacol., 2005, 96, 445-449.

[626]. Krishan, G.; Shukla, S.K.; Bhatt, P.; Kumar, R.; Tiwari, R.; Malik, Y.S.; Dhama, K. Immunomodulatory and protective effects of a polyherbal formulation (Immon) against infectious anemia virus infection in broiler. Int. J. Pharmacol., 2015, 11(5), 470-476.

[627]. Bhatt, V.D.; Shah, T.M.; Nauriyal, D.S.; Kunjadia, A.P.; Joshi, C.G. Evaluation of a topical herbal drug for its invivo immunomodulatory effect on cytokines production and antibacterial activity in bovine subclinical mastitis. Ayu., 2014, 35(2), 198-205.

[628]. Kim, J.J.; Choi, J.; Lee, M.K.; Kang, K.Y.; Paik, M.J.; Jo, S.K.; Jung, U.; Park, H.R.; Yee, S.T. Immunomodulatory and antidiabetic effects of a new herbal preparation (hemoHIM) on streptozotocin-induced diabetic mice. Evid. Based Complement. Alternat Med., 2014, e461685.

[629]. Ngcobo, M.; Gqaleni, N.; Ndlovu, V.; Serumula, M.; Sibiya, N. Immunomodulatory effects of Umakhonya ${ }^{\circledR}$ : A South African commercial traditional immune booster. S. Afr. J. Bot., 2016, 102, 26-32.

[630]. Singh, H.P.; Singh, B.P.; Gulia, S.K. Research and application imperatives for the sustainable production of phytomedicines. International Symposium on Medicinal and Nutraceutical Plants, Macon, Georgia, USA. 2007.

[631]. Jia, L.; Zhao, Y. Current evaluation of the millenium phytomedicine - Ginseng (I): Etymology, Pharmacognosy, Phytochemistry, Market and regulations. Curr. Med. Chem., 2009, 16(19), 2475-2484.

[632]. Kaufman, P.B.; Cseke, L.J.; Warber, S.; Duke, J.A.; Brielmann, H.L. Natural Products from Plants. CRC Press, Boca Raton, FL, 1999. 
[633]. Wink, M. Introduction: biochemistry, role and biotechnology of secondary products. In M Wink, ed, Biochemistry of Secondary Product Metabolism. CRC Press, Boca Raton, FL, 1999, 1-16

[634]. Shaw D. Toxicological risks of Chinese herbs. Planta Med. 2010, 76(17), 2012-8.

[635]. Ansari, S.H.; Islam, F.; Sameem, M. Influence of nanotechnology on herbal drugs: A Review. J. Adv. Pharm. Tech. Res., 2012, 3(3), 142-146.

[636]. Perumal, S.R.; Gopalakrishnakone, P. Current status of herbal and their future perspectives. Nature Preceding, 2007, hdl:10101/npre.2007.1176.1: Posted 28 Sept. 